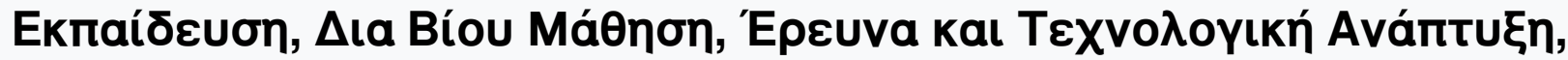 Katvotopía Kal Oıкоvopía
}

Tóp. 1 (2016)

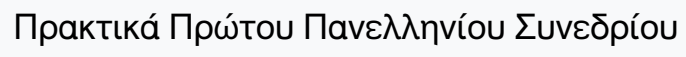

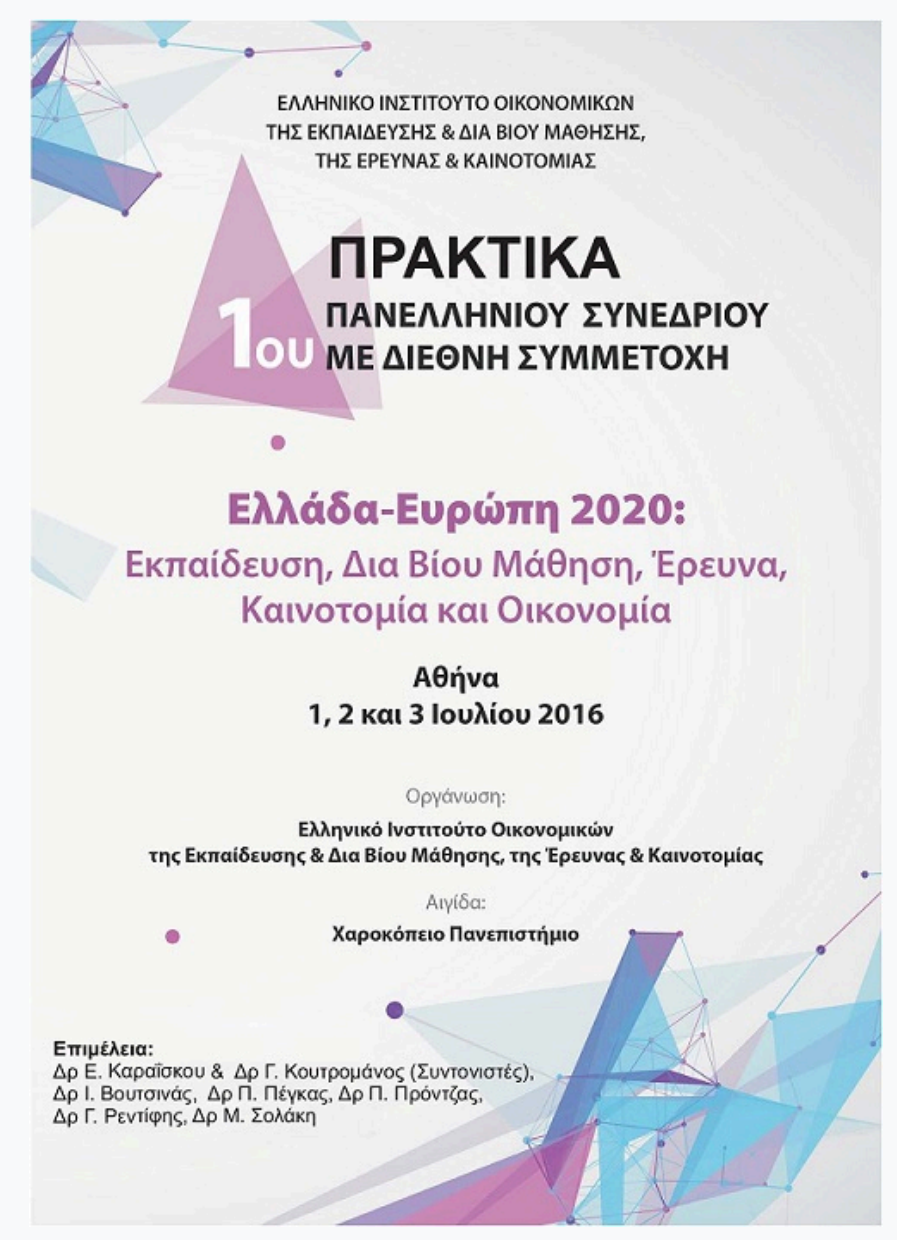

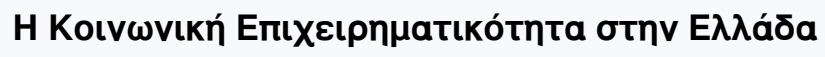
kaı Eupẃrn tou 2020

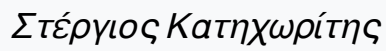

doi: $\underline{10.12681 / \text { elrie. } 798}$ 


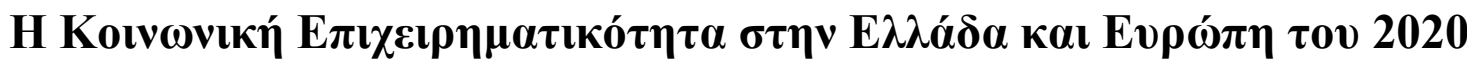

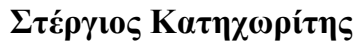

stergios.katichoritis@gmail.com

\begin{abstract}
The study investigates the role of Social Economy and Entrepreneurship and creating innovative conditions in the production process, technology and promoting cooperativity. Particularly, approaching cultural figures in Greece and the relationship with the structure of the current education system, since the values and principles to new sustainability cultures dependent learning. It approaches the participation of social entrepreneurship in a new sustainable economy and in particular relations with the evolution of the association in Greek rural economy. Finally, it shows the necessity of establishing social enterprises in Greece, hoping that it will help to promote social cohesion, the sustainability of the natural environment and improve the functioning of cities.
\end{abstract}

Keywords: Social, Economy, Entrepreneurship, Education

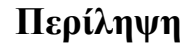

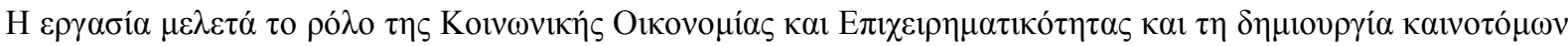

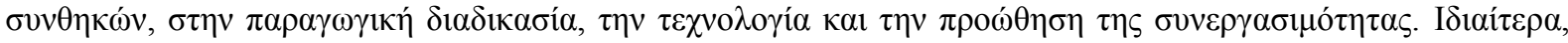

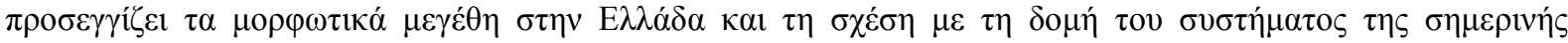

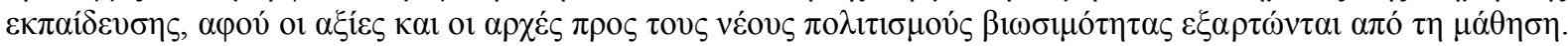

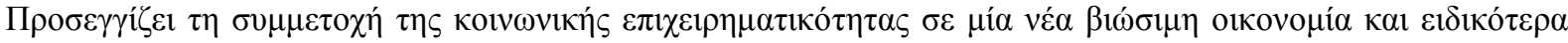

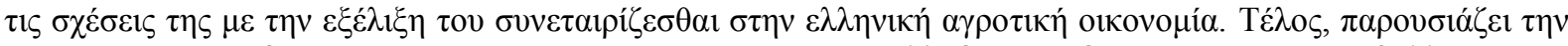

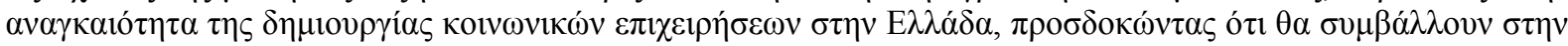

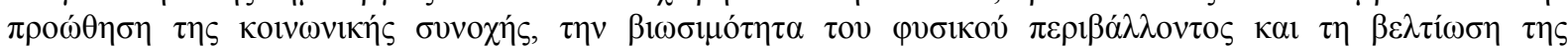
$\lambda \varepsilon i \tau o v p \gamma i ́ a s \tau \omega v \pi$ $\tau \dot{\lambda} \varepsilon \omega v$.

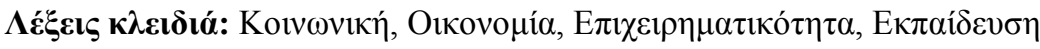

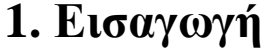

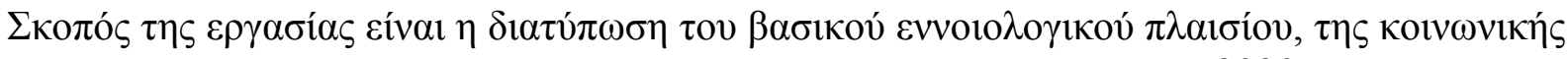

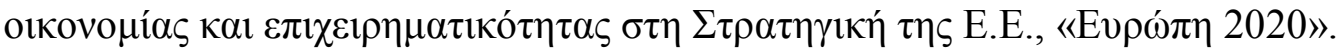

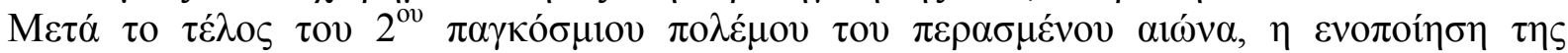

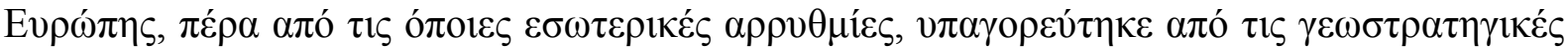

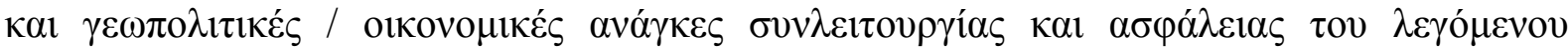

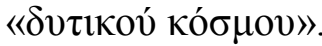

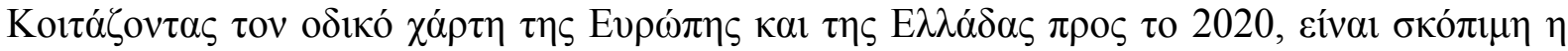

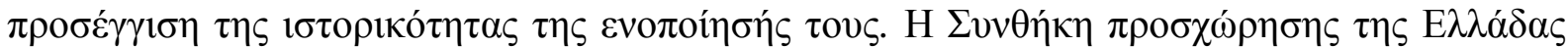

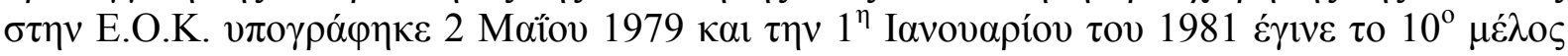

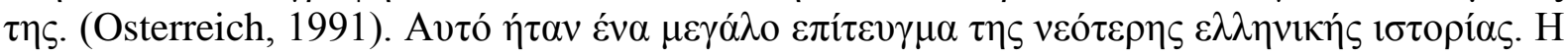

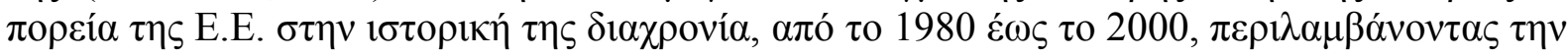

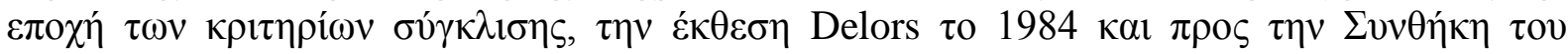

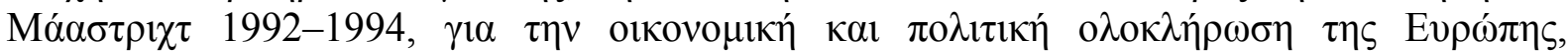

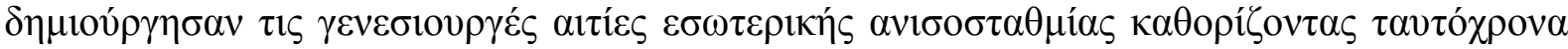

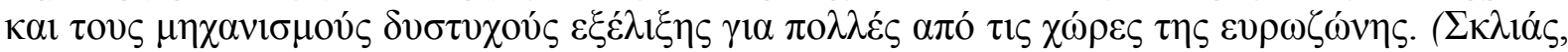

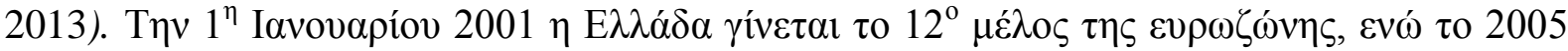

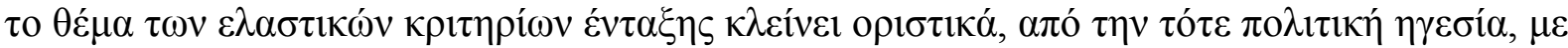

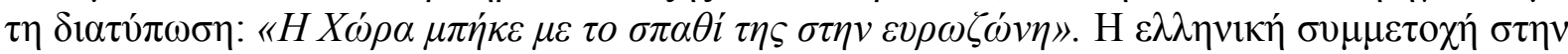




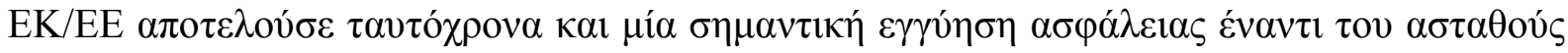

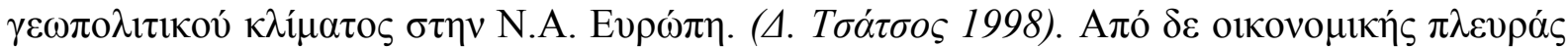

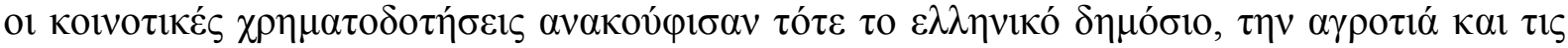

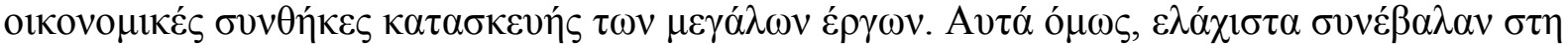

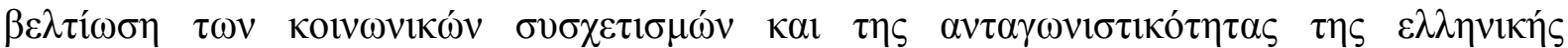

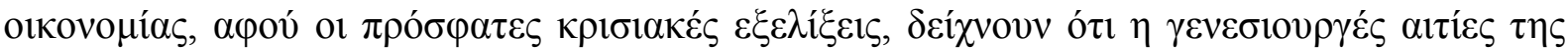

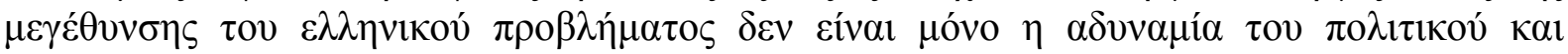

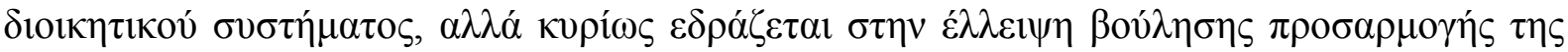

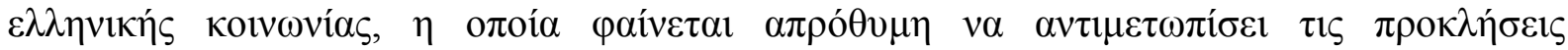

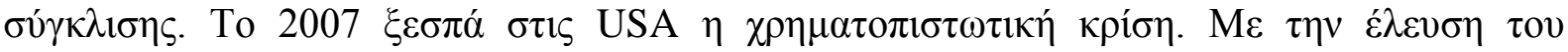

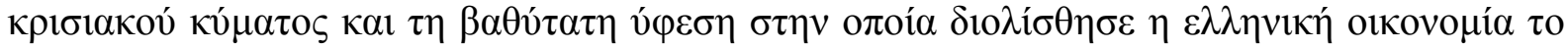

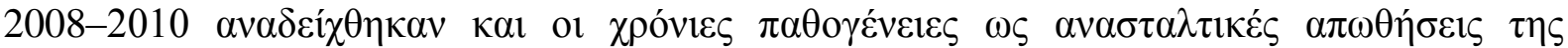

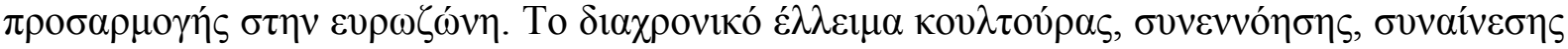

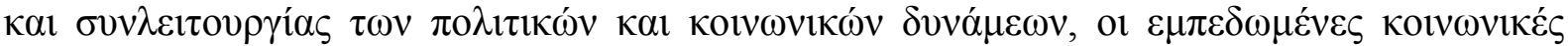

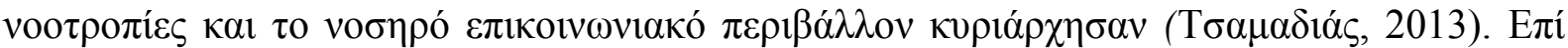

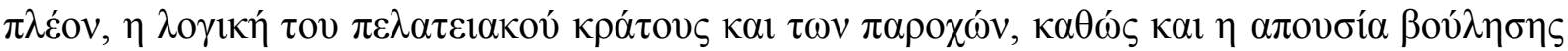

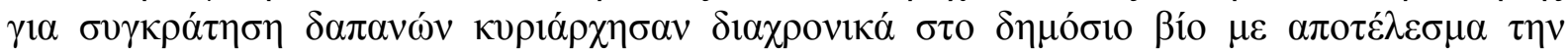

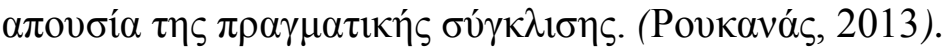

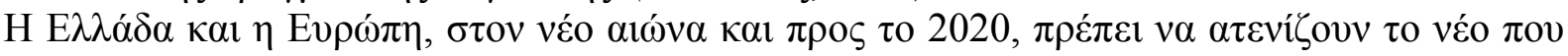

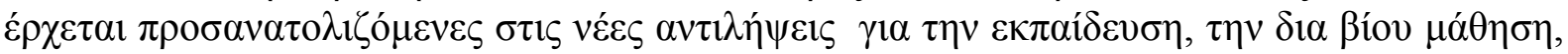

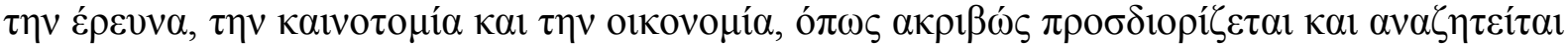

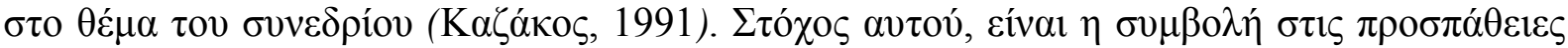

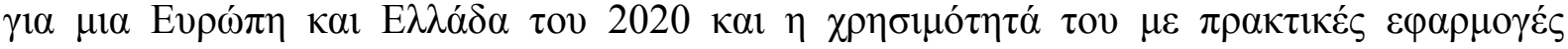

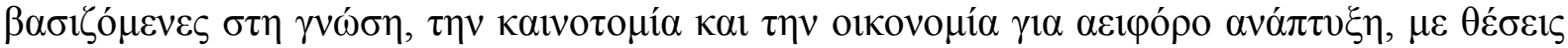

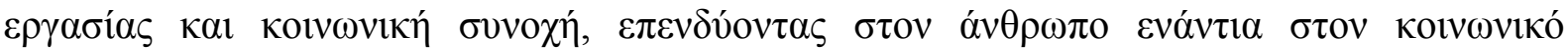

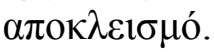

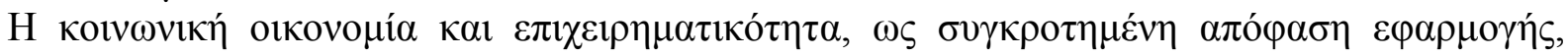

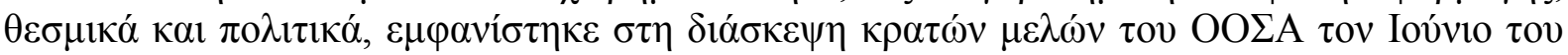

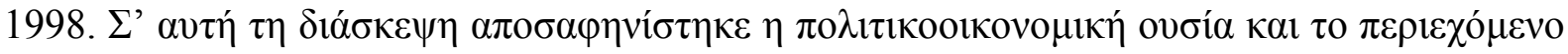

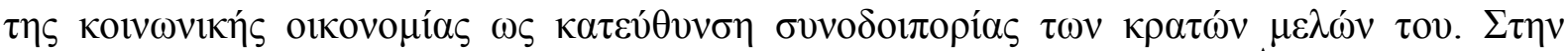

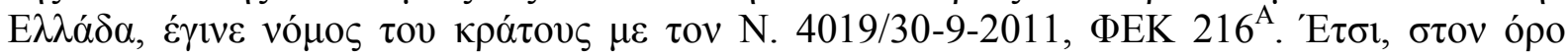

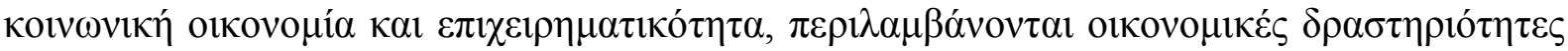

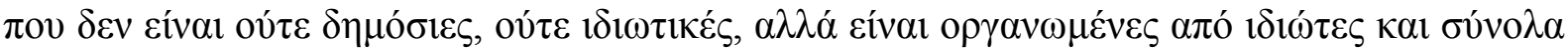

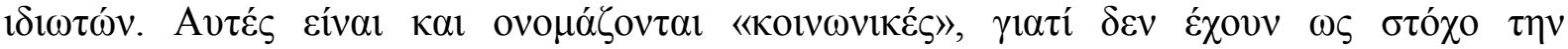

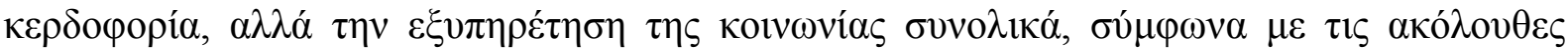

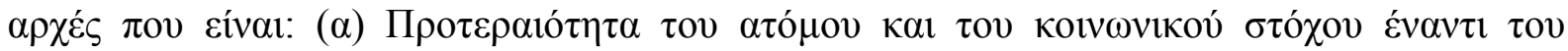

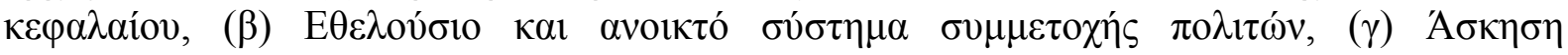

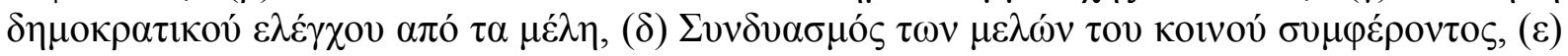

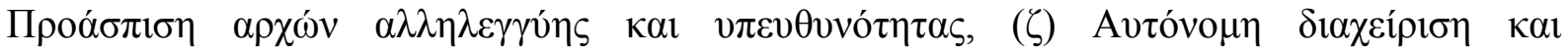

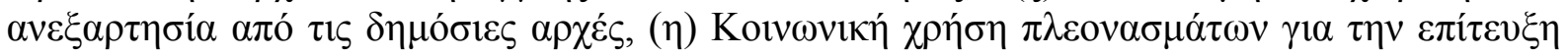

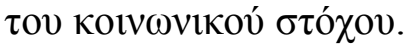

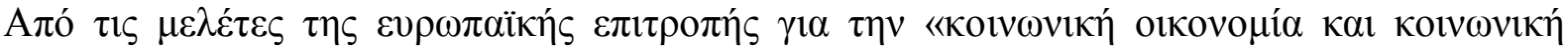

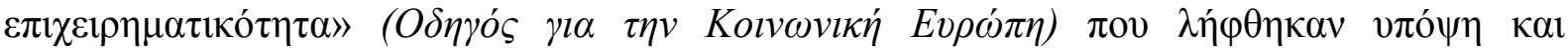

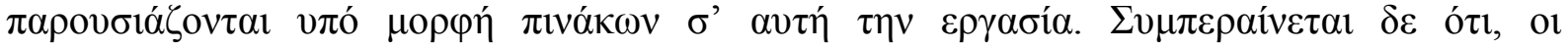

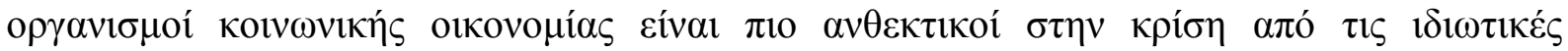

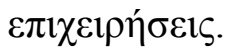

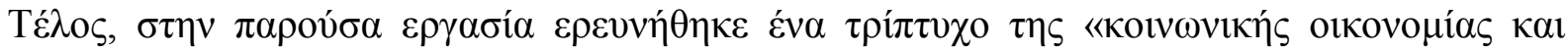

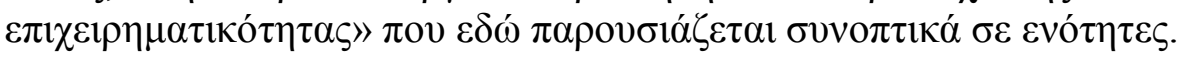




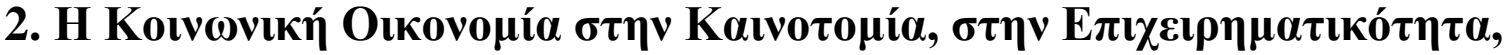

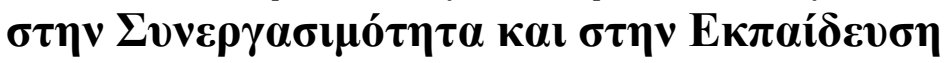

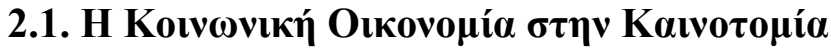

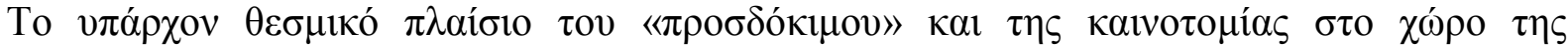

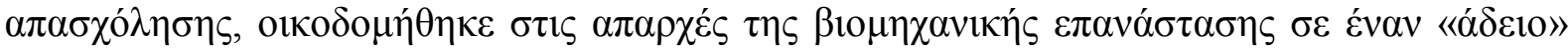

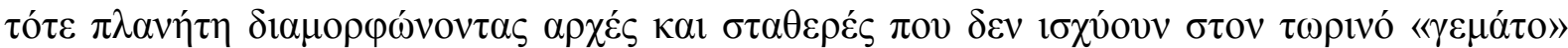

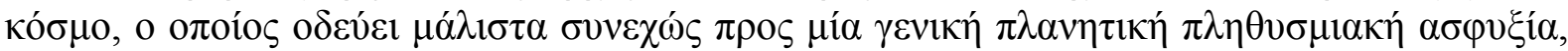

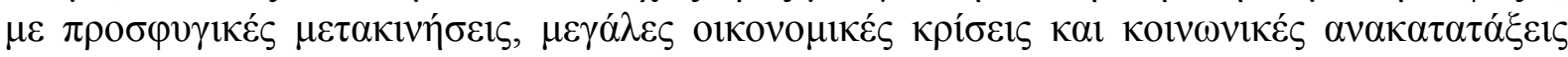

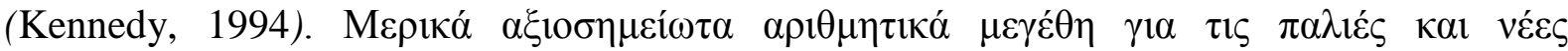

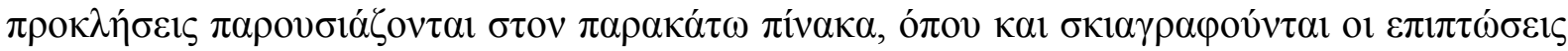

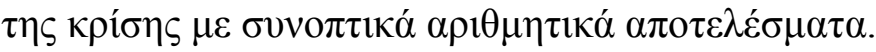

Пívakas 1

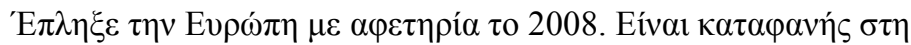

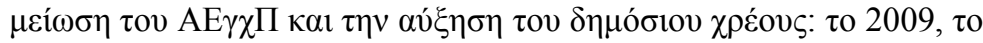

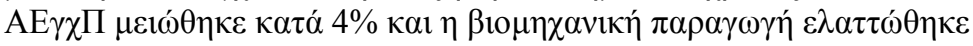

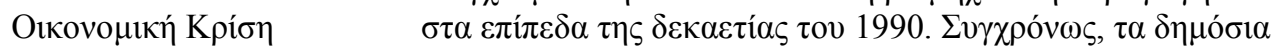

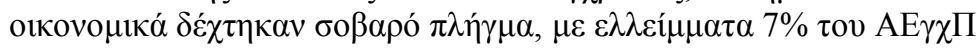

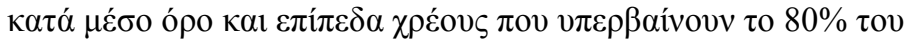
AE $\gamma \chi \Pi$.

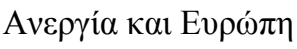

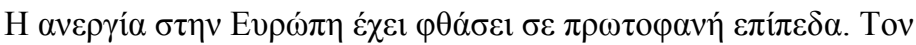

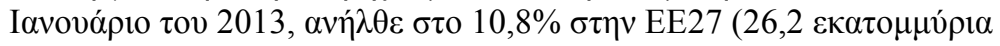

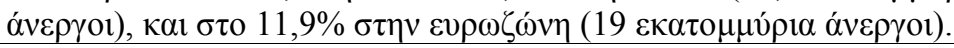

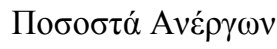

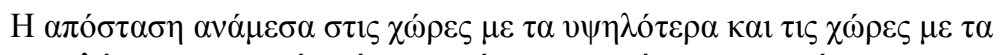

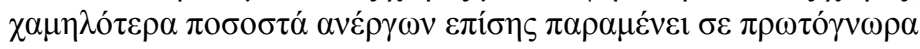

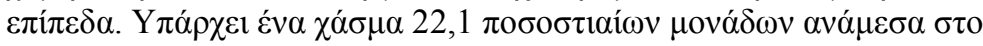

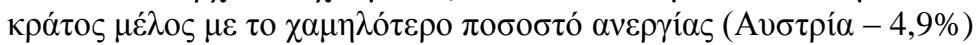

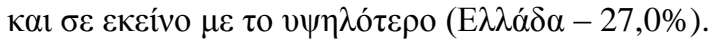

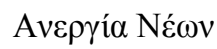

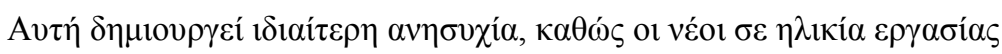

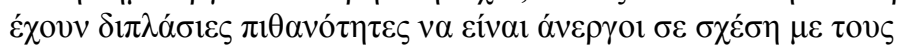

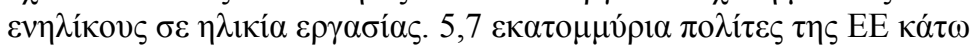

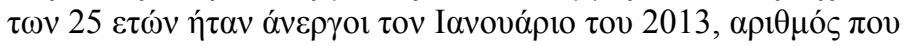

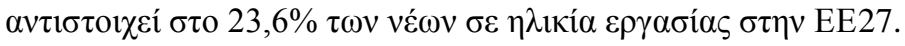

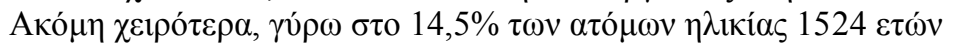

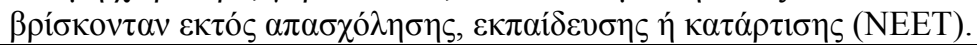

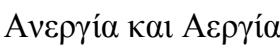

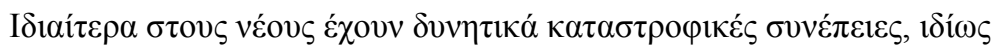

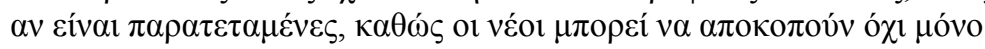

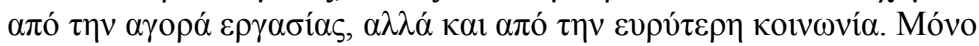

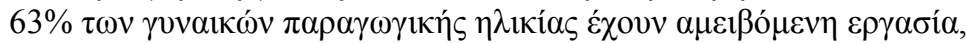

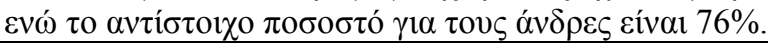

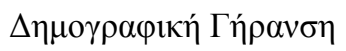

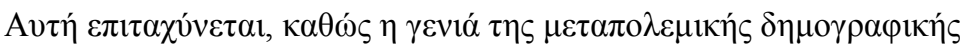

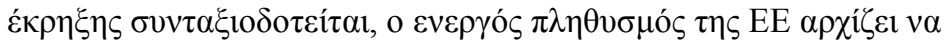

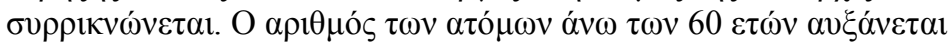

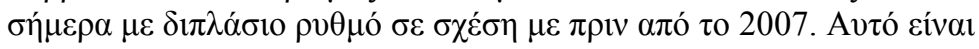

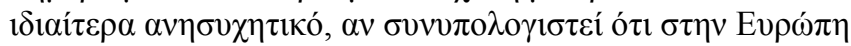
$\alpha \pi \alpha \sigma \chi 0 \lambda \varepsilon i ́ \tau \alpha 1 ~ \mu o ́ v o ~ \tau o ~ 46 \% ~ \tau \omega v ~ \varepsilon \rho \gamma \alpha \zeta o \mu \varepsilon ́ v \omega v ~ \mu \varepsilon \gamma \alpha \lambda v ́ \tau \varepsilon \rho \eta \varsigma ~ \eta \lambda ı i_{\alpha \varsigma}$

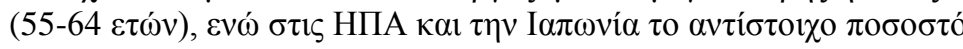

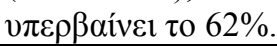

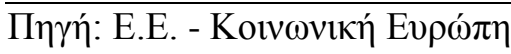

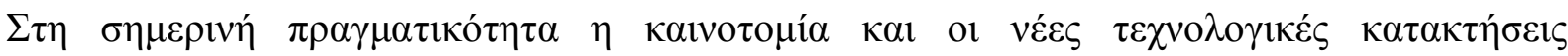

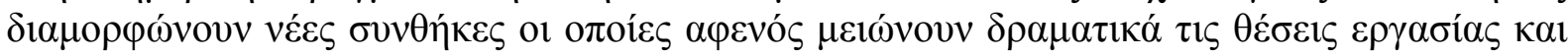

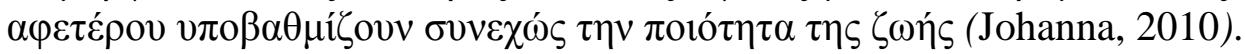




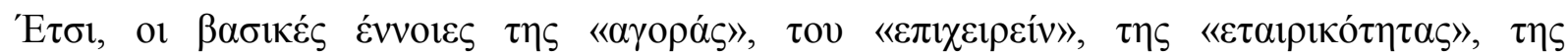

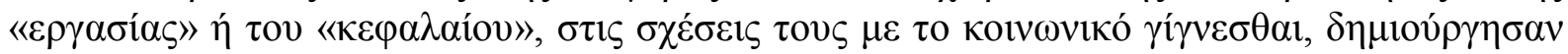

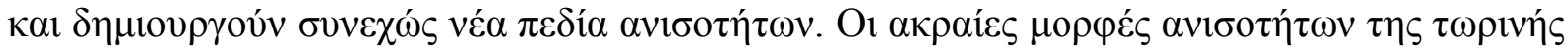

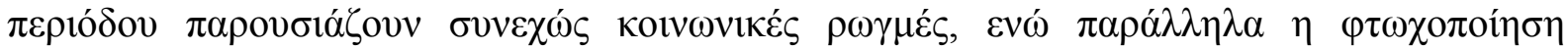

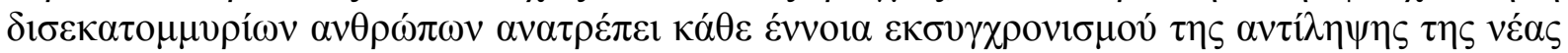

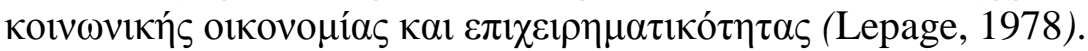

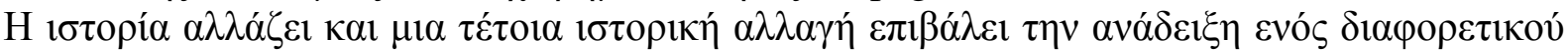

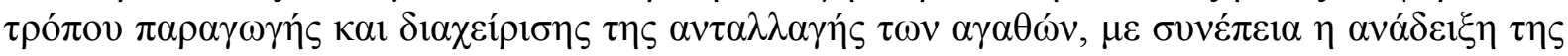

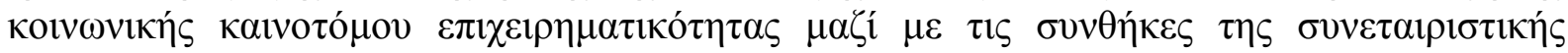

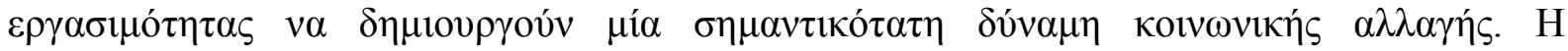

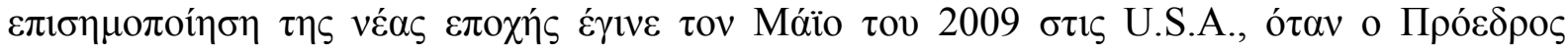

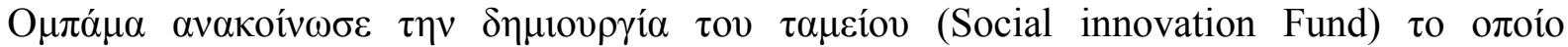

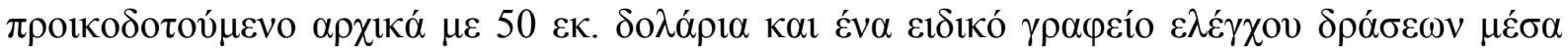

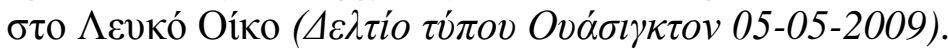

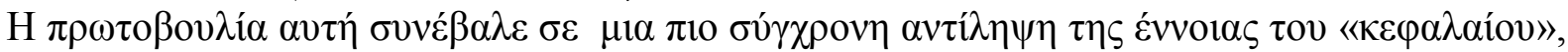

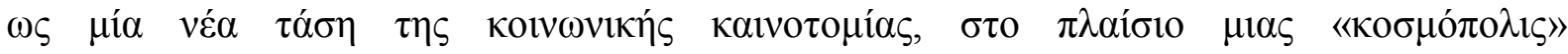

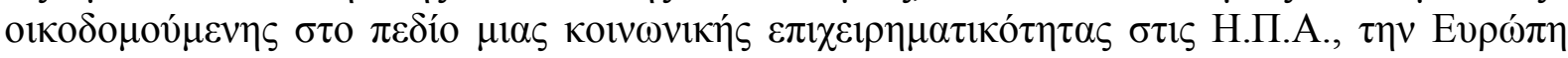

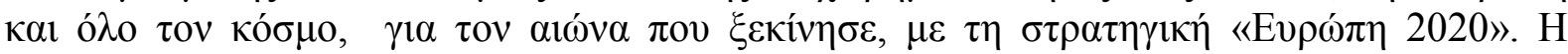

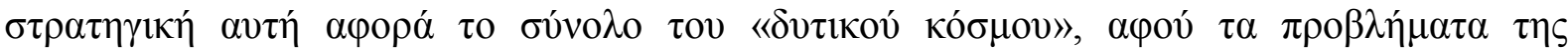

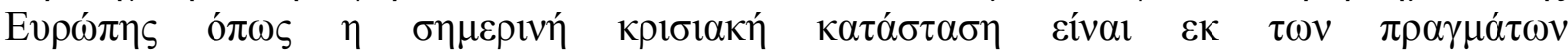

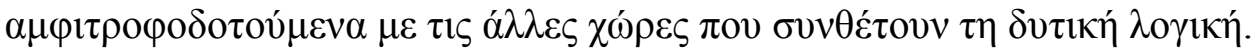

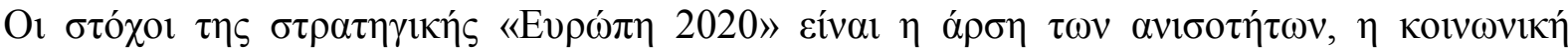

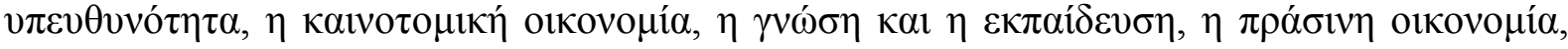

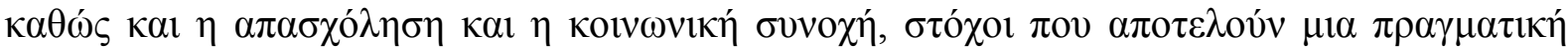

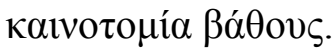

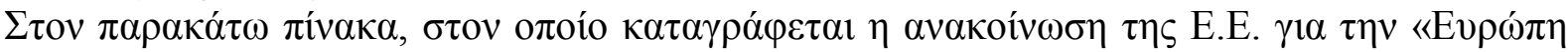

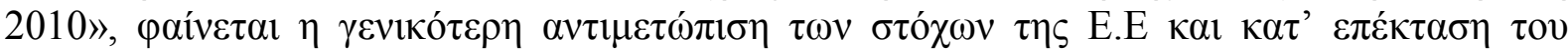

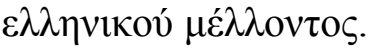

Пívakas 2:

\begin{tabular}{|c|c|}
\hline 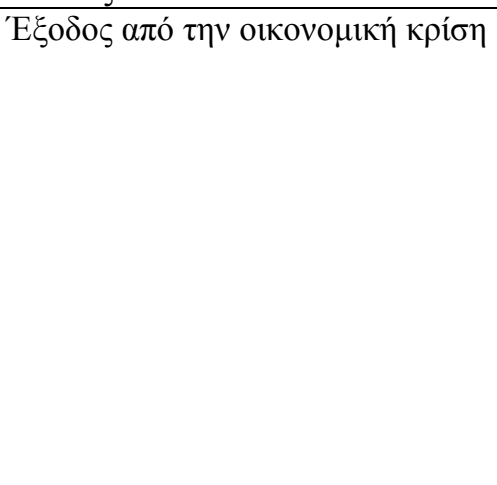 & 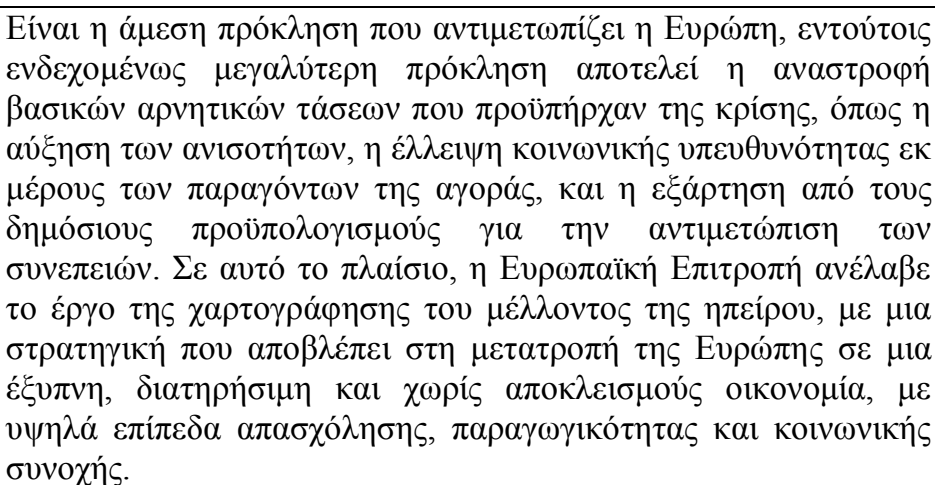 \\
\hline$\Sigma \tau \rho \alpha \tau \eta \gamma(\kappa \eta ́ ~ « E v \rho \omega ́ \pi \eta ~ 2020 »$ & 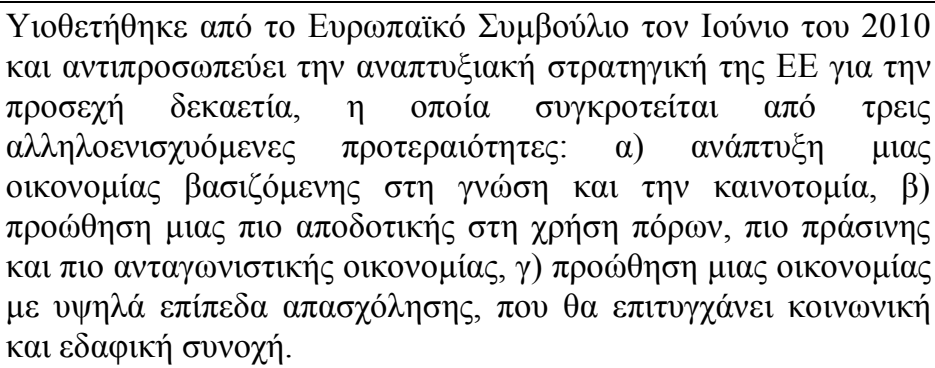 \\
\hline 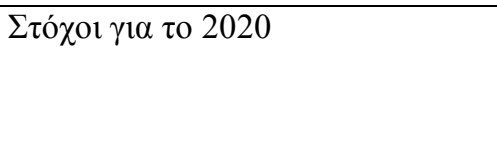 & 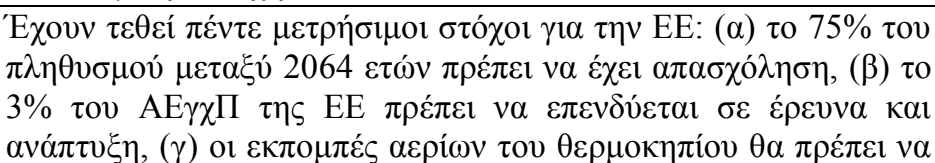 \\
\hline
\end{tabular}




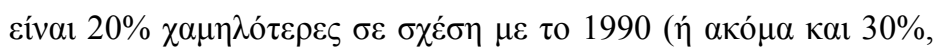

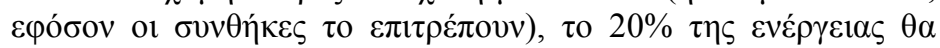

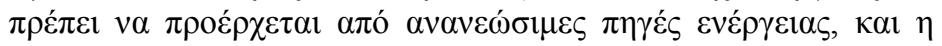

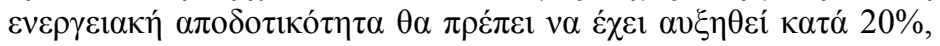

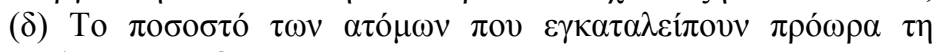

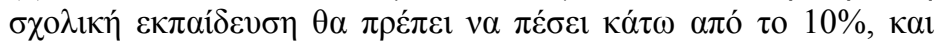

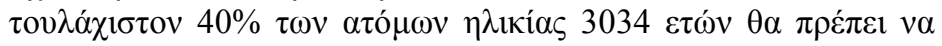

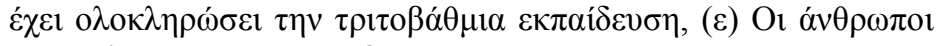

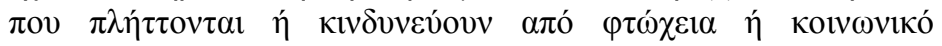

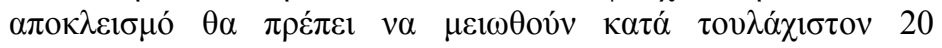
$\varepsilon \kappa \alpha \tau о \mu \mu v ́ \rho t \alpha$.

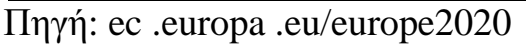

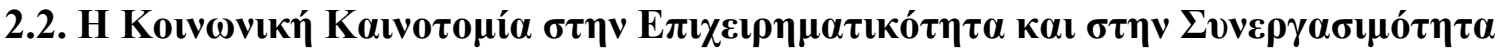

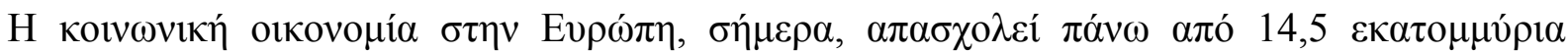

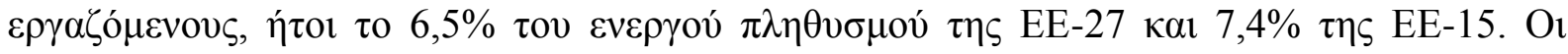

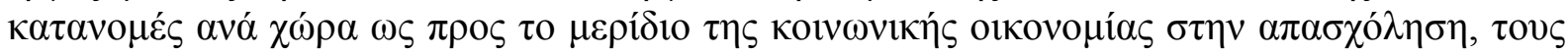

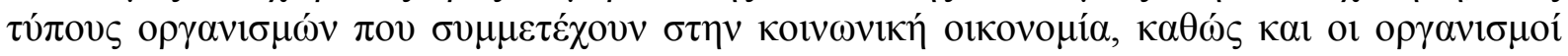

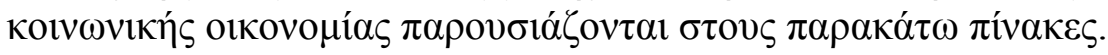

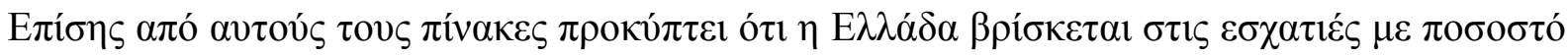
$2 \%-5 \%$. 


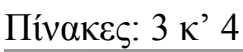

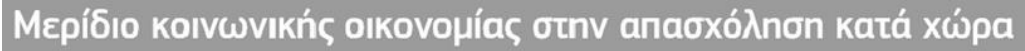

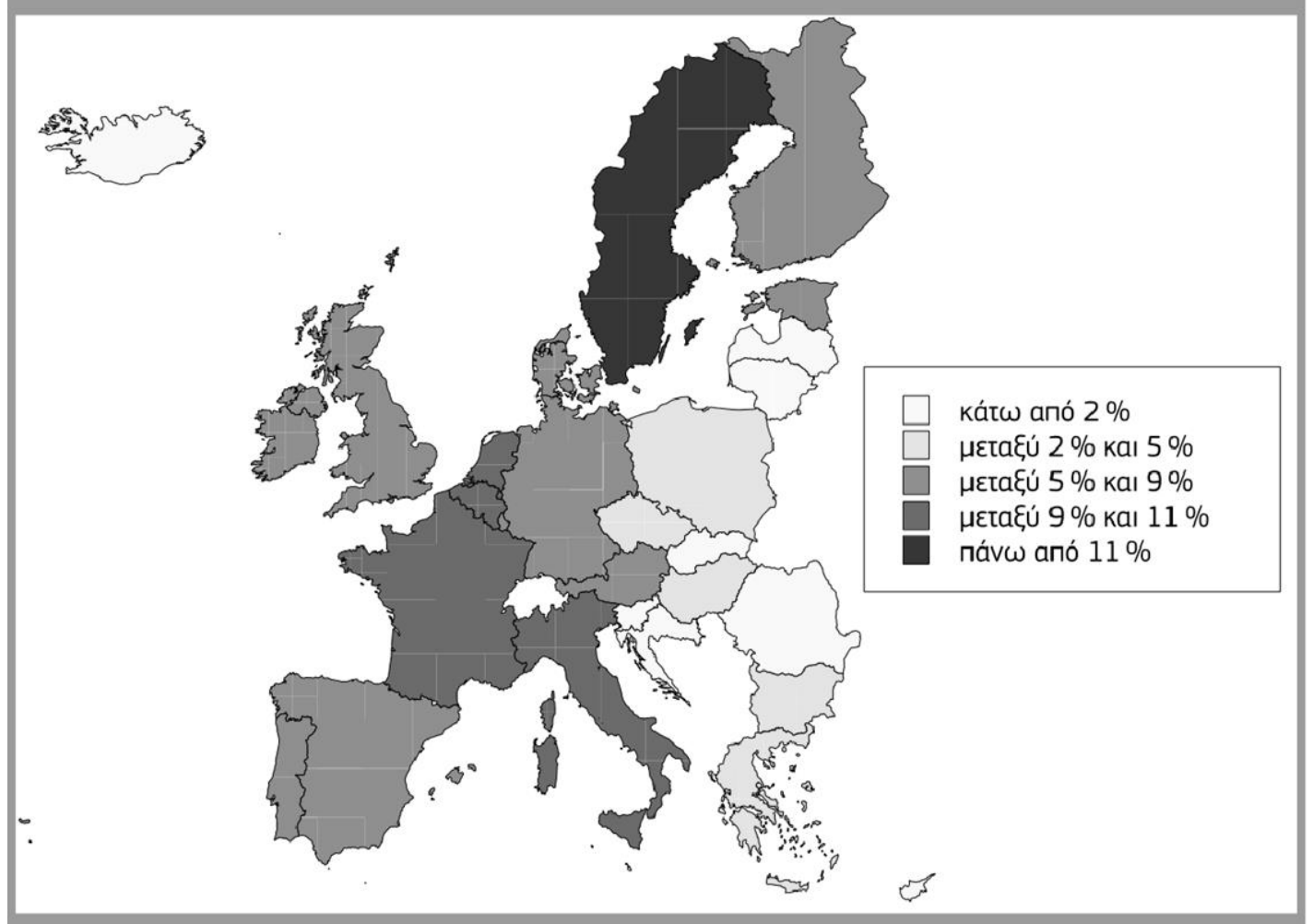

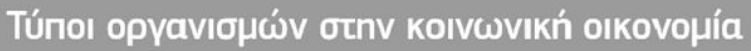

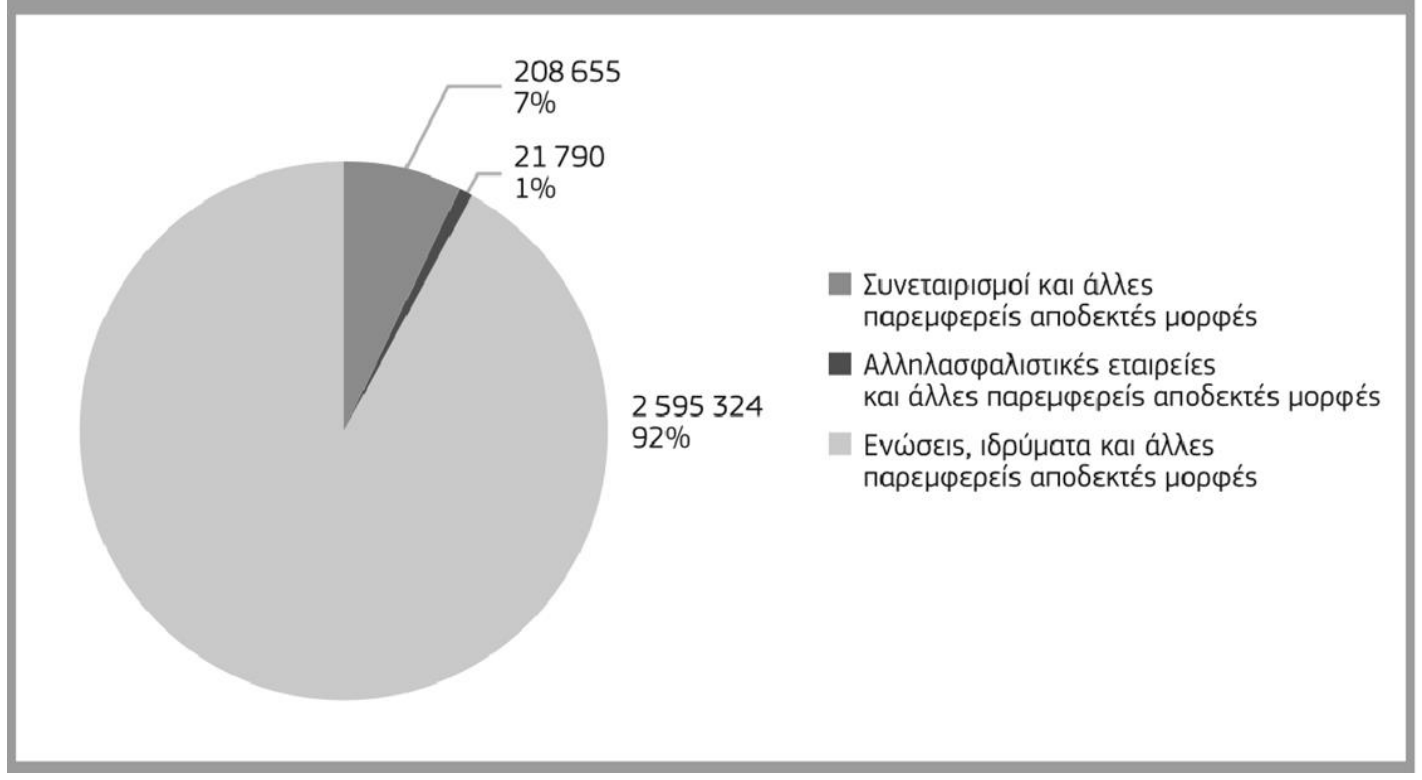


Пívakas 5

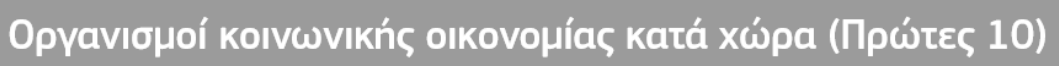

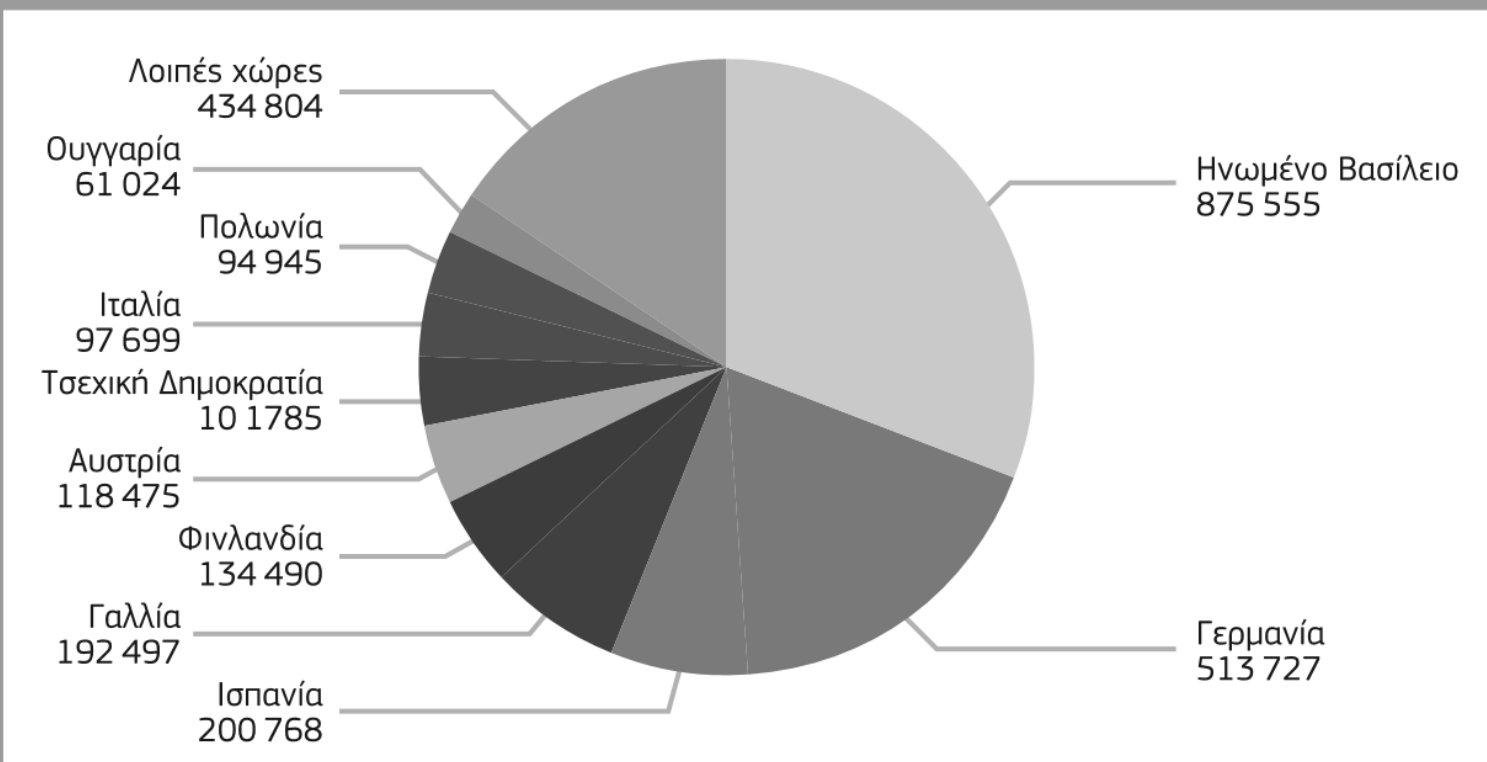

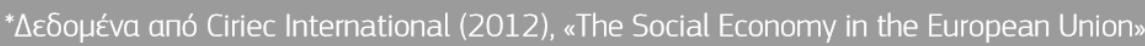

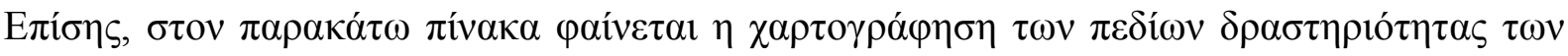

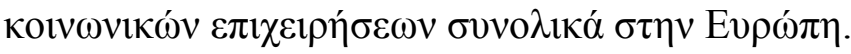

Пívakas 6

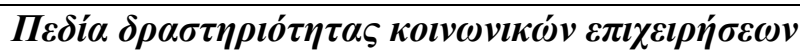

\begin{tabular}{|c|c|}
\hline 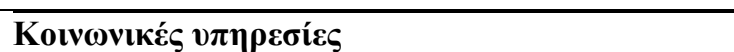 & $16,70 \%$ \\
\hline 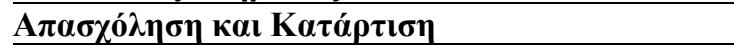 & $14,88 \%$ \\
\hline$\Pi \varepsilon \rho \iota \beta \dot{\alpha} \lambda \lambda о \mathrm{ov}$ & $14,52 \%$ \\
\hline 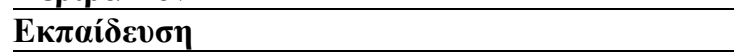 & $14,52 \%$ \\
\hline 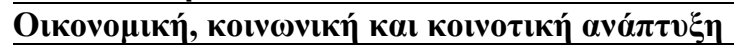 & $14,34 \%$ \\
\hline 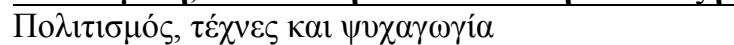 & $7,08 \%$ \\
\hline Yүzía & $6,90 \%$ \\
\hline$\sum \tau \dot{\varepsilon} \gamma \alpha \sigma \eta$ & $2,72 \%$ \\
\hline 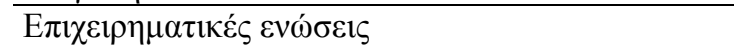 & $2,00 \%$ \\
\hline 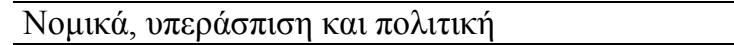 & $1,63 \%$ \\
\hline \multirow[t]{2}{*}{$\Lambda \mathrm{ol} \pi \dot{\alpha}$} & $4,72 \%$ \\
\hline & $100 \%$ \\
\hline
\end{tabular}

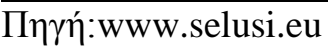

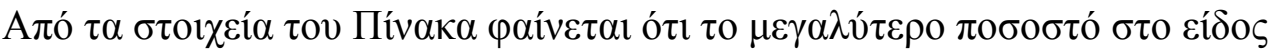

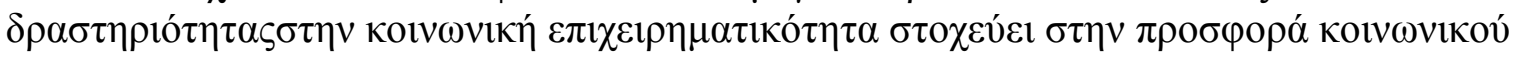

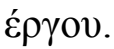

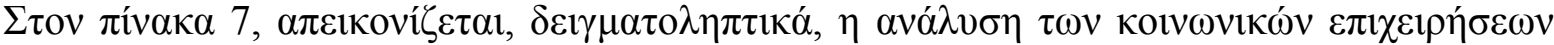

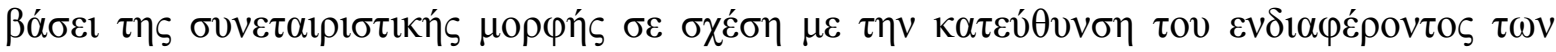

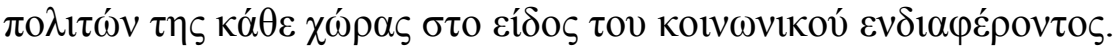


Пívakas 7

\begin{tabular}{|c|c|c|c|}
\hline 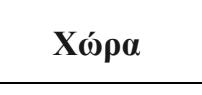 & 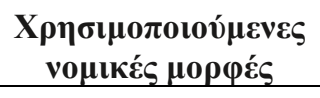 & 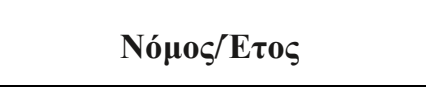 & 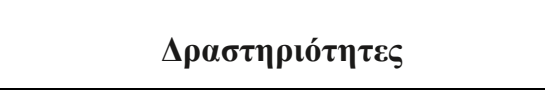 \\
\hline 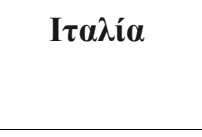 & 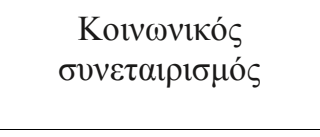 & $381 / 1991$ & 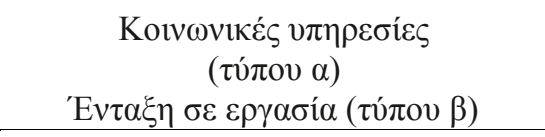 \\
\hline 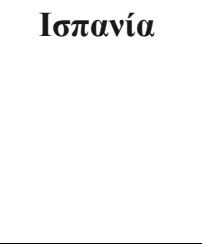 & 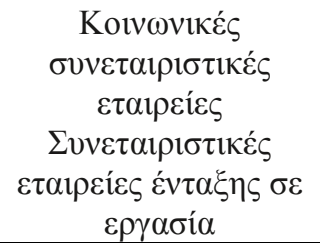 & 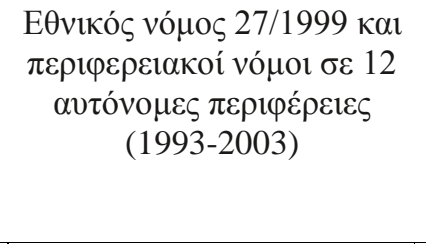 & 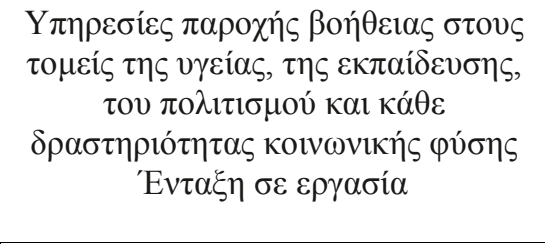 \\
\hline$\Gamma \alpha \lambda \lambda i ́ \alpha$ & 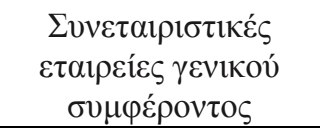 & 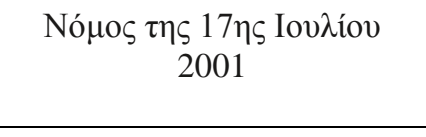 & 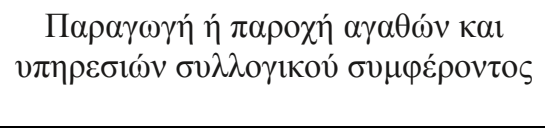 \\
\hline 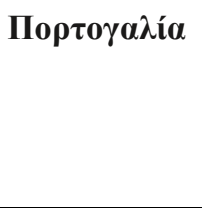 & 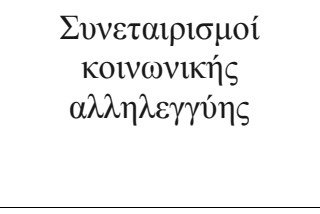 & 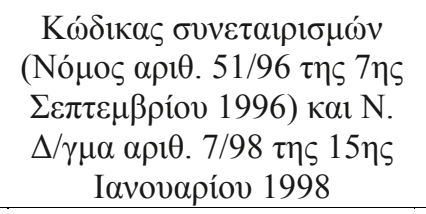 & 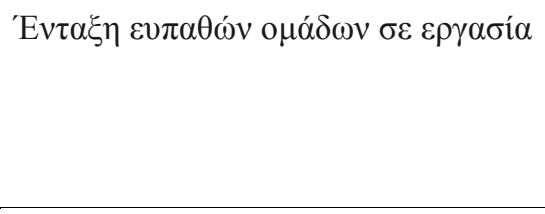 \\
\hline 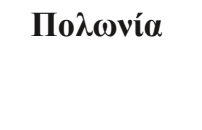 & 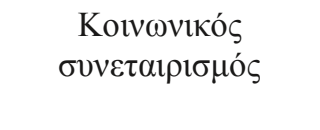 & 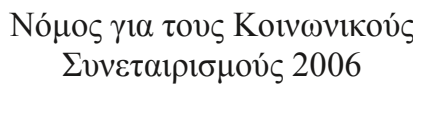 & 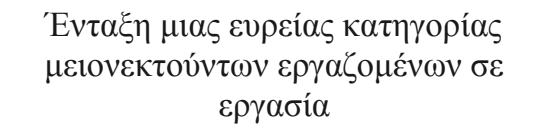 \\
\hline 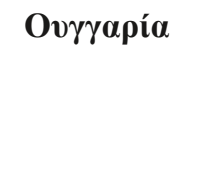 & 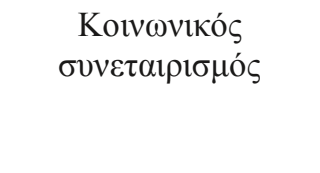 & Nópos 2006 . X & 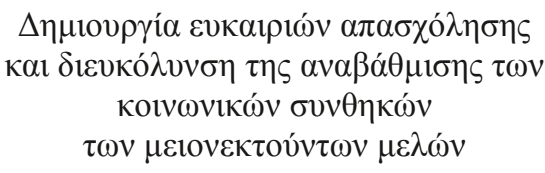 \\
\hline$E \lambda \lambda \alpha ́ \delta \alpha$ & 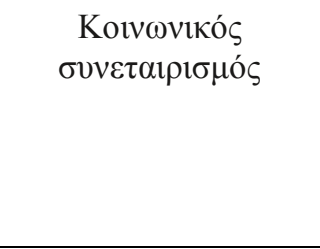 & 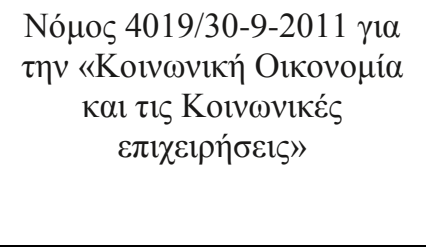 & 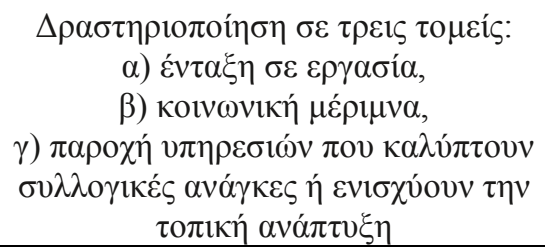 \\
\hline
\end{tabular}

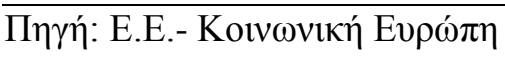

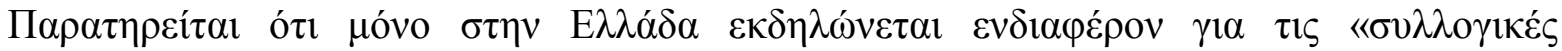

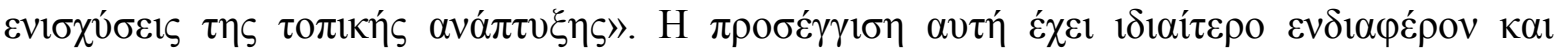

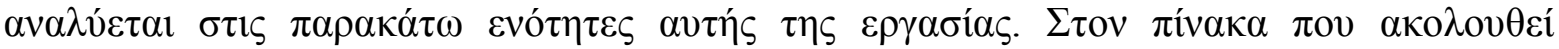

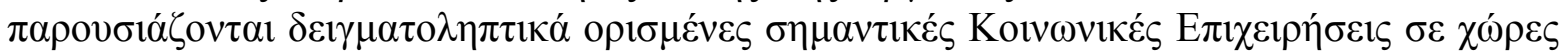
$\tau \eta \varsigma$ E.E.

Пívakas 8

\begin{tabular}{|c|c|}
\hline 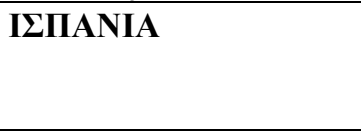 & 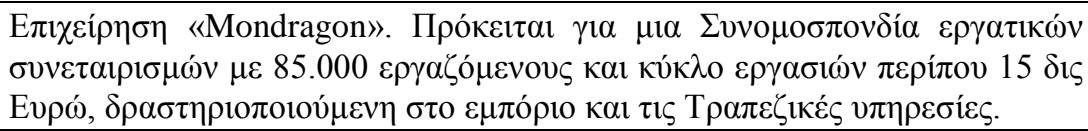 \\
\hline IP $\Lambda \mathbf{A N} \Delta \mathbf{I A}$ & 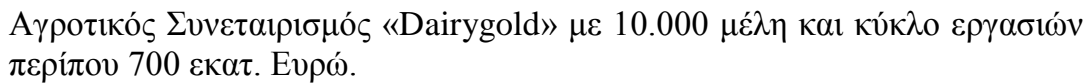 \\
\hline МЕГААН ВРЕTANIA & 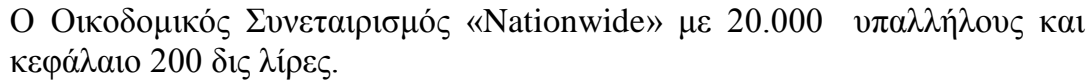 \\
\hline इОYНАIA & 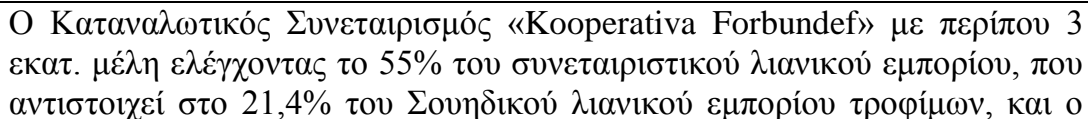 \\
\hline
\end{tabular}




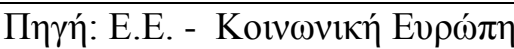

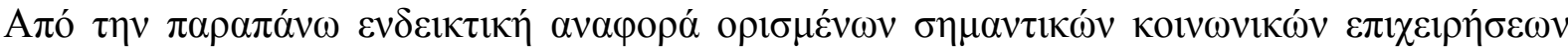

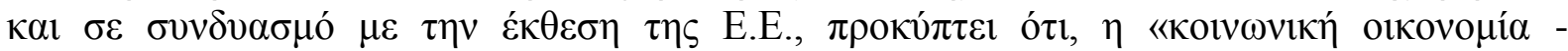

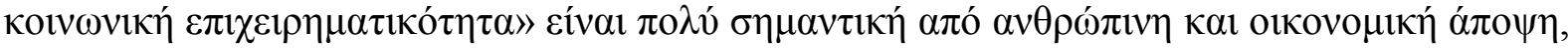

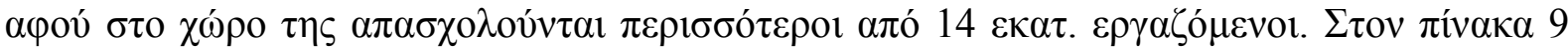

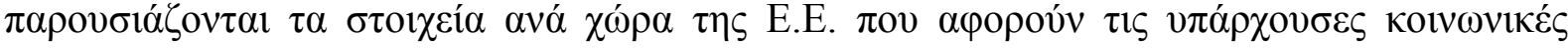

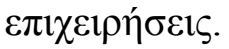

Пívaкas 9

\begin{tabular}{|c|c|c|c|c|}
\hline 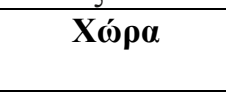 & 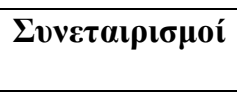 & 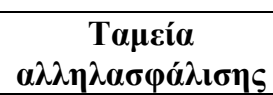 & 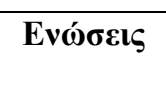 & 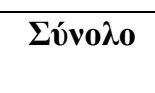 \\
\hline B'́́’ $\lambda \gamma 10$ & 17.047 & 12.864 & 249.700 & 279.611 \\
\hline$\Gamma \alpha \lambda \lambda i ́ \alpha$ & 439.720 & 110.100 & 1.435 .330 & 1.985 .150 \\
\hline I $\rho \lambda \alpha v \delta i ́ \alpha$ & 35.992 & 650 & 118.664 & 155.306 \\
\hline I $\tau \alpha \lambda i ́ \alpha$ & 837.024 & - & 499.389 & 1.336 .413 \\
\hline 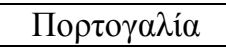 & 51.000 & - & 159.950 & 210.950 \\
\hline 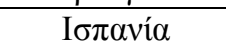 & 488.606 & 3.548 & 380.060 & 872.214 \\
\hline 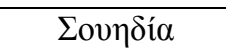 & 99.500 & 11.000 & 95.197 & 205.697 \\
\hline 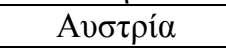 & 62.145 & 8.000 & 190.000 & 260.145 \\
\hline$\Delta \alpha v^{\prime} \alpha$ & 39.107 & 1.000 & 120.657 & 160.764 \\
\hline$\Phi_{1} \lambda \alpha v \delta i ́ \alpha$ & 95.000 & 5.405 & 74.992 & 175.397 \\
\hline 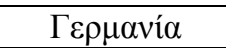 & 466.900 & 150.000 & 1.414 .937 & 2.031 .837 \\
\hline$E \lambda \lambda \alpha ́ \delta \alpha$ & 12.345 & 489 & 57.000 & 69.834 \\
\hline$\Lambda$ ov $\xi \varepsilon \mu \beta$ ои́рүо & 748 & - & 6.500 & 7.248 \\
\hline 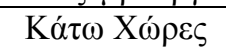 & 110.710 & - & 661.400 & 772.110 \\
\hline 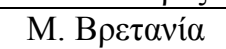 & 190.458 & 47.818 & 1.473 .000 & 1.711 .276 \\
\hline 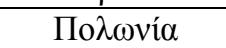 & 469.179 & - & 60.000 & 529.179 \\
\hline
\end{tabular}

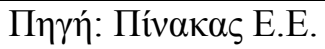

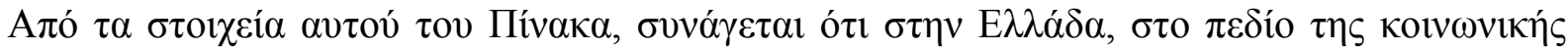

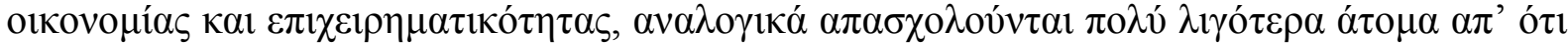

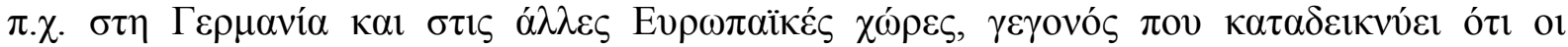

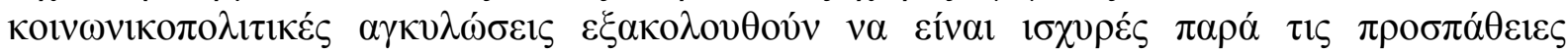

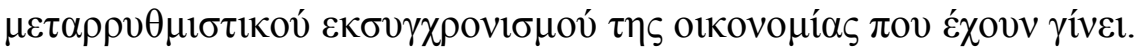

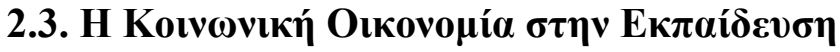

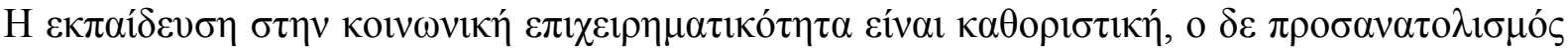

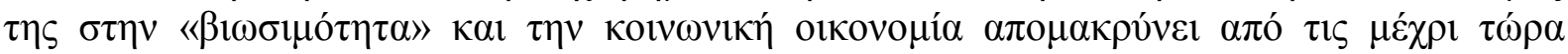

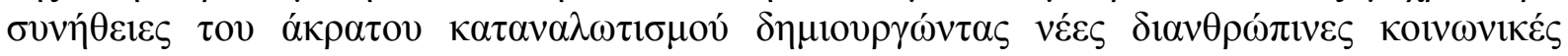

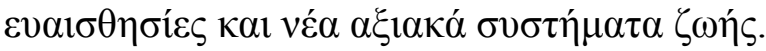

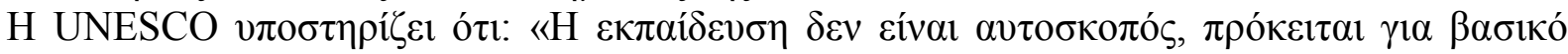

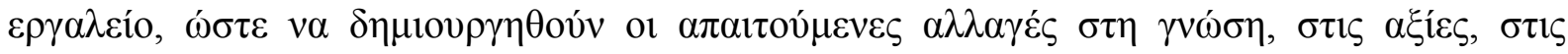

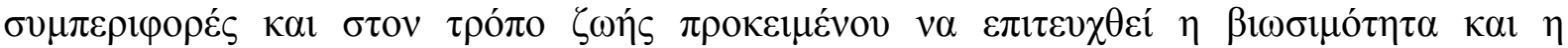
$\sigma \tau \alpha \theta \varepsilon \rho o ́ \tau \eta \tau \alpha »$.

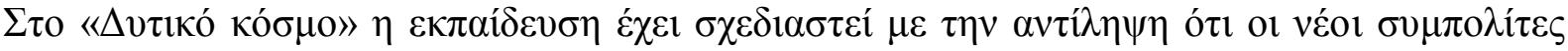

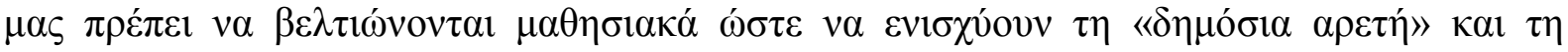

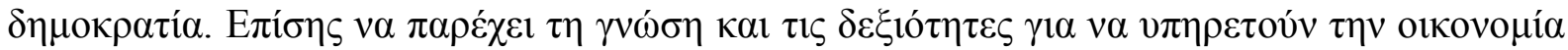

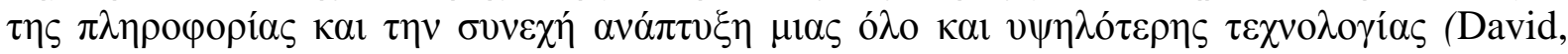
2006). 


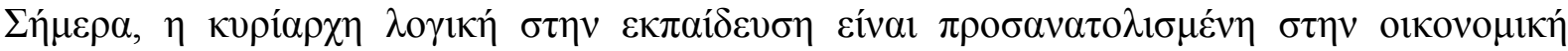

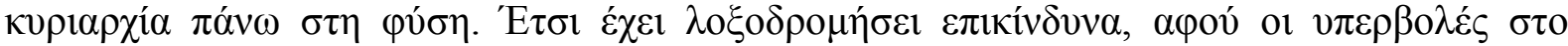

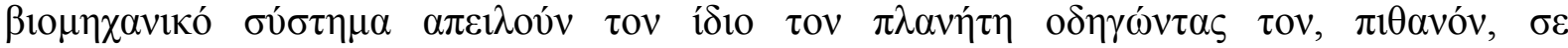

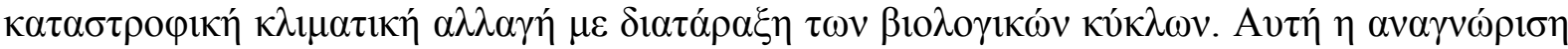

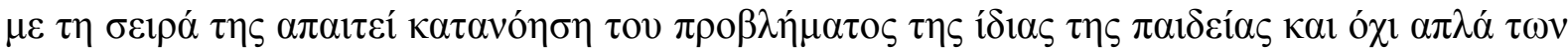

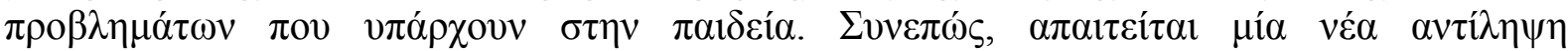

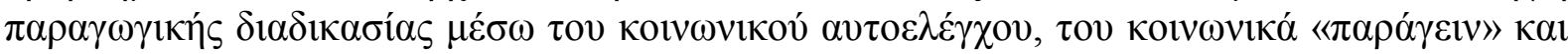

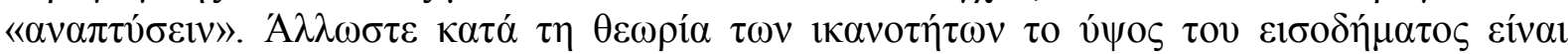

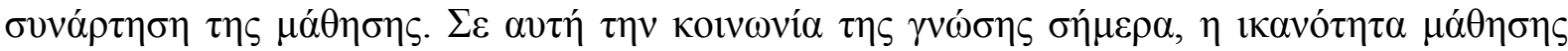

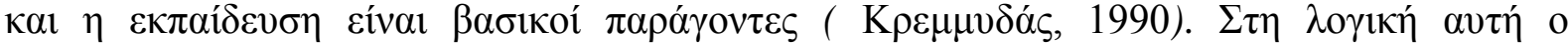

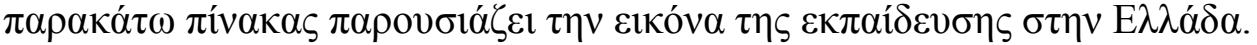

Пívaкas 10

\begin{tabular}{|c|c|c|c|c|c|c|c|c|c|c|}
\hline 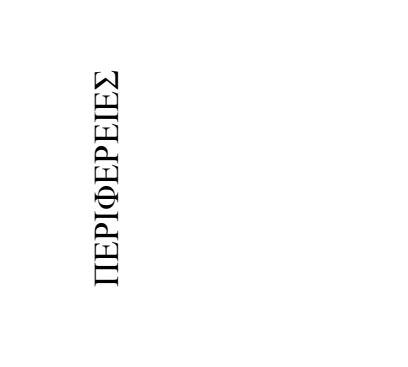 & 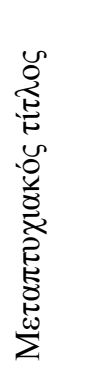 & 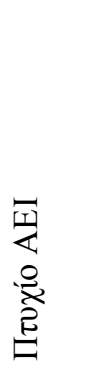 & 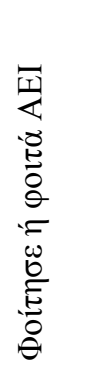 & 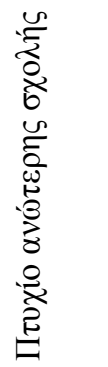 & 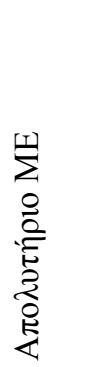 & 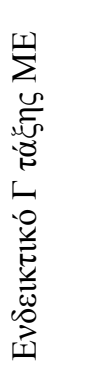 & 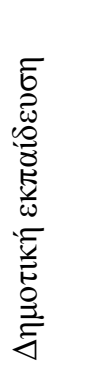 & 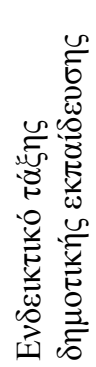 & 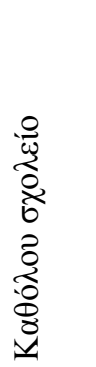 & 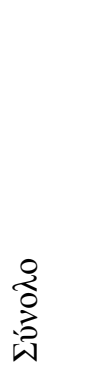 \\
\hline $\begin{array}{l}\text { AN. MAKE } \triangle \mathrm{ONIA} \mathrm{-} \\
\text { @PAKH }\end{array}$ & 0 & 3,43 & 3,67 & 3,27 & 3,86 & 4,58 & 8,37 & 11,7 & 22,77 & 6,45 \\
\hline KENTPIKH MAKE $\triangle$ ONIA & 10,77 & 15,75 & 12,65 & 14,08 & 15,59 & 18,74 & 18,44 & 18,32 & 10,64 & 17,03 \\
\hline$\triangle \mathrm{YTIKH}$ MAKE $\triangle \mathrm{ONIA}$ & 0,62 & 1,81 & 0 & 2,08 & 3,07 & 2,48 & 3,34 & 3,53 & 2,1 & 2,67 \\
\hline$\Theta \mathrm{E} \Sigma \Sigma \mathrm{A} \Lambda \mathrm{IA}$ & 1,23 & 5,23 & 1,63 & 4,74 & 5,01 & 6,78 & 8,48 & 9,26 & 10,4 & 6,92 \\
\hline HПЕIPО $\Sigma$ & 0,62 & 2,34 & 2,45 & 1,54 & 1,79 & 1,77 & 3,31 & 4,52 & 5,7 & 2,67 \\
\hline 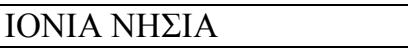 & 0 & 1,15 & 0,82 & 1,72 & 1,34 & 1,86 & 2,53 & 2,5 & 3,22 & 1,97 \\
\hline$\Delta \mathrm{YTIKH} \mathrm{E} \Lambda \Lambda \mathrm{A} \Delta \mathrm{A}$ & 5,23 & 5,18 & 2,45 & 5,21 & 5,39 & 6,27 & 7,63 & 6,41 & 6,93 & 6,44 \\
\hline$\Sigma$ TEPEA E $\Lambda \Lambda \mathrm{A} \Delta \mathrm{A}$ & 0 & 2,87 & 1,63 & 1,9 & 3,52 & 3,99 & 6,79 & 7,38 & 5,07 & 4,98 \\
\hline ПЕ $\Lambda$ OПONNH $\Sigma \mathrm{O} \Sigma$ & 0,92 & 3,07 & 0,41 & 2,19 & 4,77 & 7,4 & 7,19 & 7,65 & 4,95 & 5,8 \\
\hline ATTIKH & 75,38 & 53,37 & 70,61 & 55,37 & 49,36 & 37,36 & 22,65 & 15,75 & 17,94 & 35,75 \\
\hline ВОРЕIO АГАIО & 1,54 & 1,63 & 0,82 & 2,12 & 1,49 & 1,84 & 1,57 & 2,88 & 1,73 & 1,69 \\
\hline NOTIO АIГAIO & 0,92 & 1,37 & 2,04 & 1,69 & 2,29 & 2,41 & 2,6 & 2,85 & 0,99 & 2,29 \\
\hline KPHTH & 2,77 & 2,79 & 0,82 & 4,09 & 3,54 & 4,52 & 7,1 & 7,51 & 8,17 & 5,34 \\
\hline$\Sigma \mathrm{YNO} \Lambda \mathrm{O}$ & 100 & 100 & 100 & 100 & 100 & 100 & 100 & 100 & 100 & 100 \\
\hline
\end{tabular}

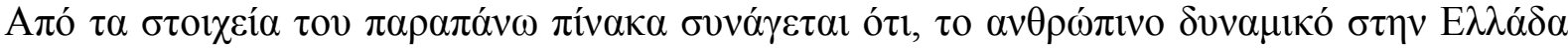

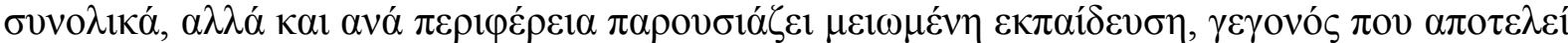

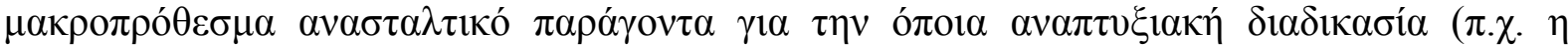

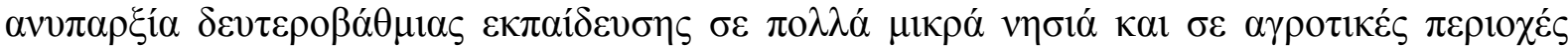

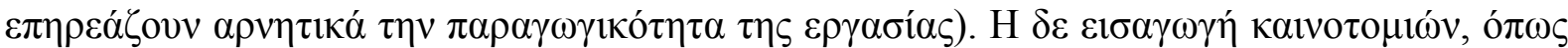

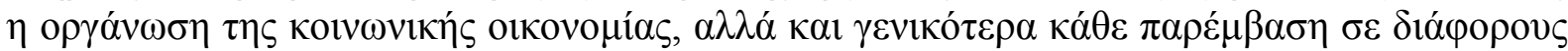

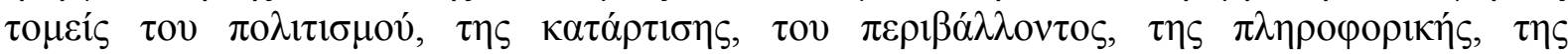

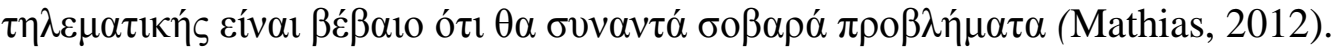




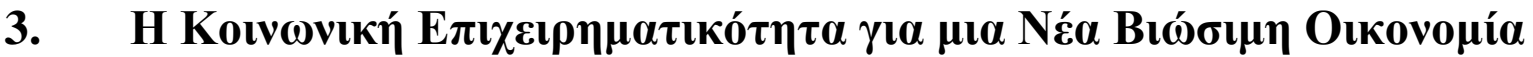

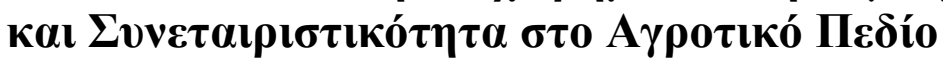

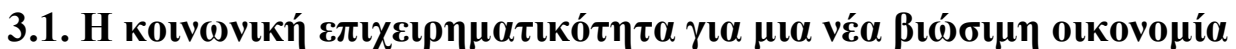

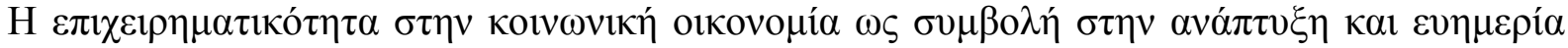

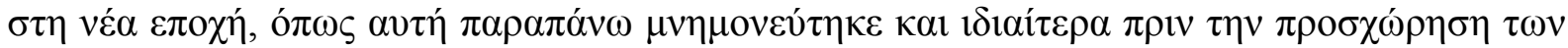

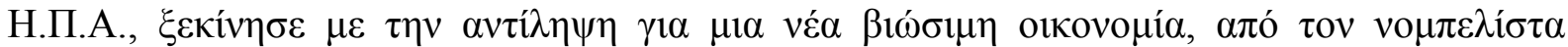

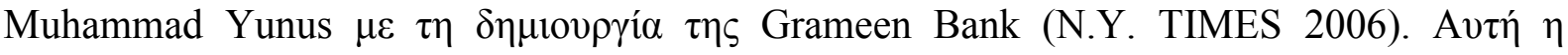

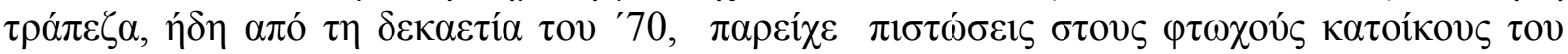

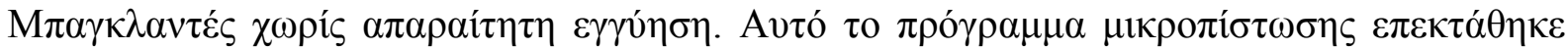

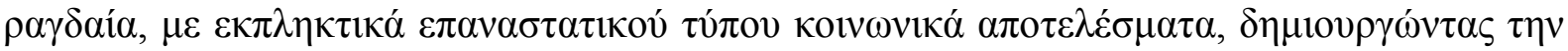

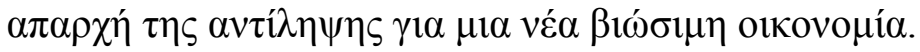

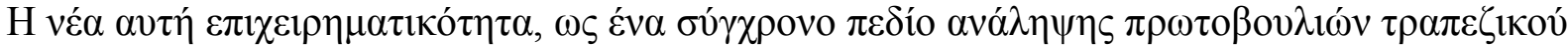

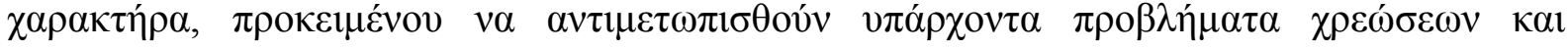

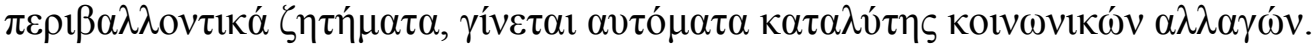

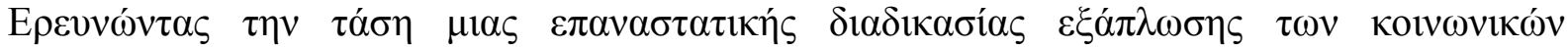

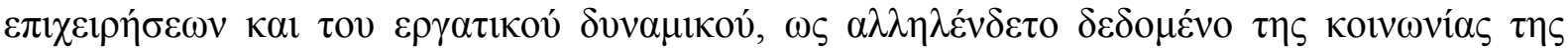

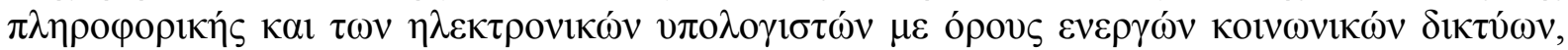

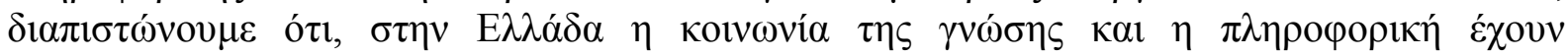

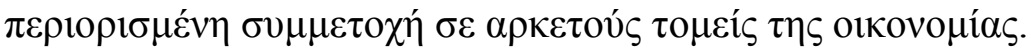

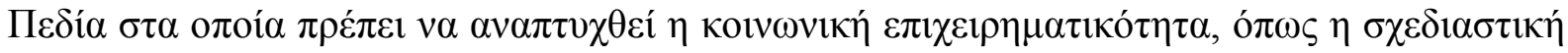

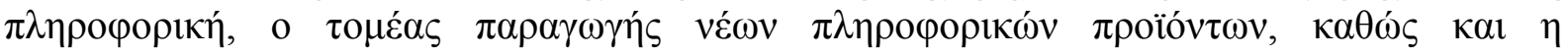

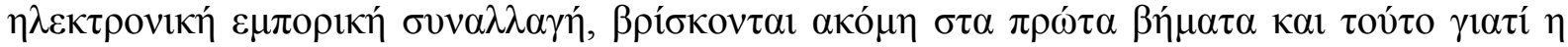

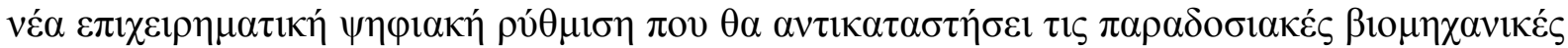

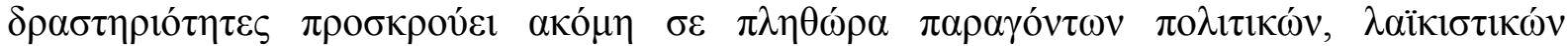

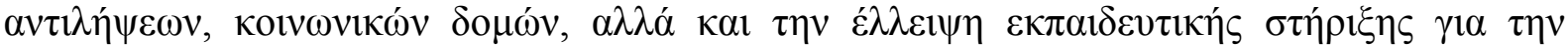

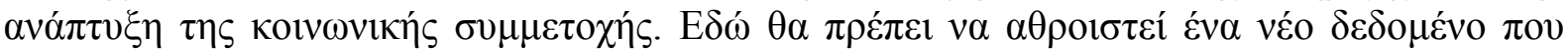

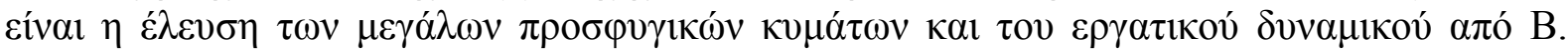

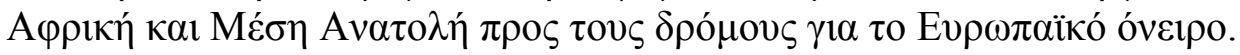

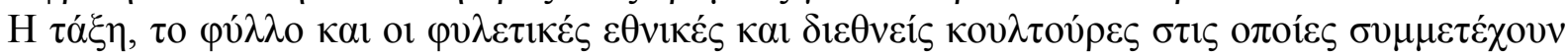

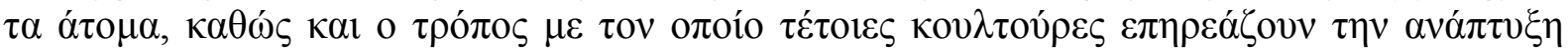

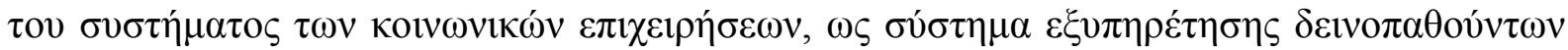

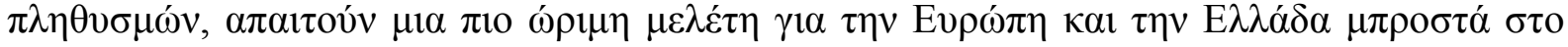

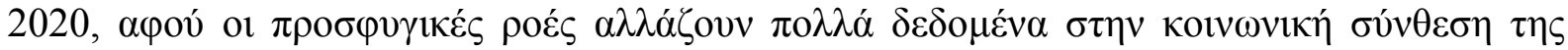

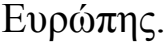

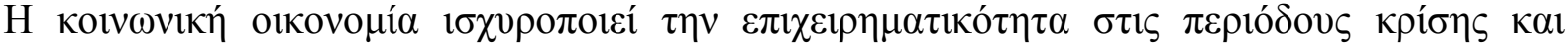

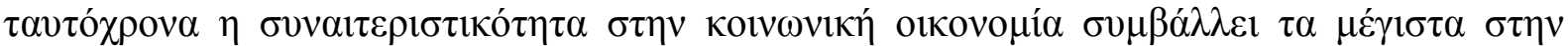

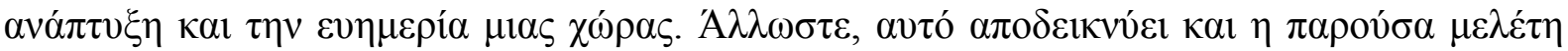

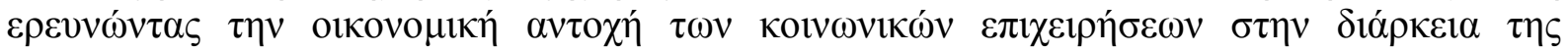

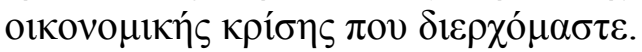

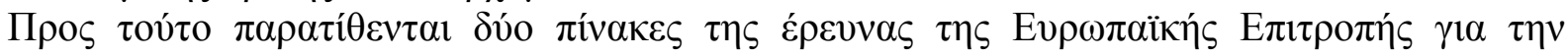

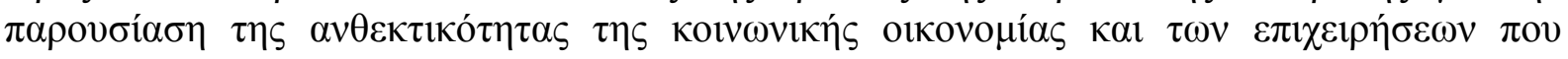

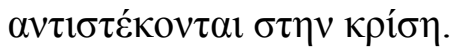




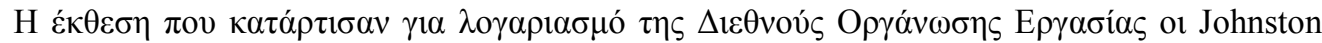

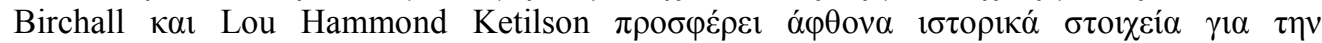

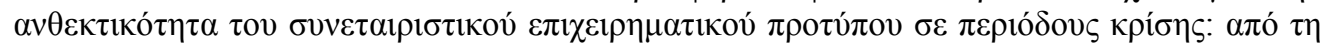

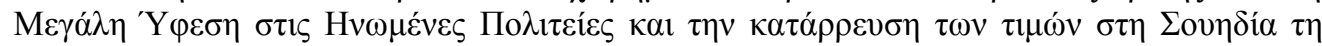

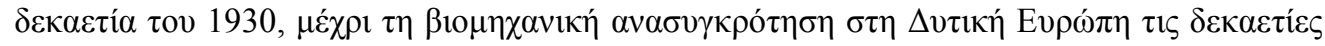

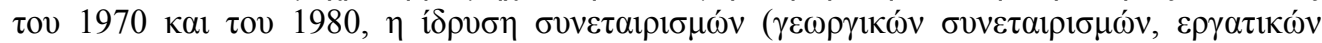

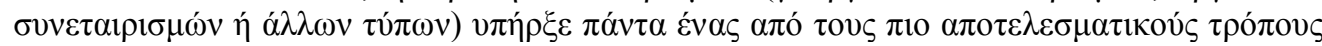

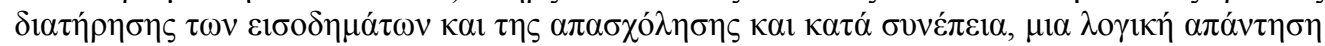

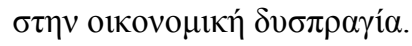

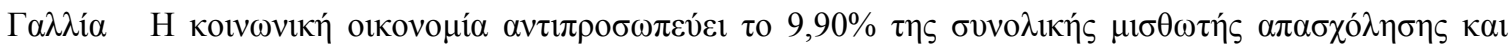

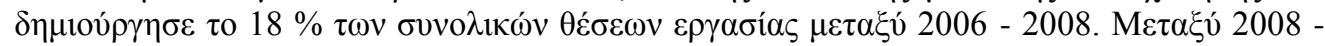

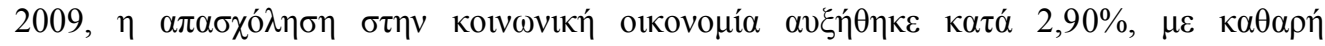

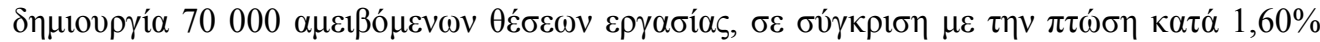

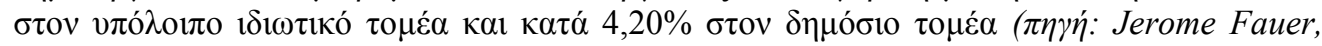
The social economy: Preparing the ground for innovative responses to current challenges, $\sigma \chi \dot{\varepsilon} \delta \imath o \dot{\varepsilon} \kappa \theta \varepsilon \sigma \eta \varsigma)$.

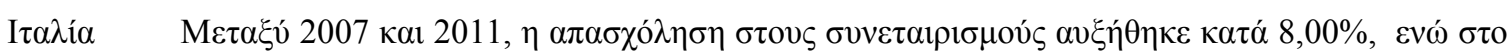
бúvo

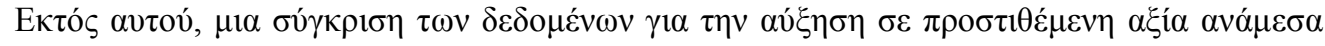

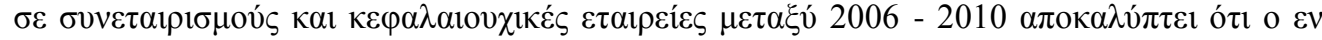

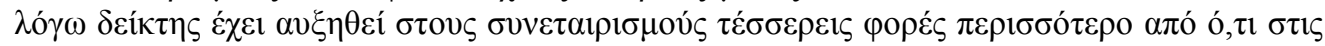

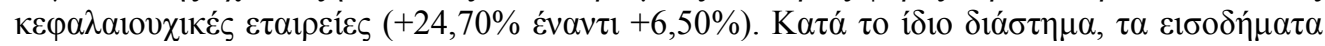

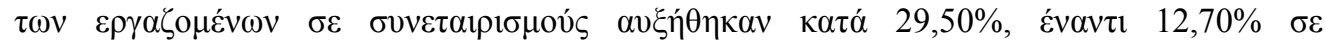

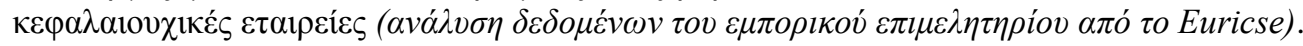

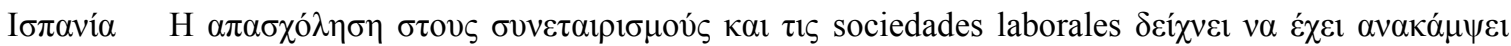

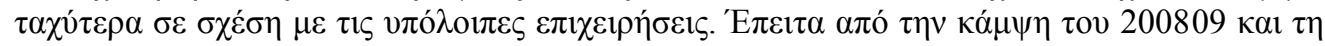

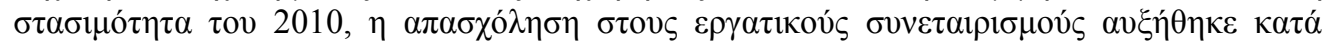

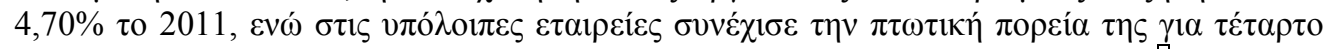

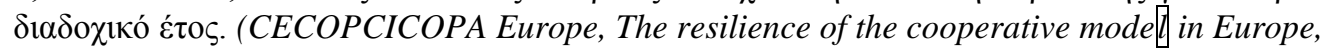
Iov́vios 2012).

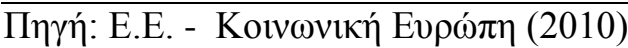

\section{ПívaKas 12}

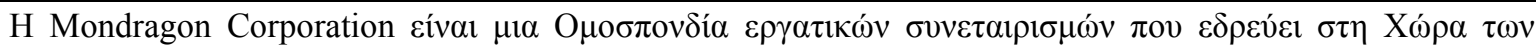

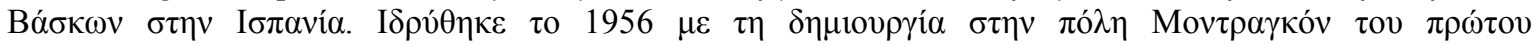

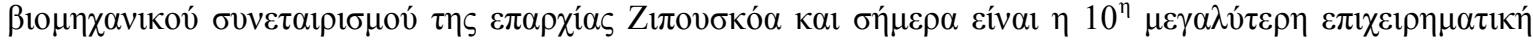

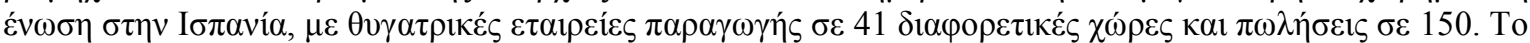

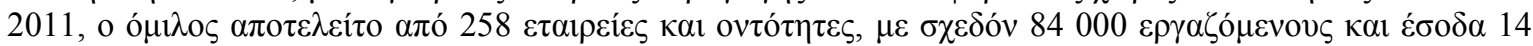

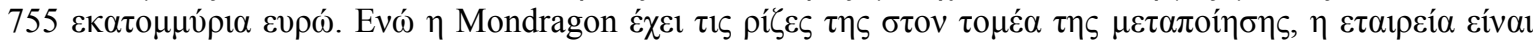

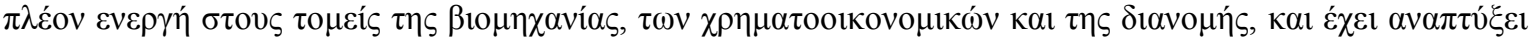

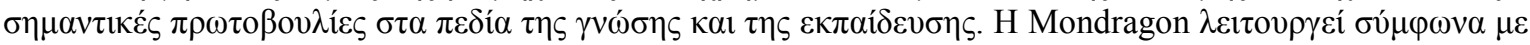

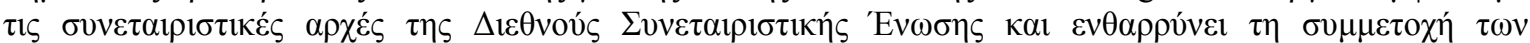

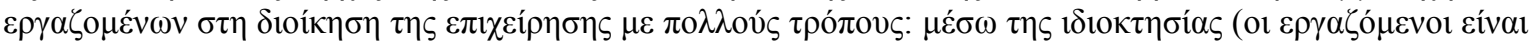

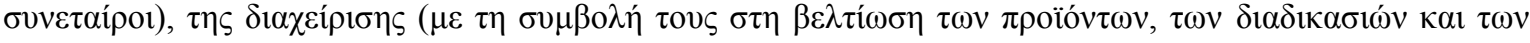

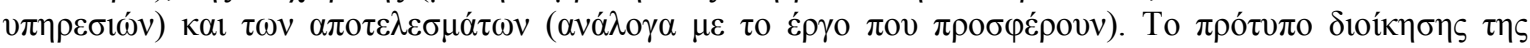

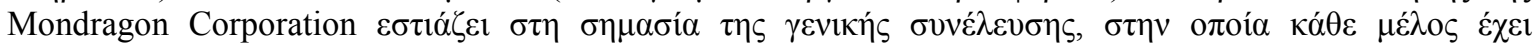

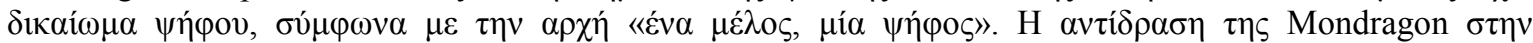

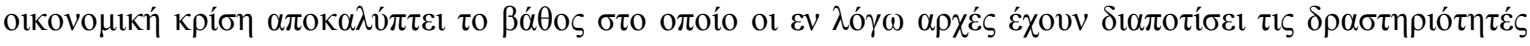
$\tau \eta \varsigma$. 


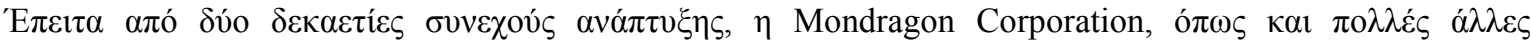

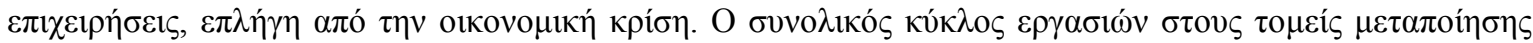

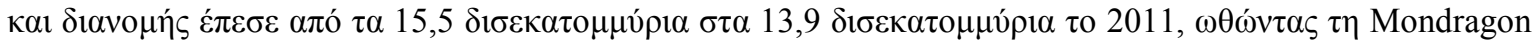

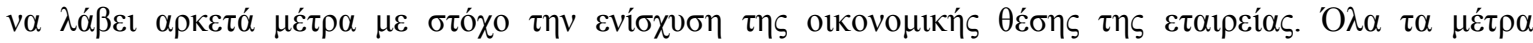

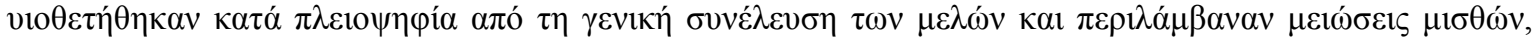

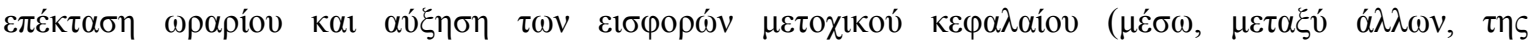

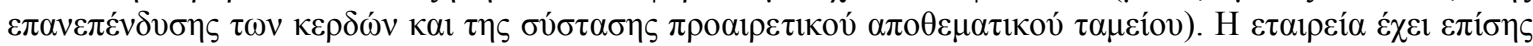

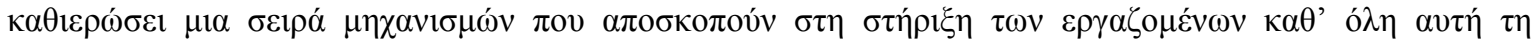

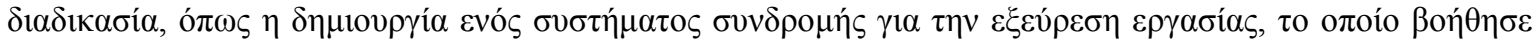

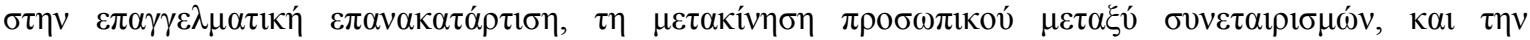

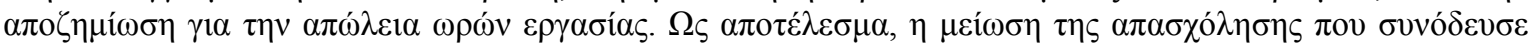

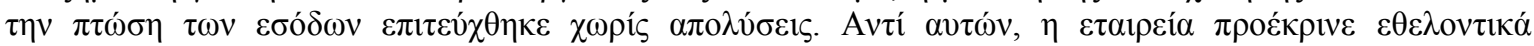

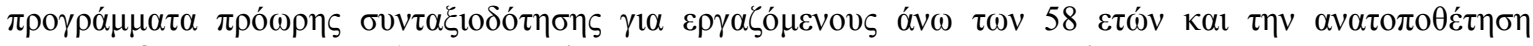

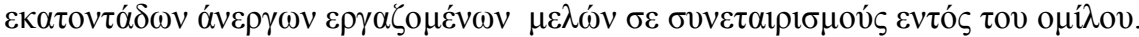

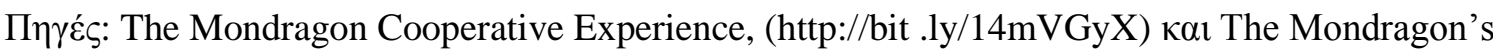
Corporate Management Model (http://bit .ly/ZYV1Oq)

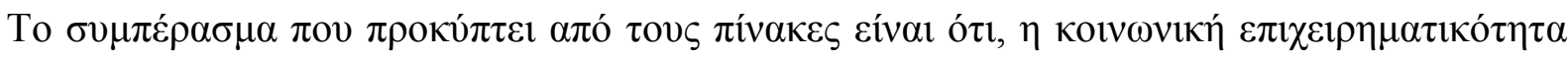

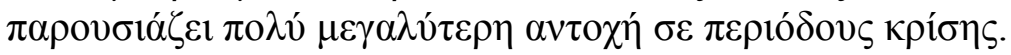

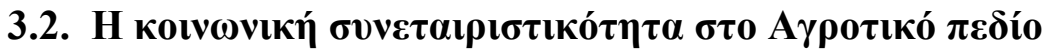

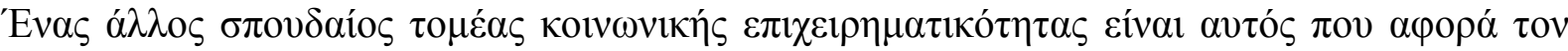

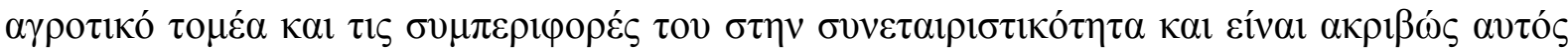

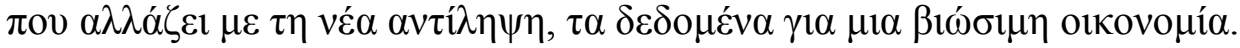

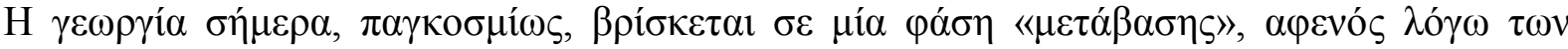

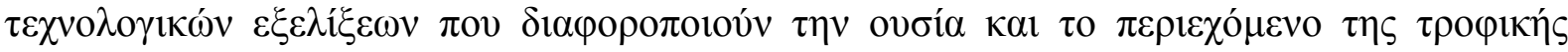

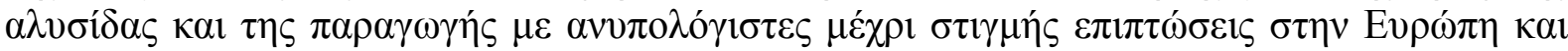

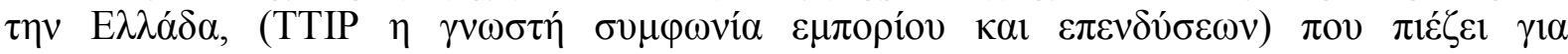

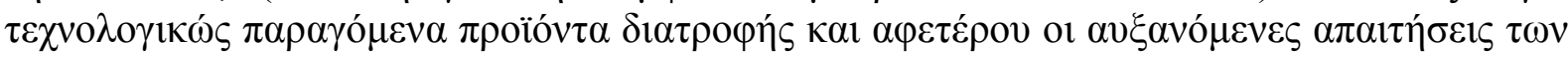

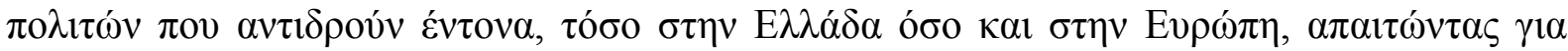

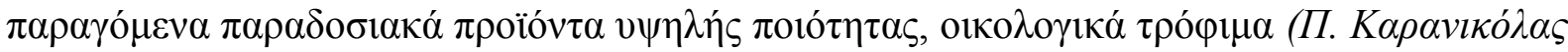

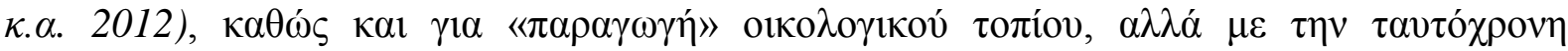

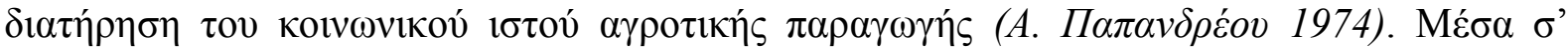

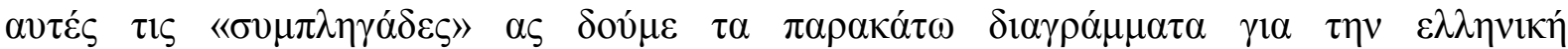
$\pi \rho \alpha \gamma \mu \alpha \tau 1 \kappa o ́ \tau \eta \tau \alpha$.

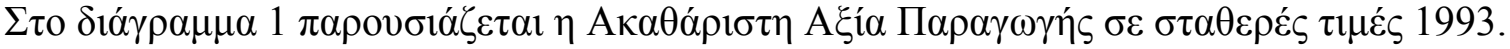

$\Delta \mathbf{l}^{\prime} \gamma \rho \alpha \mu \mu \alpha 1$

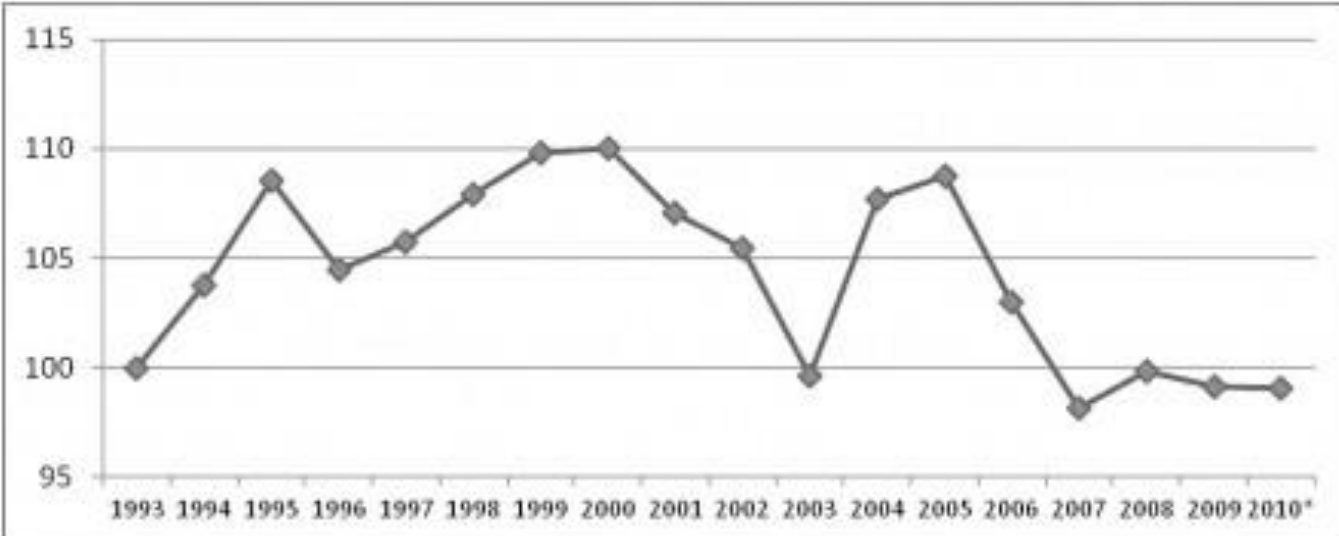

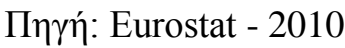




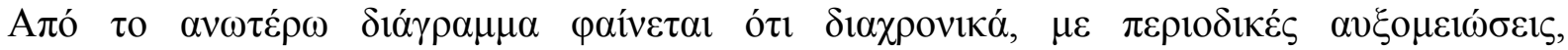

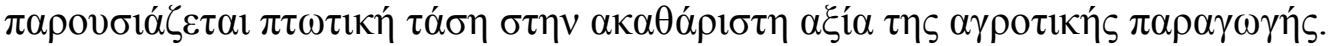

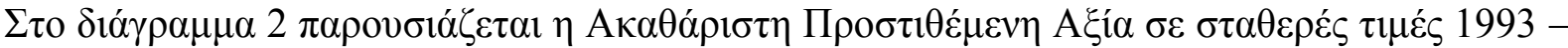
2010 .

$\Delta{ }^{\prime \alpha} \gamma \rho \alpha \mu \mu \alpha 2$

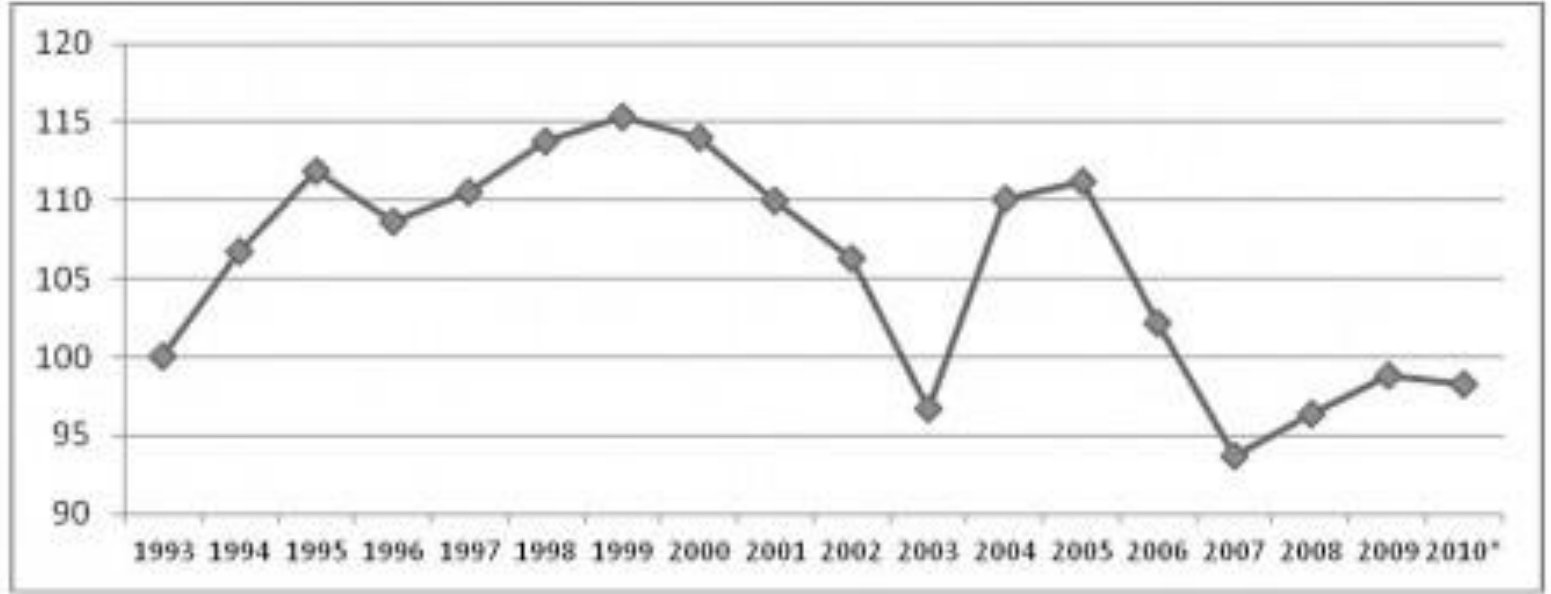

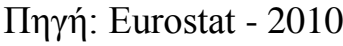

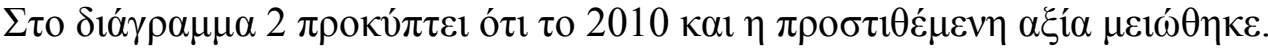

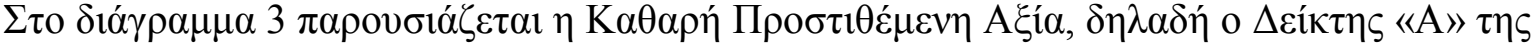
Eurostat

$\Delta \mathbf{l}^{\prime} \gamma \rho \alpha \mu \mu \alpha 3$

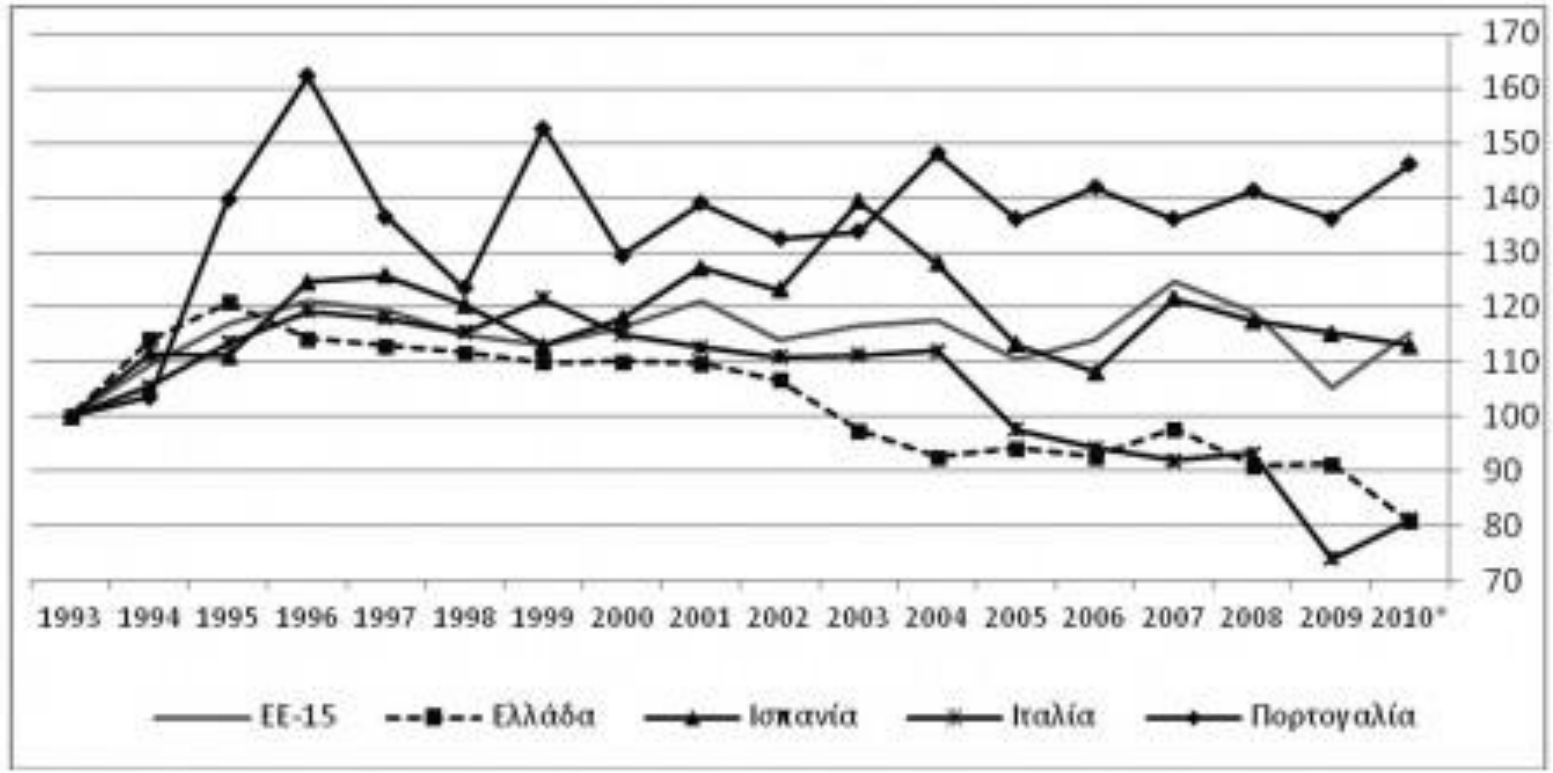

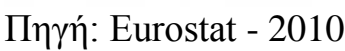

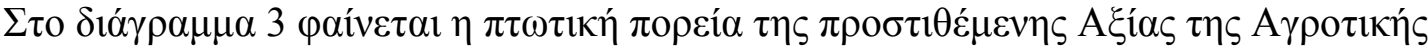

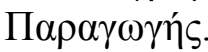




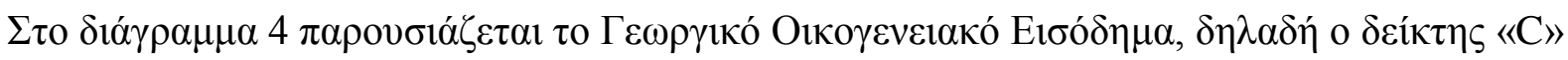
$\tau \eta \varsigma$ Eurostat.

\section{$\Delta{ }^{\prime} \alpha ́ \gamma \rho \alpha \mu \mu \alpha 4$}

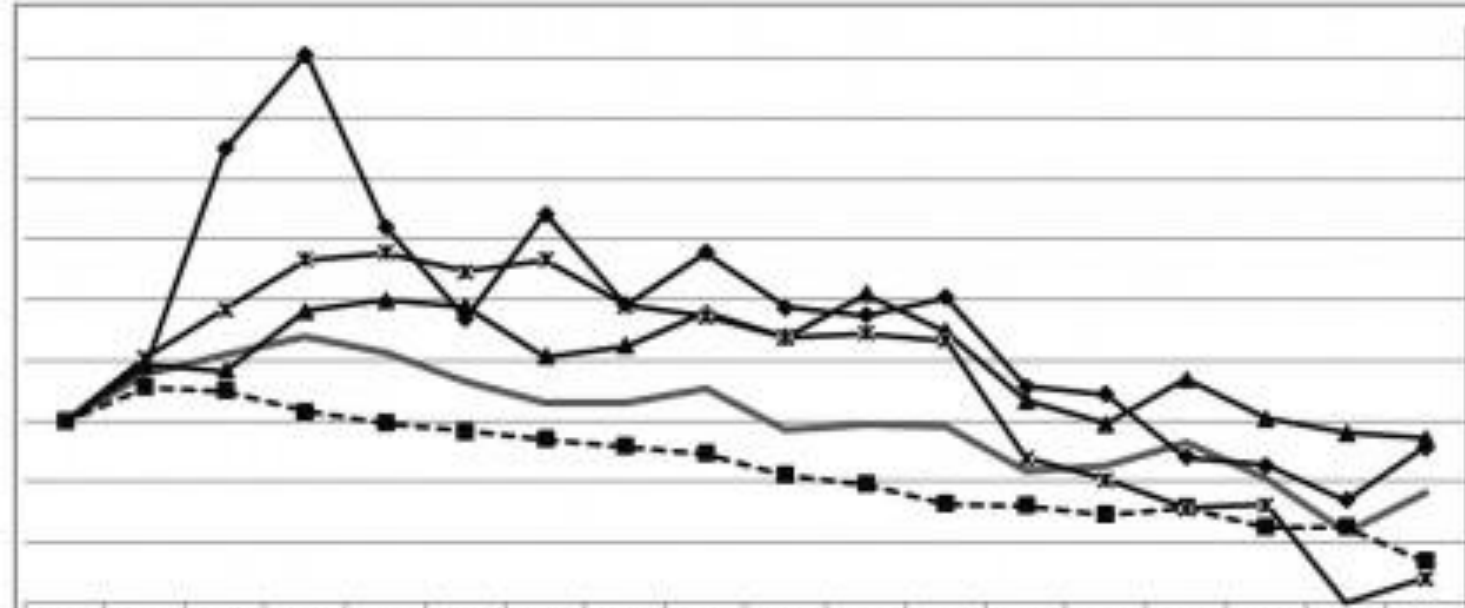

\section{0

$1993199419951996199719981999200020012002.2003200420052006 \quad 2007200820092010^{*}$

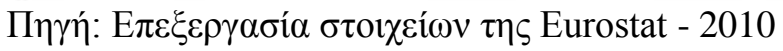

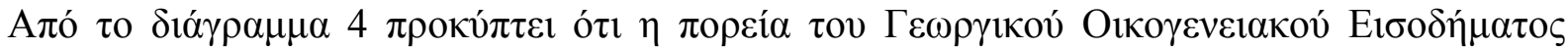

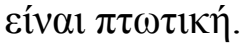

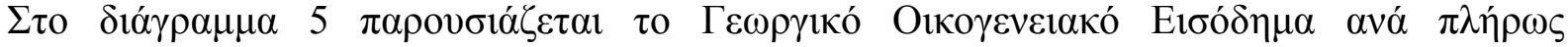

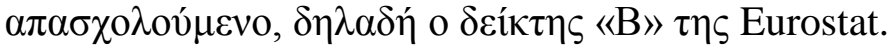

\section{$\Delta \mathrm{ló} \gamma \rho \alpha \mu \mu \alpha 5$}

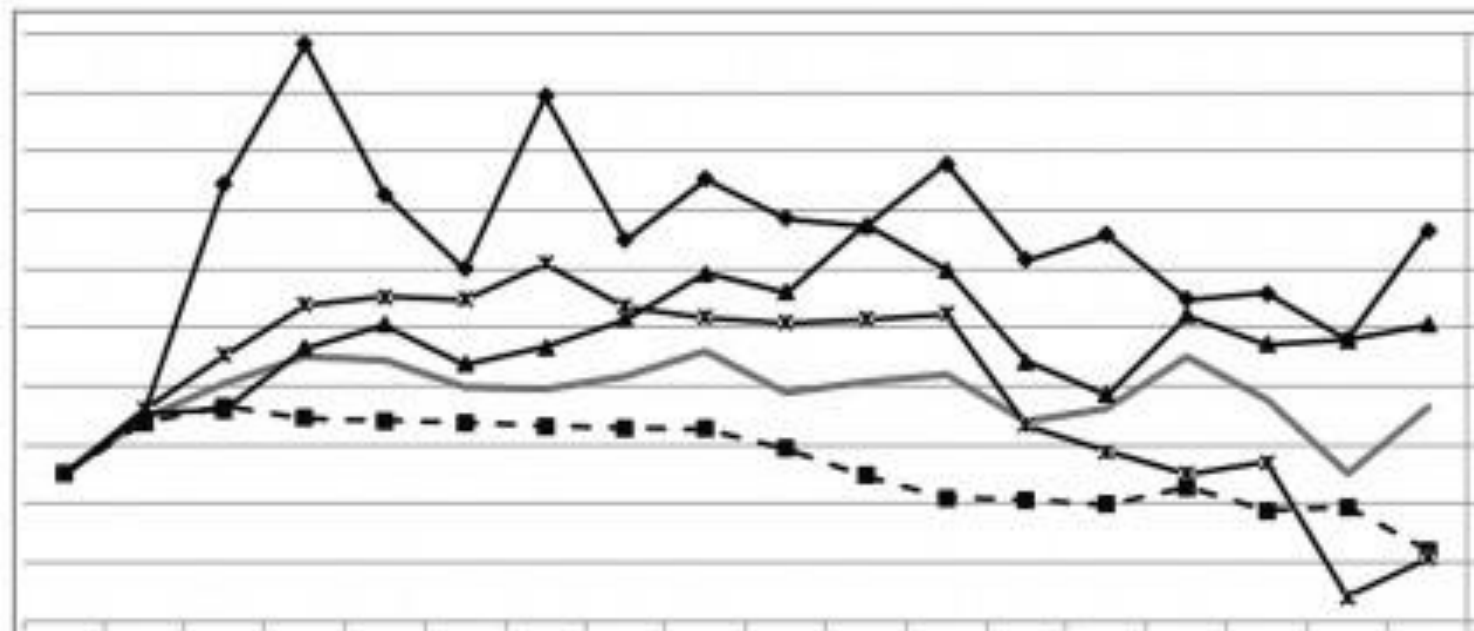

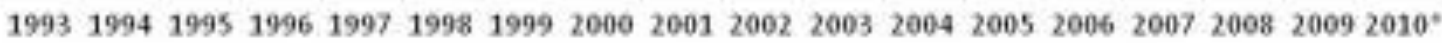

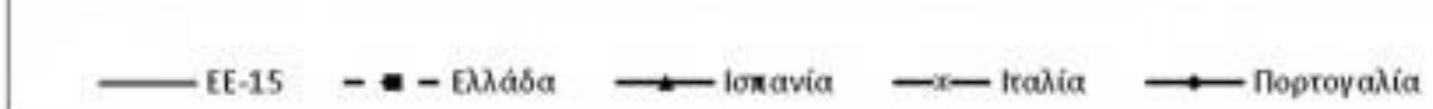

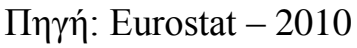




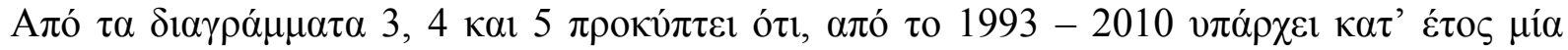

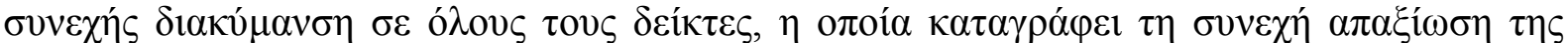

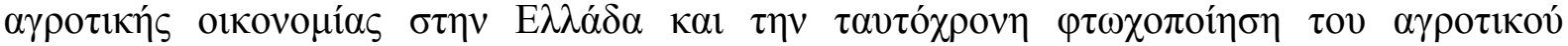

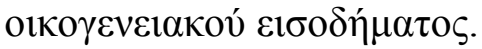

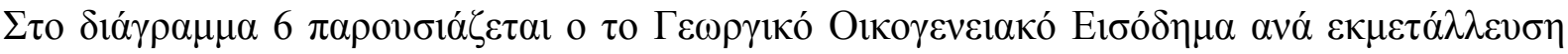

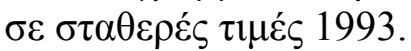

$\Delta{ }^{\prime} \alpha \gamma_{\rho} \alpha \mu \mu \alpha 6$

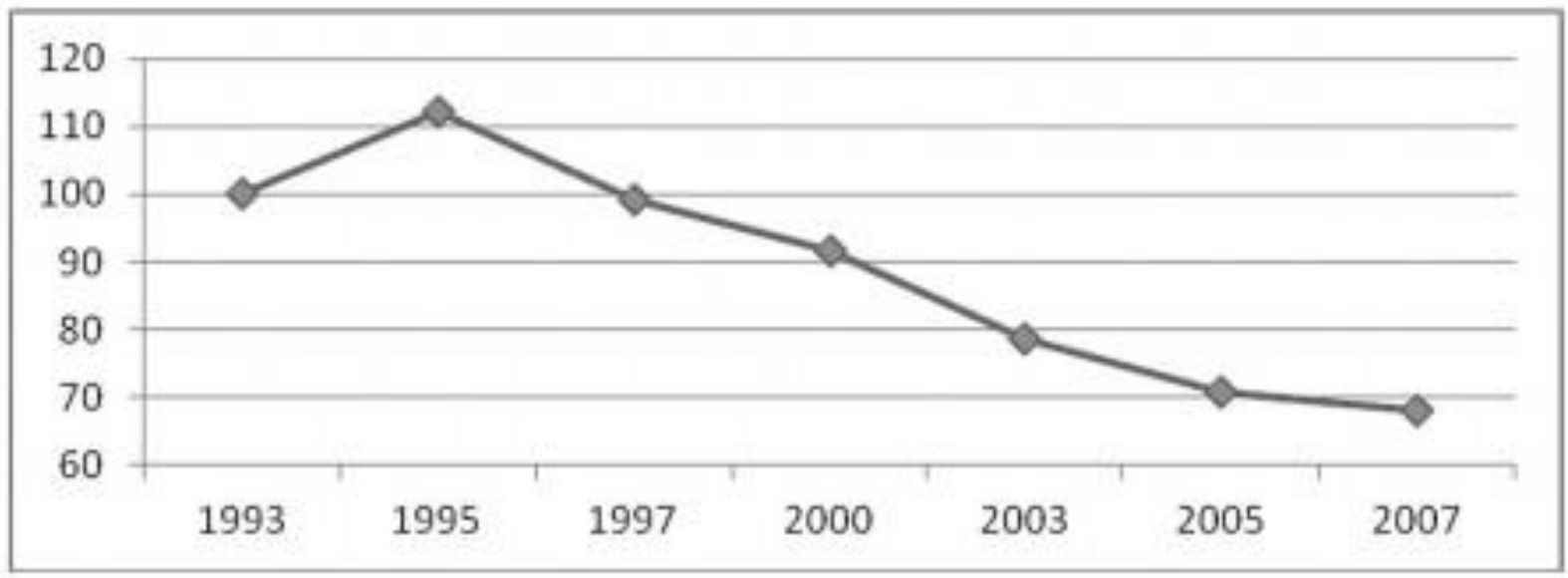

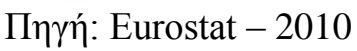

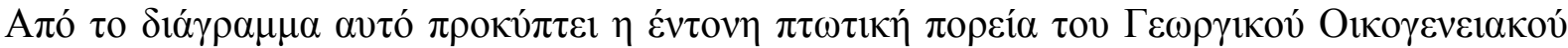

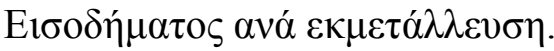

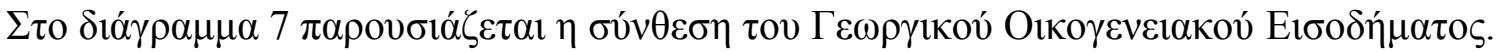

$\Delta \mathbf{l o ́}_{\gamma} \rho \alpha \mu \alpha \alpha$

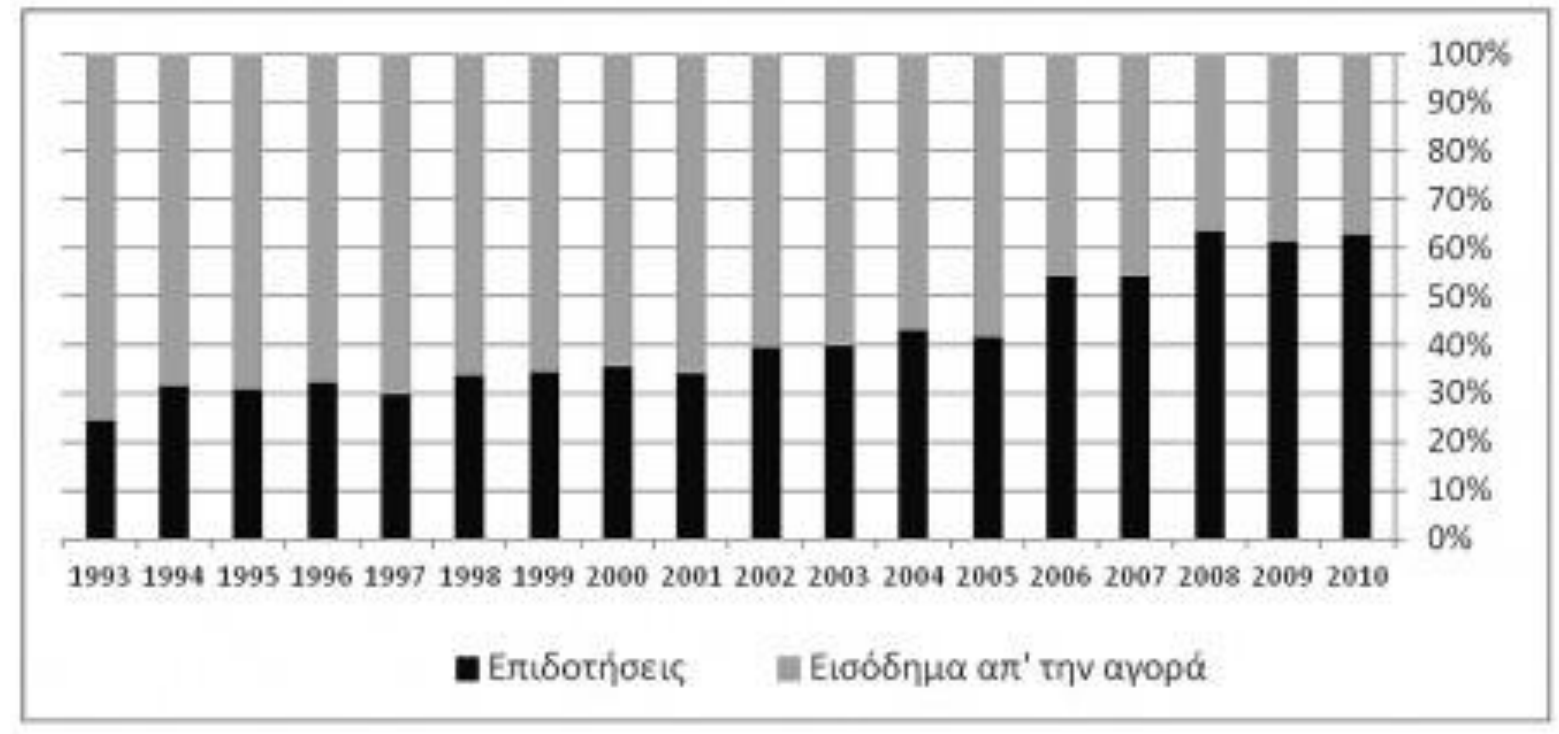

Пүүฑ์: Eurostat - 2010

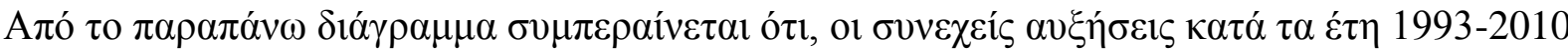

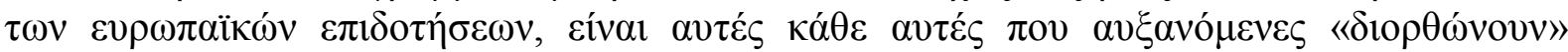

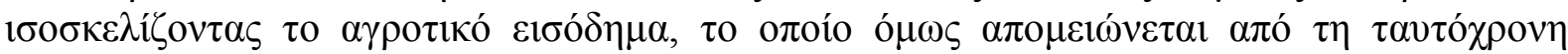

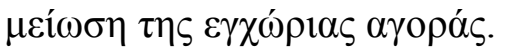




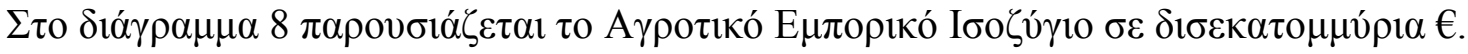

$\Delta \mathbf{l}^{\prime} \gamma \rho \alpha \mu \mu \alpha 8$

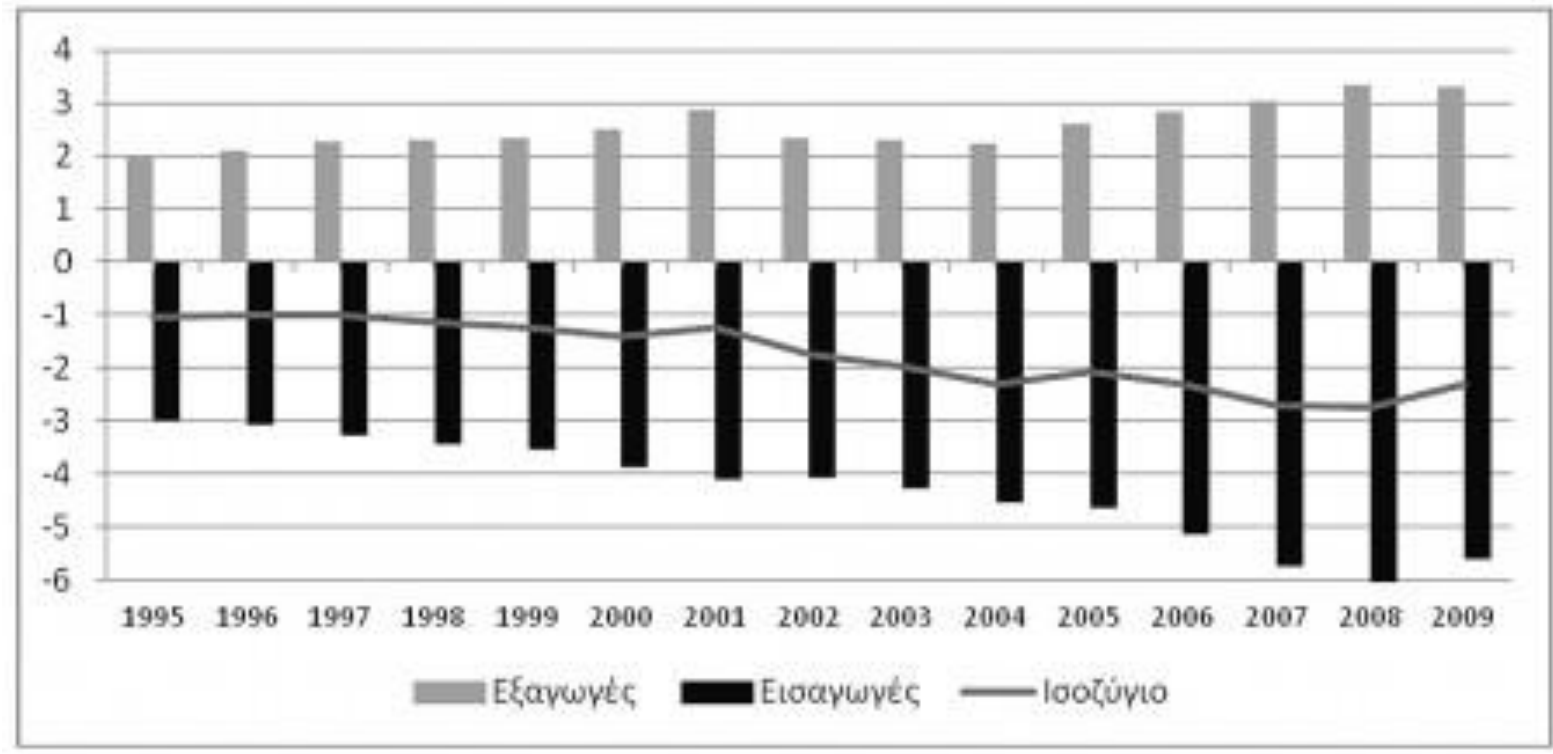

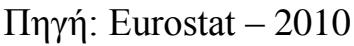

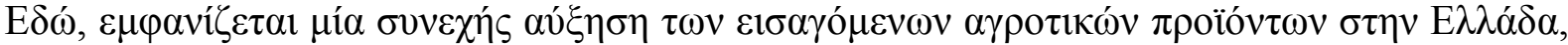

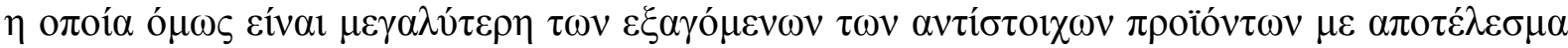

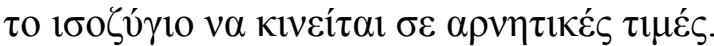

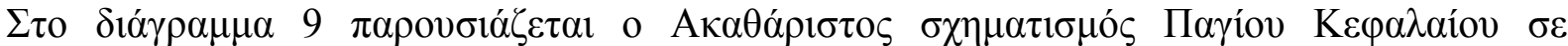

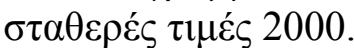

$\Delta \mathrm{l} \alpha \dot{\gamma} \rho \mu \mu \alpha 9$

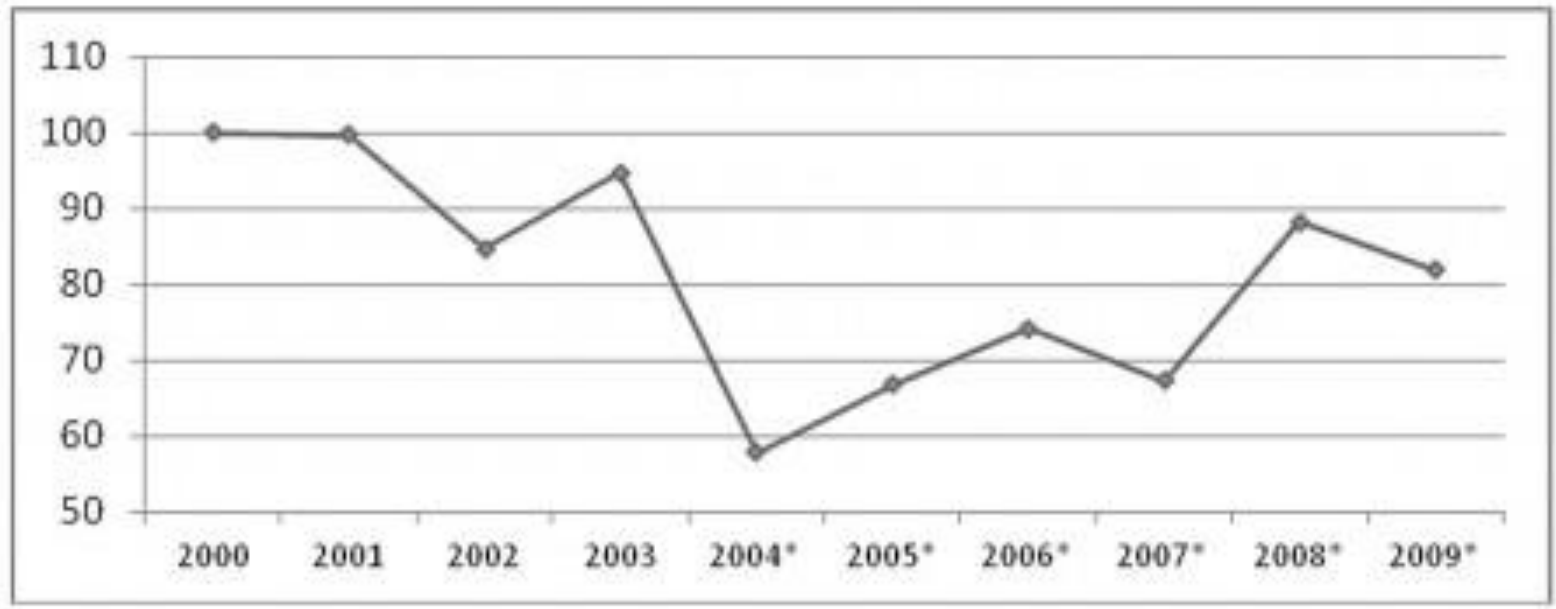

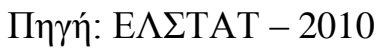

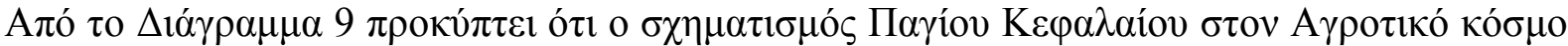

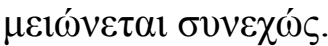

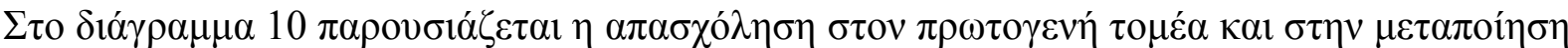

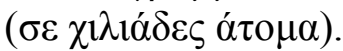




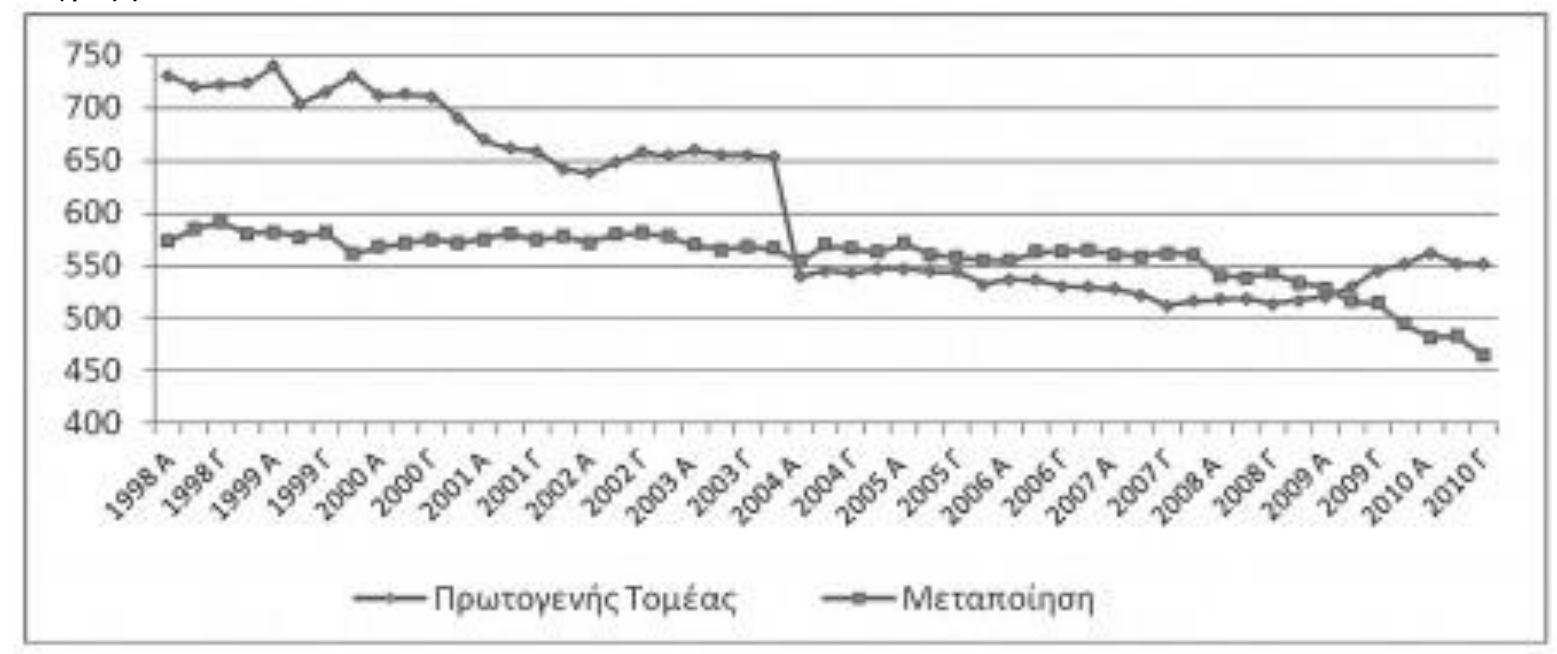

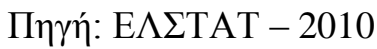

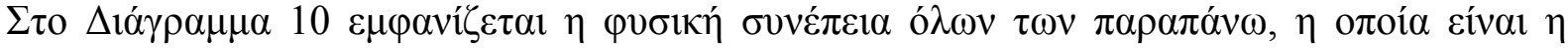

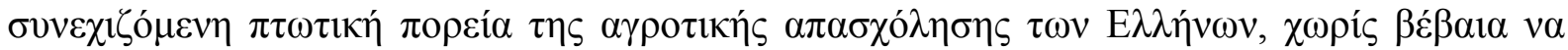

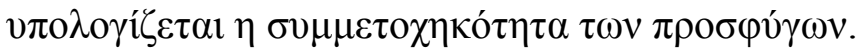

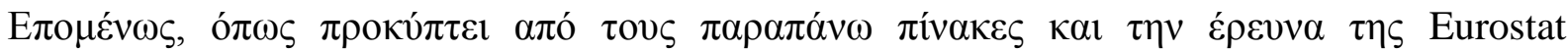

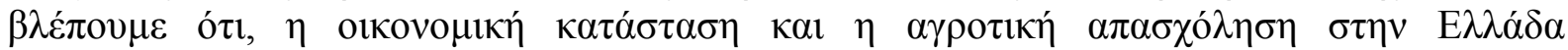

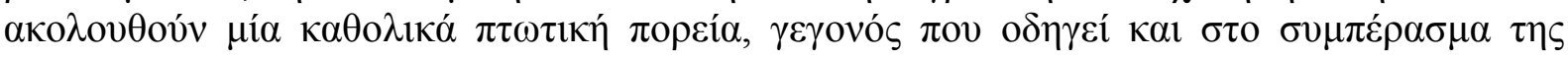

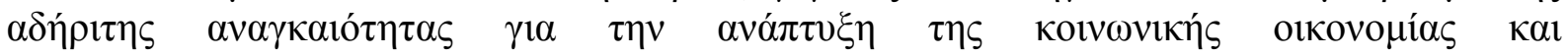

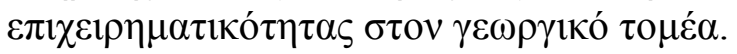

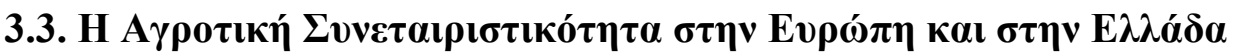

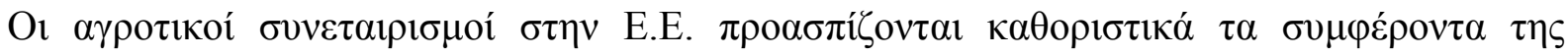

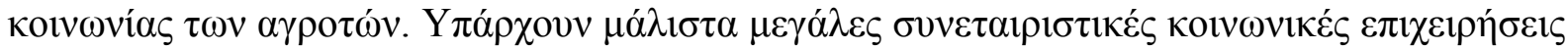

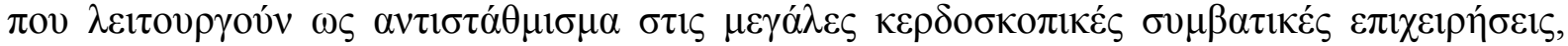

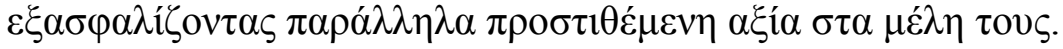

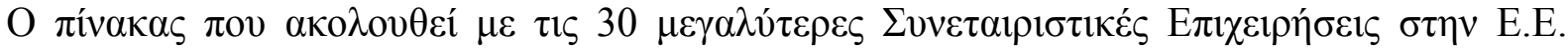

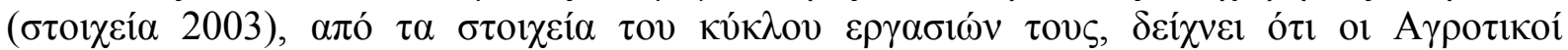

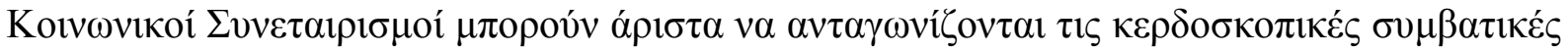

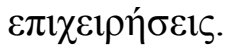

Пívakas 13

\begin{tabular}{|c|c|c|c|c|}
\hline & Е $\pi \omega v v \mu i^{\alpha} \alpha$ & 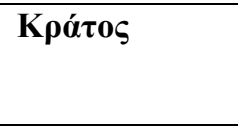 & 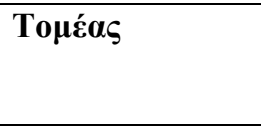 & 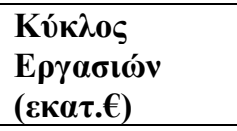 \\
\hline 1. & Metsaliitto & $\Phi ı v \lambda \alpha v \delta i ́ \alpha$ & 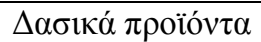 & 8.300 \\
\hline 2. & Bay Wa & $\Gamma \varepsilon \rho \mu \alpha v i ́ \alpha$ & $\mathrm{E} \varphi \delta^{\delta} 1 \alpha$ & 5.891 \\
\hline 3. & Arla Foods & 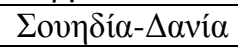 & 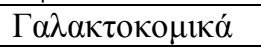 & 5.460 \\
\hline 4. & Danish Crown & $\Delta \alpha v i ́ \alpha$ & $\mathrm{K} \rho \varepsilon \dot{\alpha} \alpha \tau$ & 5.420 \\
\hline 5. & Friesland Coberco Dairy Foods & 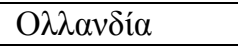 & 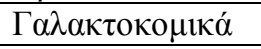 & 4.575 \\
\hline 6. & Kerry & I $\rho \lambda \alpha v \delta i ́ \alpha$ & 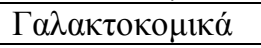 & 3.693 \\
\hline 7. & Campina & O $\lambda \lambda \alpha v \delta i ́ \alpha$ & 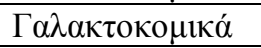 & 3.655 \\
\hline 8. & Agravis & $\Gamma \varepsilon \rho \mu \alpha v i ́ \alpha$ & E $\varphi$ ó $\delta 1 \alpha$ & 3.380 \\
\hline 9. & Svenska Lantmanen & $\Sigma o u \eta \delta i ́ \alpha$ & E $\varphi$ ó $\delta 1 \alpha$ & 3.100 \\
\hline 10. & Terrena & $\Gamma \alpha \lambda \lambda i ́ \alpha$ & E $\varphi \delta_{\delta 1 \alpha}$ & 2.973 \\
\hline 11. & Union IN VIVO & $\Gamma \alpha \lambda \lambda i ́ \alpha$ & $\Sigma ı \tau \eta \rho \alpha ́$, Е $\varphi o ́ \delta ı \alpha$ & 2.727 \\
\hline 12. & Humana Milchunion & $\Gamma \varepsilon \rho \mu \alpha v^{\prime} \alpha$ & $\Gamma \alpha \lambda \alpha \kappa \tau о \kappa о \mu 1 \kappa \alpha ́$ & 2.444 \\
\hline
\end{tabular}




\begin{tabular}{|c|c|c|c|c|}
\hline 13. & Nordmilch & 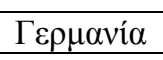 & 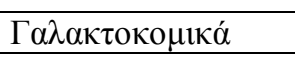 & 2.226 \\
\hline 14. & Glanbia & I $\rho \lambda \alpha v \delta i ́ \alpha$ & 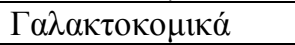 & 2.110 \\
\hline 15. & SOCOPA & $\Gamma \alpha \lambda \lambda i ́ \alpha$ & 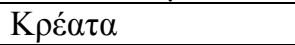 & 1.930 \\
\hline 16. & Flora Holland & O$\lambda \lambda \alpha \nu \delta \delta i \alpha$ & 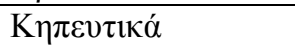 & 1.919 \\
\hline 17. & DLG & $\Delta \alpha v i ́ \alpha$ & E $\varphi$ ó $\delta 1 \alpha$ & 1.880 \\
\hline 18. & Sodiaal & $\Gamma \alpha \lambda \lambda i ́ \alpha$ & 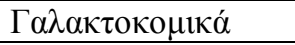 & 1.870 \\
\hline 19. & Irish Dairy Board & I $\rho \lambda \alpha v \delta i ́ \alpha$ & 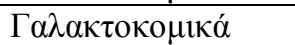 & 1.791 \\
\hline 20. & TEREOS & $\Gamma \alpha \lambda \lambda i \alpha$ & 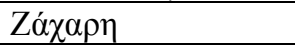 & 1.729 \\
\hline 21. & Valio group & $\Phi ı v \lambda \alpha v \delta i ́ \alpha$ & 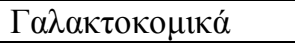 & 1.600 \\
\hline 22. & Bloemenveiling & O $\lambda \lambda \alpha v \delta i ́ \alpha$ & 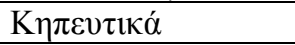 & 1.598 \\
\hline 23. & The Greenery & O $\lambda \lambda \alpha v \delta i ́ \alpha$ & 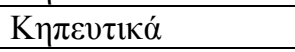 & 1.570 \\
\hline 24. & RWZ Rhein-Main & 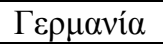 & 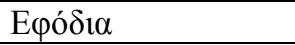 & 1.543 \\
\hline 25. & RWA & 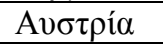 & 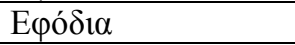 & 1.530 \\
\hline 26. & Sodra & 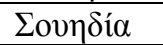 & 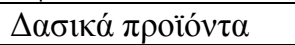 & 1.400 \\
\hline 27. & Bretagne & $\Gamma \alpha \lambda \lambda i ́ \alpha$ & 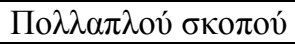 & 1.370 \\
\hline 28. & CECAB & $\Gamma \alpha \lambda \lambda i \alpha$ & 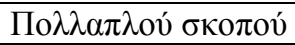 & 1.348 \\
\hline 29. & AGRIAL & $\Gamma \alpha \lambda \lambda i ́ \alpha$ & 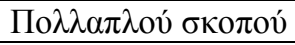 & 1.328 \\
\hline 30. & Cosun-Breda & O$\lambda \lambda \alpha \alpha v \delta i ́ \alpha$ & 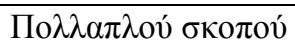 & 1.321 \\
\hline
\end{tabular}

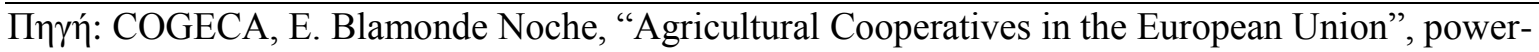
point presentation, Madrid, 22.11.2005

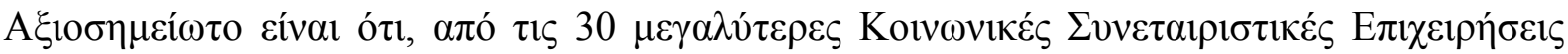

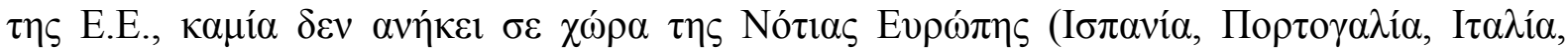

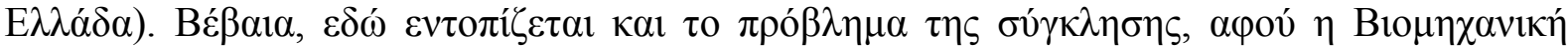

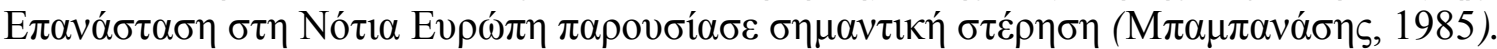

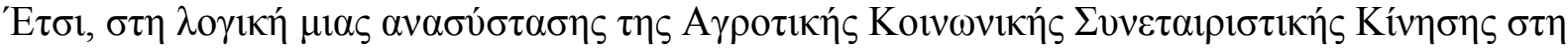

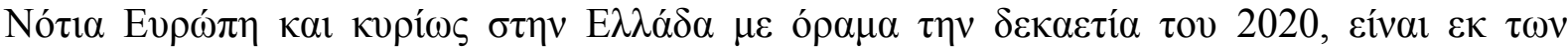

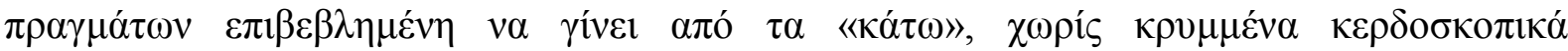

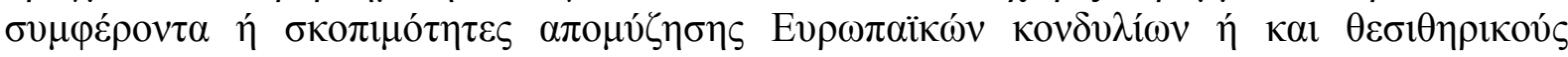

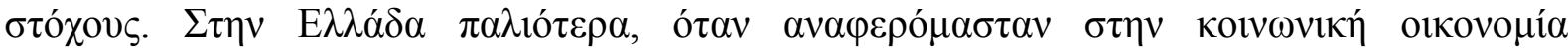

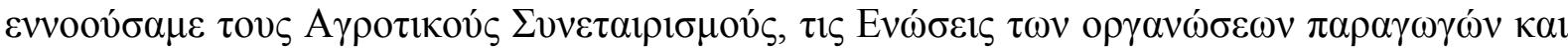

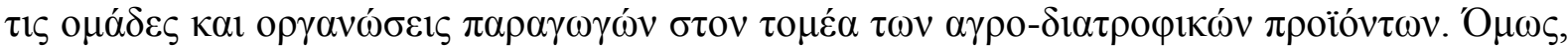

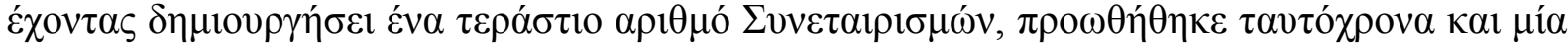

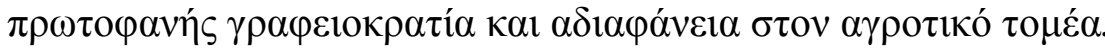

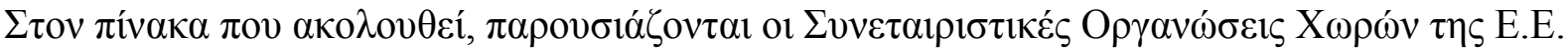
Пívakas 14

\begin{tabular}{|c|c|c|c|c|c|c|c|c|c|}
\hline 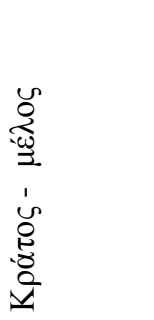 & 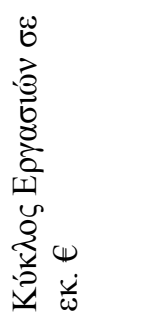 & 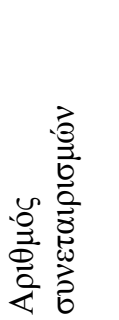 & 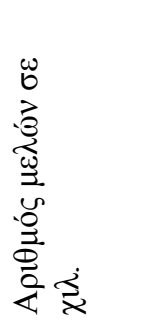 & 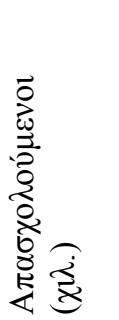 & 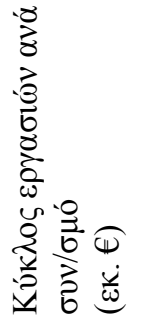 & 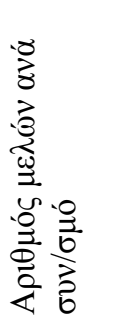 & 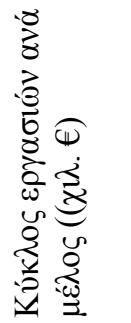 & 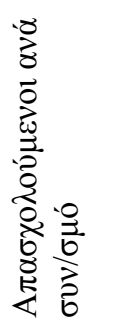 & 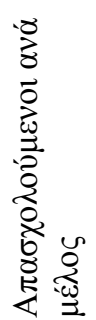 \\
\hline 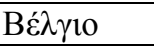 & 2750 & 355 & 35,8 & 20,0 & 7,75 & 101 & 76,82 & 56,3 & 0,6 \\
\hline$\Delta \alpha v^{\prime} \alpha$ & 18850 & 14 & 81,5 & 35,0 & 1346,43 & 5821 & 231,29 & 2500,0 & 0,4 \\
\hline 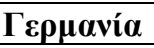 & 37000 & 3286 & 2385,0 & 120,0 & 11,26 & 726 & 15,51 & 36,5 & $\mathbf{0 , 1}$ \\
\hline$E \lambda \lambda \alpha \alpha^{\prime} \delta \alpha$ & 1040 & 6370 & 714,0 & - & 0,16 & 112 & 1,46 & - & - \\
\hline 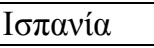 & 14190 & 4175 & 932,1 & 78,4 & 3,40 & 223 & 15,22 & 18,8 & 0,1 \\
\hline$\Phi ı v \lambda \alpha v \delta i ́ \alpha$ & 13300 & 48 & 215,0 & 45,2 & 277,08 & 4479 & 61,86 & 941,7 & 0,2 \\
\hline$\Gamma \alpha \lambda \lambda i{ }^{\prime} \alpha$ & 67000 & 3500 & 580,0 & 150,0 & 19,14 & 166 & 115,52 & 42,9 & 0,3 \\
\hline I $\rho \lambda \alpha v \delta i ́ \alpha$ & 12400 & 99 & 197,9 & 35,3 & 125,25 & 1999 & 62,66 & 356,5 & 0,2 \\
\hline$I \tau \alpha \lambda i ́ \alpha$ & 27070 & 5164 & 783,8 & 86,1 & 5,24 & 152 & 34,54 & 16,7 & 0,1 \\
\hline O $\lambda \lambda \alpha v \delta i ́ \alpha$ & 45160 & 44 & 143,1 & 59,6 & 1026,36 & 3253 & 315,50 & 1354,5 & 0,4 \\
\hline
\end{tabular}




\begin{tabular}{|c|c|c|c|c|c|c|c|c|c|}
\hline 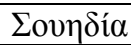 & 12600 & 34 & 300,0 & 30,0 & 370,59 & 8824 & 42,00 & 882,4 & 0,1 \\
\hline$\Sigma u ́ v o \lambda o$ & 251360 & 23089 & 6368,3 & 659,6 & 10,89 & 276 & 39,47 & 28,6 & 0,1 \\
\hline
\end{tabular}

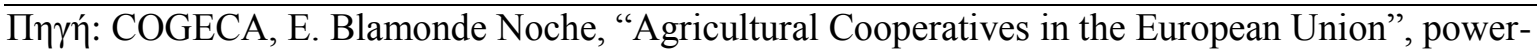
point presentation, Madrid, 22.11.2005

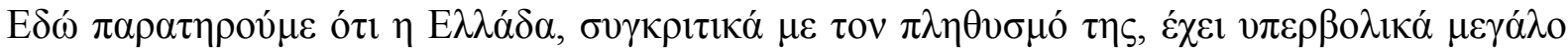

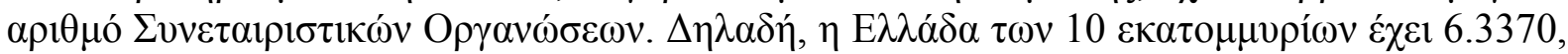

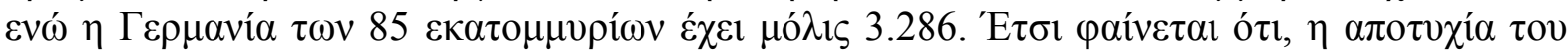

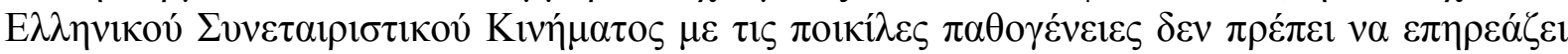

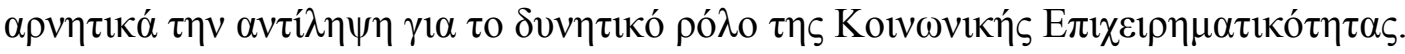

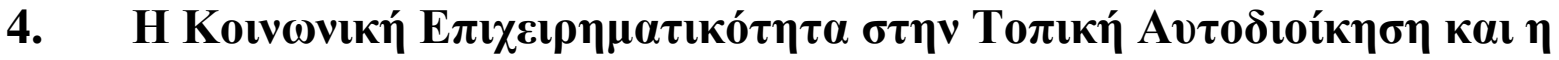

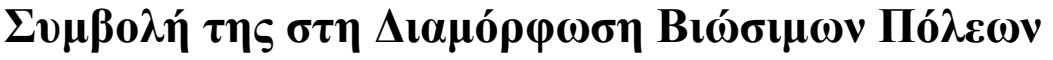

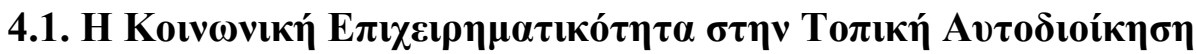

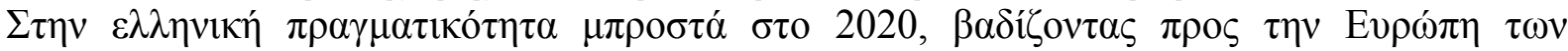

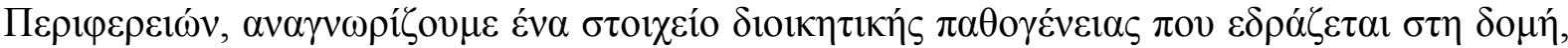

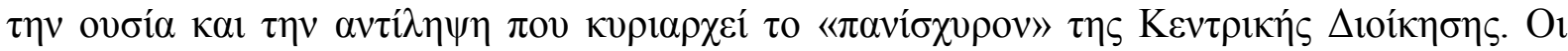

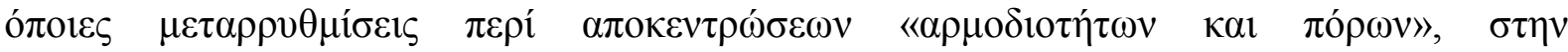

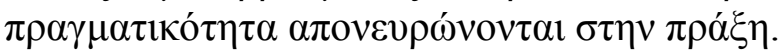

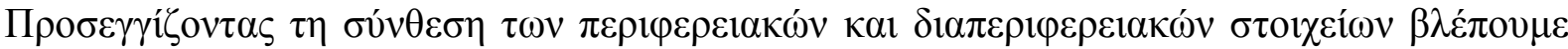

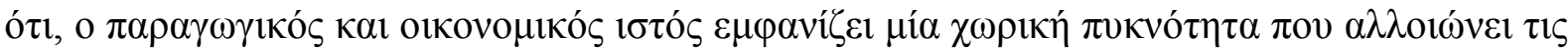

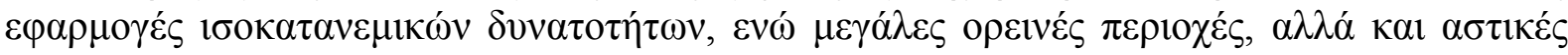

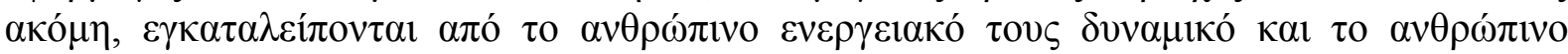

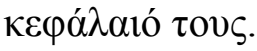

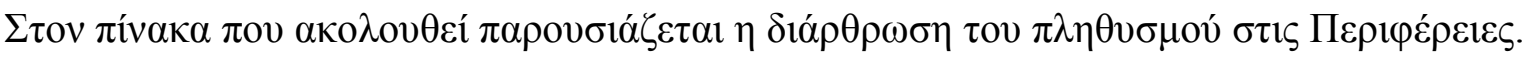

Пívaкas 15

\begin{tabular}{|c|c|c|c|c|}
\hline ПЕРІФЕРЕІЕ $\Sigma$ & $\begin{array}{c}\text { ¿YMMETOXH } \\
\%\end{array}$ & 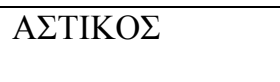 & АГРОТІКОГ & HMIA 2 TIKO $\Sigma$ \\
\hline 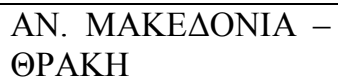 & & 40 & 43 & 17 \\
\hline $\begin{array}{l}\text { KENTPIKH } \\
\text { MAKE } \Delta \text { ONIA }\end{array}$ & 17,1 & 57 & 26 & 17 \\
\hline $\begin{array}{l}\text { SYTIKH } \\
\text { MAKE } \Delta \text { ONIA }\end{array}$ & & 29 & 56 & 15 \\
\hline$\Theta \mathrm{E} \Sigma \Sigma \mathrm{A} \Lambda \mathrm{IA}$ & 7,1 & 44 & 40 & 14 \\
\hline HПЕIPO $\Sigma$ & 3,5 & 31 & 59 & 10 \\
\hline ATTIKH & 37,8 & 94 & 1 & 5 \\
\hline ПЕ $\Lambda$ OПONNH $\Sigma O \Sigma$ & 6,4 & 24 & 57 & 19 \\
\hline$\Sigma$ TEPEA E $\Lambda \Lambda \mathrm{A} \triangle \mathrm{A}$ & 6,3 & 25 & 45 & 30 \\
\hline KPHTH & 5,3 & 42 & 46 & 12 \\
\hline BOPEIO АIГAIO & 1,7 & 28 & 55,5 & 17 \\
\hline NOTIO AIГAIO & 2,6 & 35 & 40 & 25 \\
\hline IONIA NH $\Sigma$ IA & 1,9 & 26 & 63 & 11 \\
\hline$\Delta$ YTIKH E $\Lambda \Lambda \mathrm{A} \Delta \mathrm{A}$ & & 45 & 43,5 & 11,5 \\
\hline ME $\Sigma \mathrm{O} \Sigma \mathrm{X} \Omega \mathrm{PA} \Sigma$ & & 40,6 & 44,2 & 15,6 \\
\hline
\end{tabular}

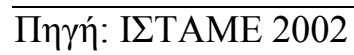




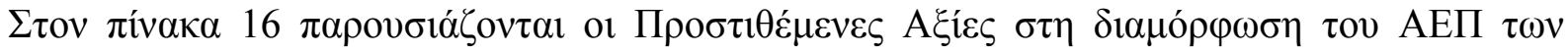

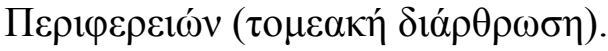

Пívakas 16:

\begin{tabular}{|c|c|c|c|c|}
\hline ПЕРІФЕРЕІЕ $\Sigma$ & $\begin{array}{l}\text { इYMMETOXH } \\
\Sigma \text { TO AEП (\%) }\end{array}$ & ПРЛТОГЕNН $\Sigma$ & $\triangle$ ЕYТЕРОГЕNН $\Sigma$ & ТРІТОГЕNН $\Sigma$ \\
\hline $\begin{array}{l}\text { AN. MAKE } \triangle \text { ONIA - } \\
\text { @PAKH }\end{array}$ & 5,30 & 25,00 & 36,00 & 39,00 \\
\hline $\begin{array}{l}\text { KENTPIKH } \\
\text { MAKE } \triangle \text { ONIA }\end{array}$ & 17,00 & 18,00 & 28,00 & 54,00 \\
\hline $\begin{array}{l}\text { YTIKH } \\
\text { MAKE } \Delta \text { ONIA }\end{array}$ & 2,60 & 13,40 & 47,50 & 39,10 \\
\hline$\Theta \mathrm{E} \Sigma \Sigma \mathrm{A} \Lambda \mathrm{IA}$ & 6,10 & 35,50 & 22,40 & 43,10 \\
\hline HПЕIPO $\Sigma$ & 2,70 & 17,00 & 23,00 & 60,00 \\
\hline ATTIKH & 38,00 & 2,20 & 27,90 & 72,90 \\
\hline ПЕ $\Lambda$ OПONNH $\Sigma O \Sigma \Sigma$ & 4,80 & 30,50 & 23,20 & 46,30 \\
\hline$\Sigma$ TEPEA Е $\Lambda \Lambda \mathrm{A} \triangle \mathrm{A}$ & 5,80 & 21,90 & 41,90 & 36,20 \\
\hline KPHTH & 5,70 & 31,00 & 13,00 & 56,00 \\
\hline ВОРЕIO АIГАIО & 1,60 & 20,00 & 17,00 & 63,00 \\
\hline NOTIO AIГAIO & 3,20 & 10,00 & 16,00 & 74,00 \\
\hline IONIA NH $\Sigma$ IA & 1,70 & 17,00 & 14,00 & 69,00 \\
\hline$\Delta$ YTIKH E $\Lambda \Lambda \mathrm{A} \Delta \mathrm{A}$ & 5,90 & 27,00 & 21,00 & 51,00 \\
\hline
\end{tabular}

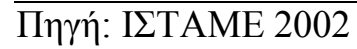

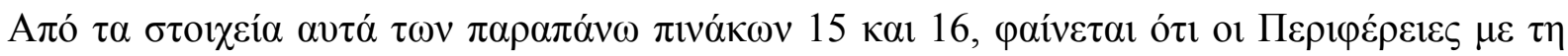

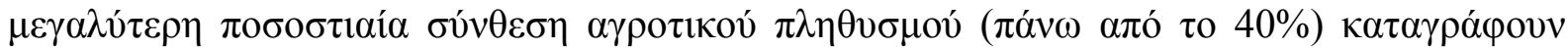

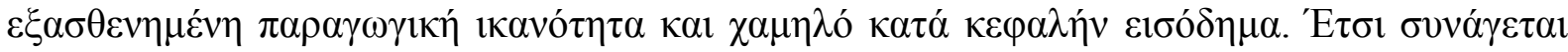

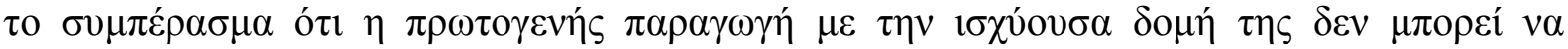

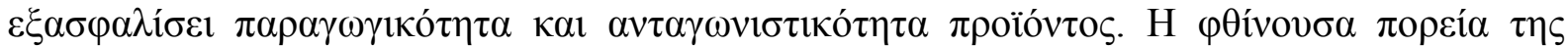

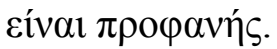

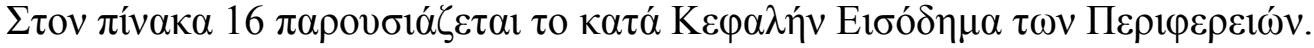

Пívakas 17:

\begin{tabular}{|c|c|c|}
\hline ПЕРІФЕРЕІЕ $\Sigma$ & $\triangle$ EKAETIA $1990(\%)$ & \\
\hline AN. MAKE $\triangle$ ONIA - $\Theta P A K H$ & 85,90 & 85,5 \\
\hline KENTPIKH MAKE $\triangle$ ONIA & 94,10 & 93,0 \\
\hline$\Delta$ YTIKH MAKE $\Delta$ ONIA & 88,60 & 76,00 \\
\hline 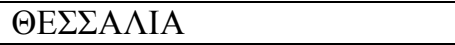 & 88,70 & 99,50 \\
\hline HПЕIPO $\Sigma$ & 69,20 & 63,50 \\
\hline ATTIKH & 109,00 & 109,00 \\
\hline 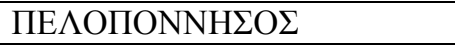 & 93,10 & 84,90 \\
\hline$\Sigma$ TEPEA E $\Lambda \Lambda \mathrm{A} \triangle \mathrm{A}$ & 112,30 & 87,60 \\
\hline KPHTH & 106,50 & 103,70 \\
\hline ВОРЕIO АІГАIО & 67,30 & 72,30 \\
\hline NOTIO AIГAIO & 108,20 & 106,80 \\
\hline IONIA NH $\Sigma$ IA & 82,50 & 82,50 \\
\hline$\Delta$ YTIKH E $\Lambda \Lambda \mathrm{A} \Delta \mathrm{A}$ & 80,30 & 82,60 \\
\hline
\end{tabular}

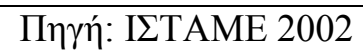

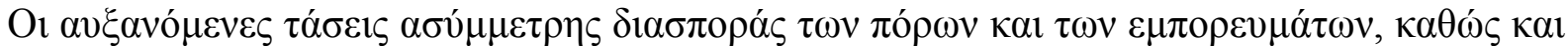

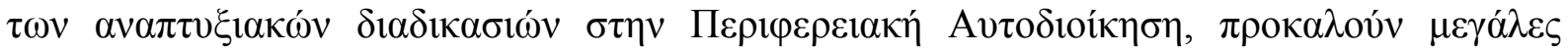




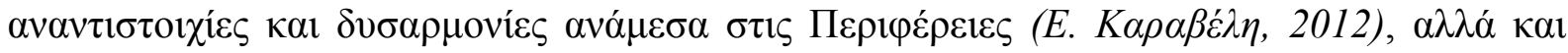

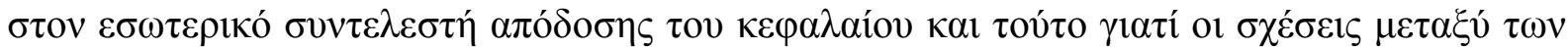

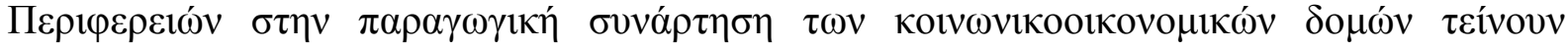

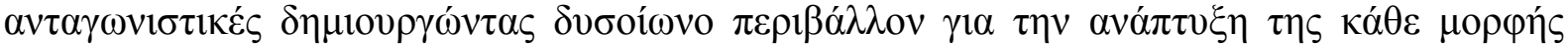

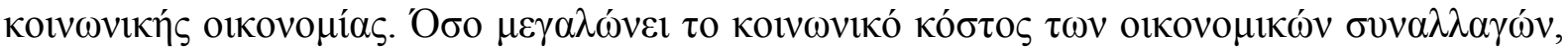

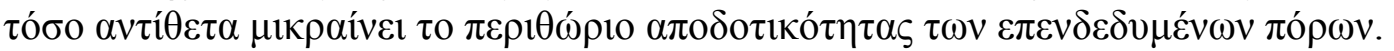

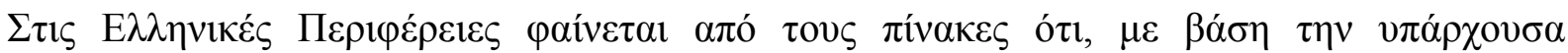

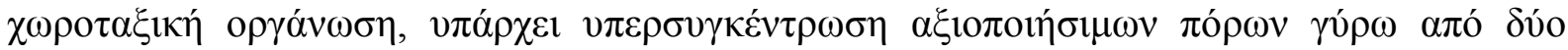

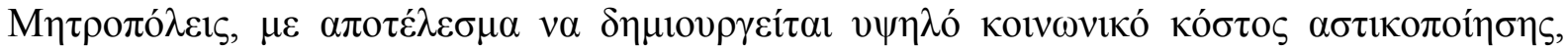

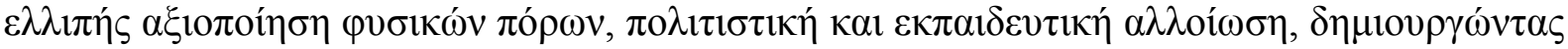

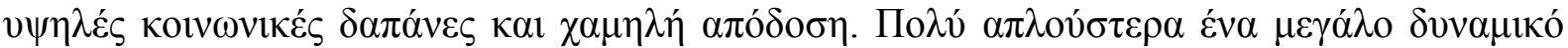

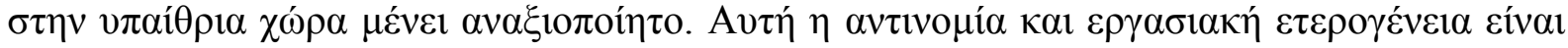

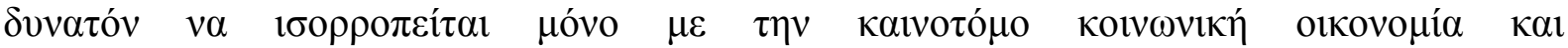

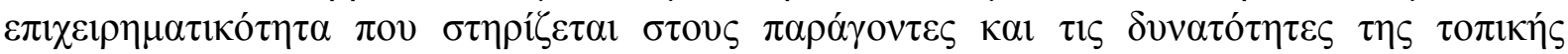

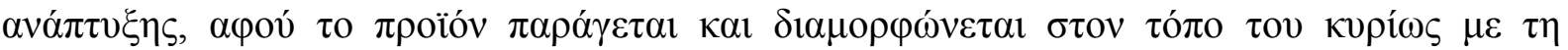

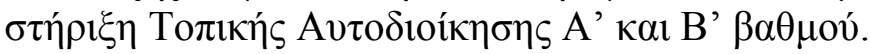

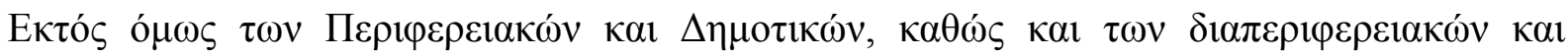

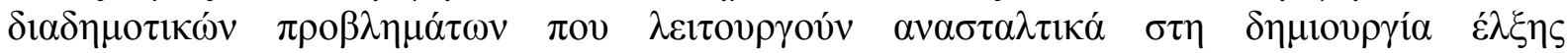

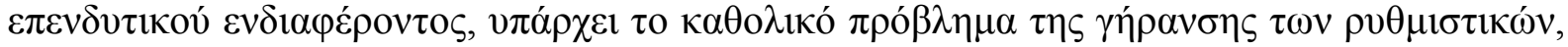

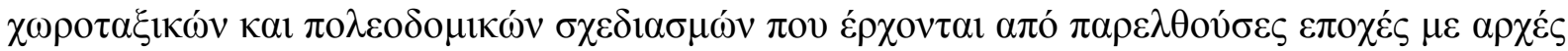

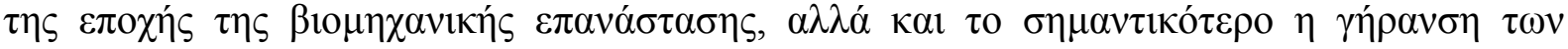

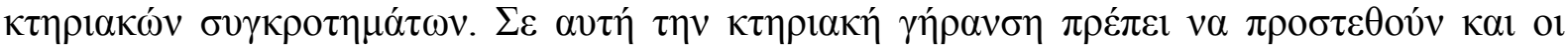

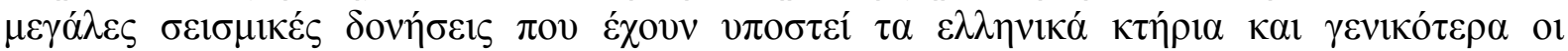

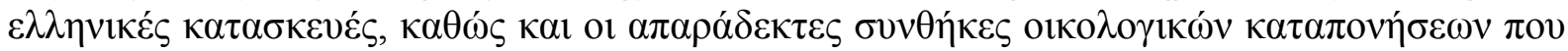

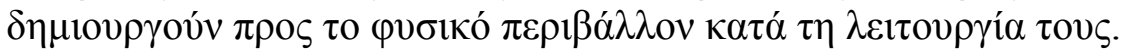

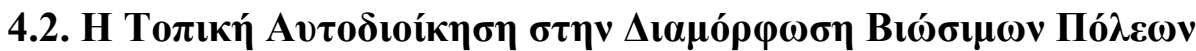

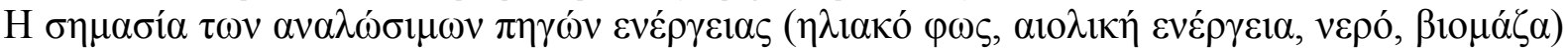

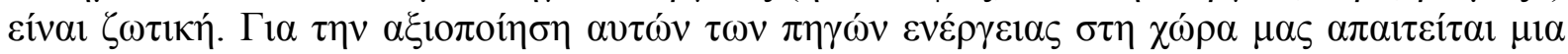

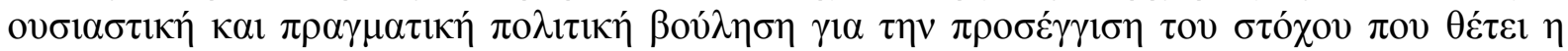

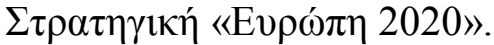

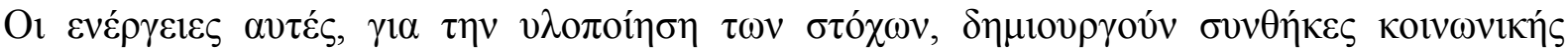

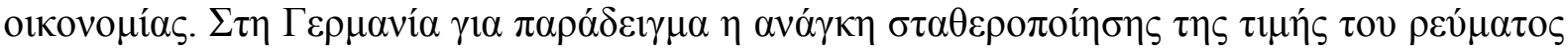

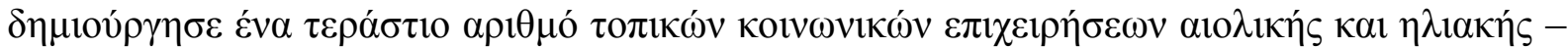

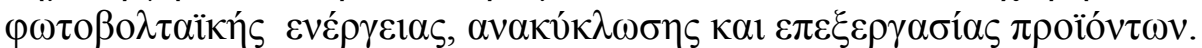

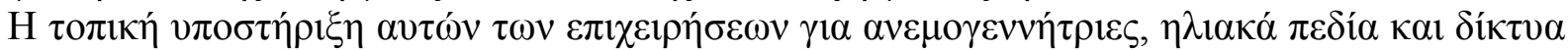

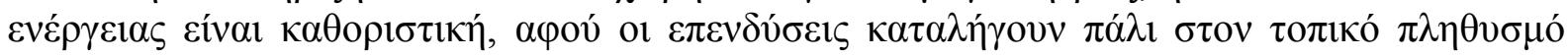

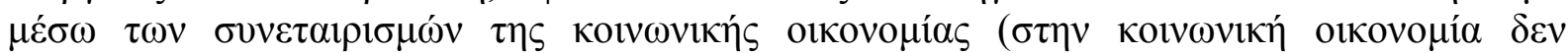

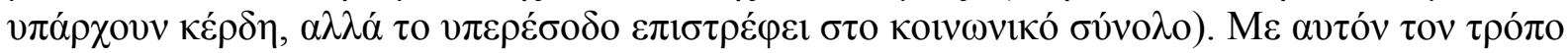

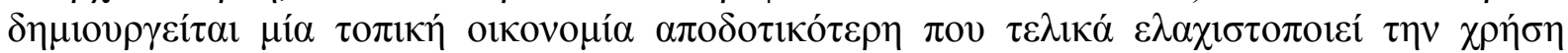

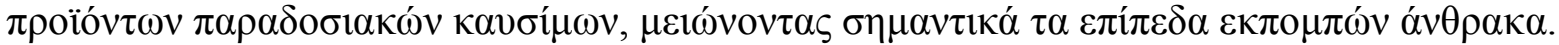

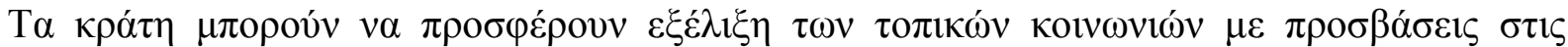

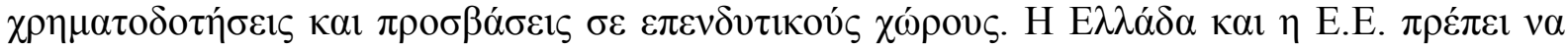

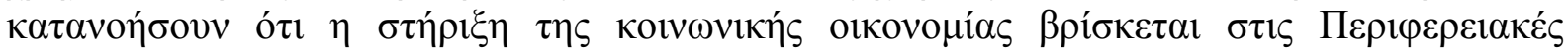

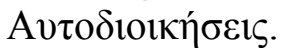

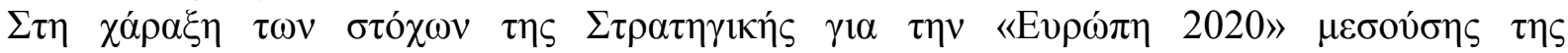

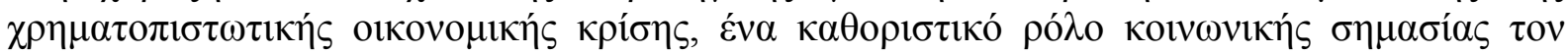

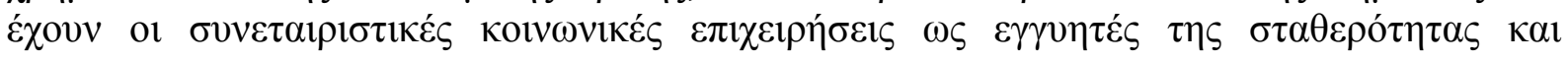




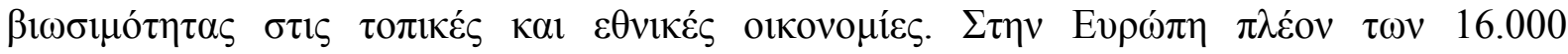

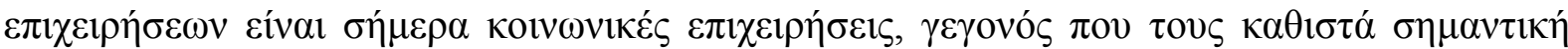

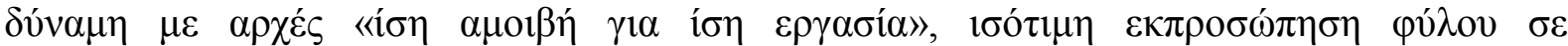

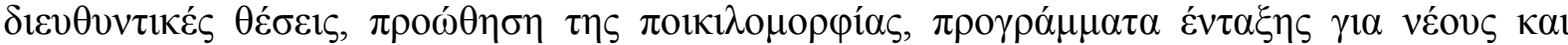

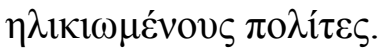

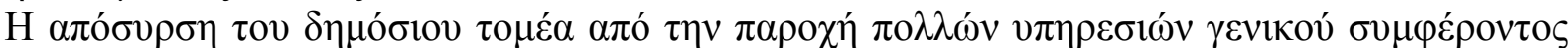

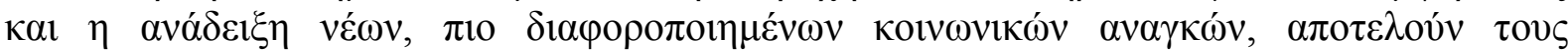

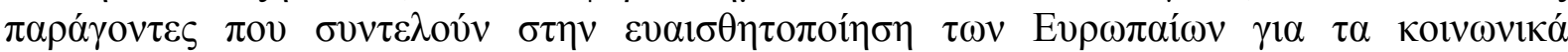
$\pi \rho \beta \beta \lambda \eta \dot{\mu} \mu \tau \alpha$.

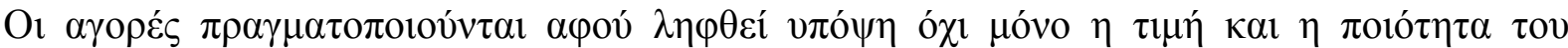

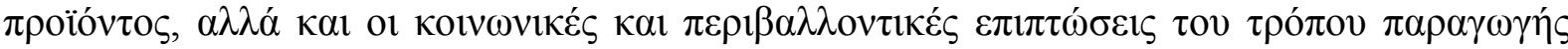

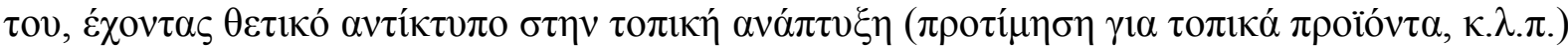

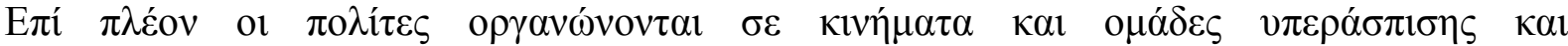

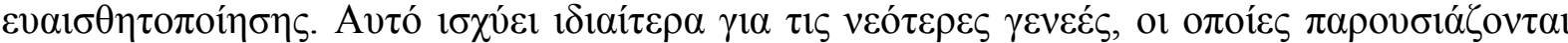

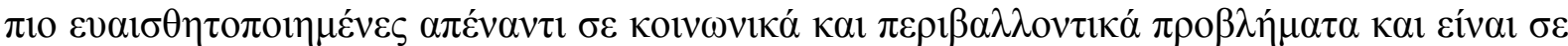

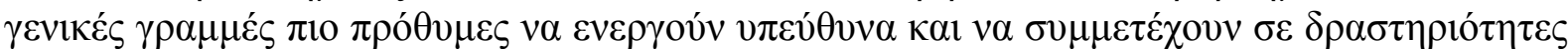

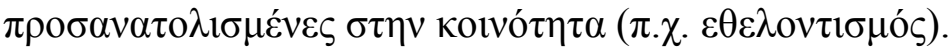

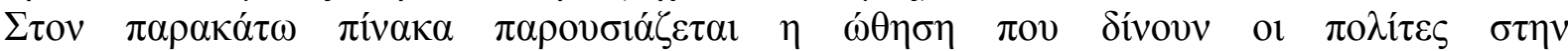

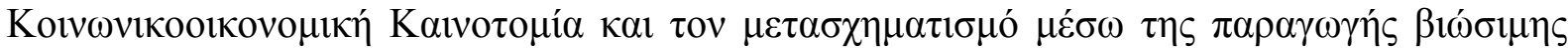

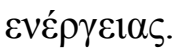

Пívakas 18

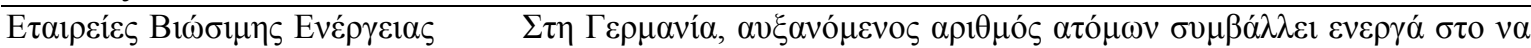

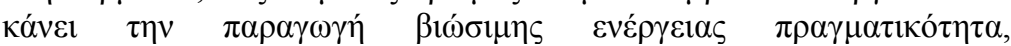

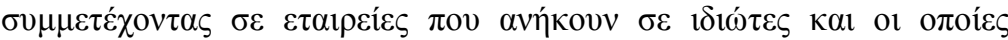

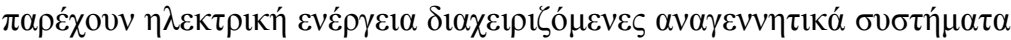

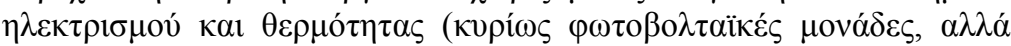

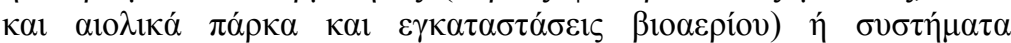

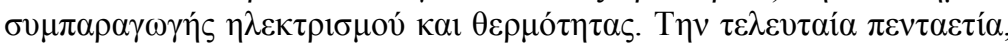

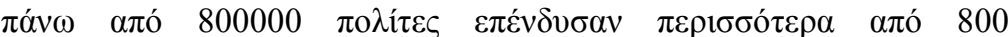

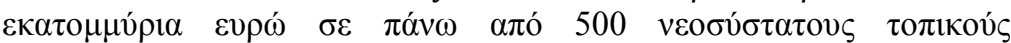

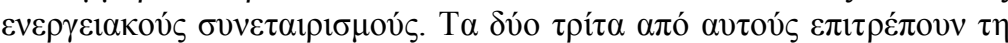

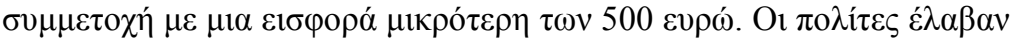

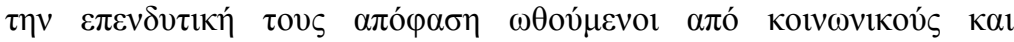

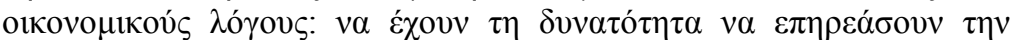

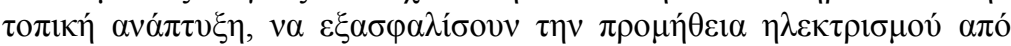

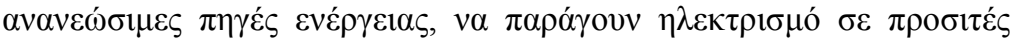

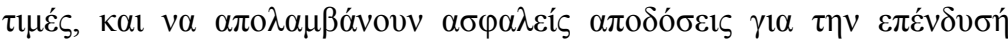

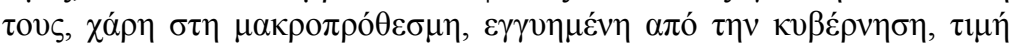

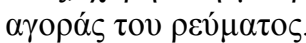

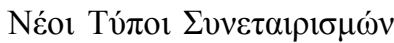

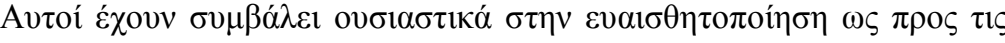

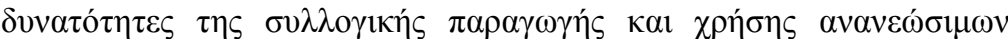

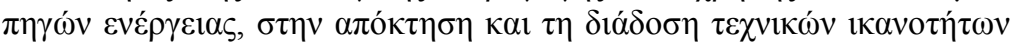

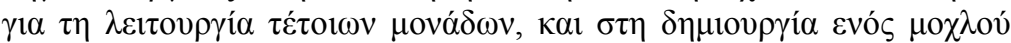

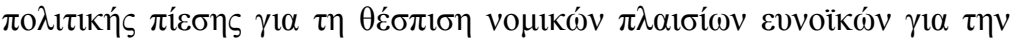

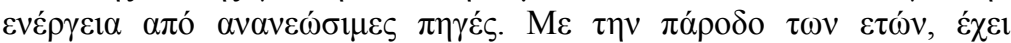

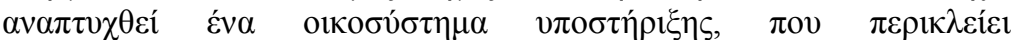

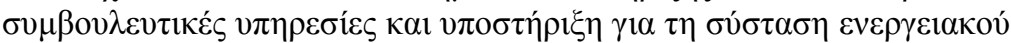

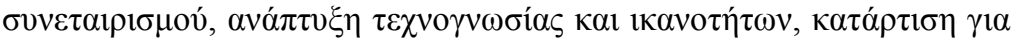

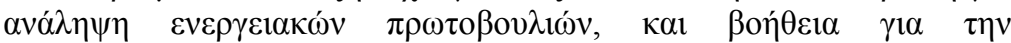
$\alpha v \alpha \pi \alpha \rho \alpha \gamma \omega \gamma \eta \dot{~ \kappa \alpha 1 ~ \pi \rho о \sigma \alpha \rho \mu о \gamma \eta ́ ~ \delta о к ц \mu \alpha \sigma \mu \varepsilon ́ v \omega v ~ \pi \rho о \tau и ́ \pi \omega v . ~}$

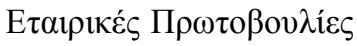

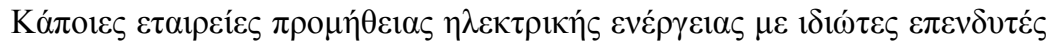

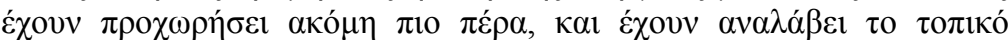

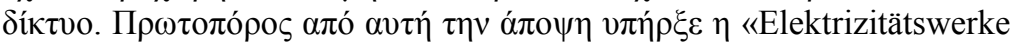

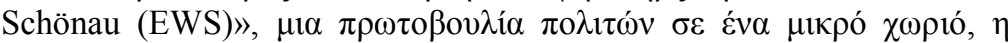

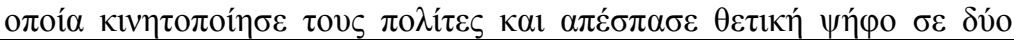




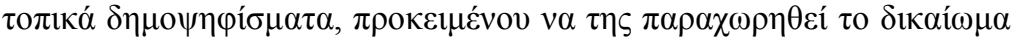

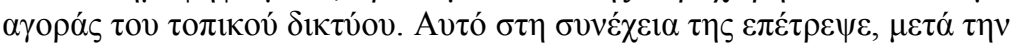

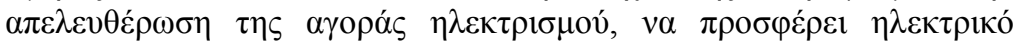

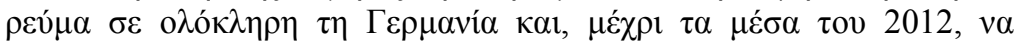

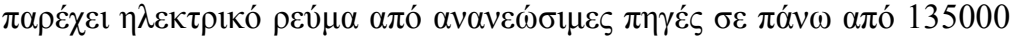

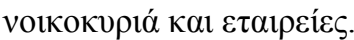

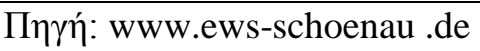

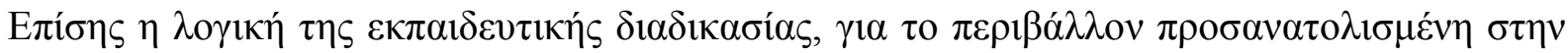

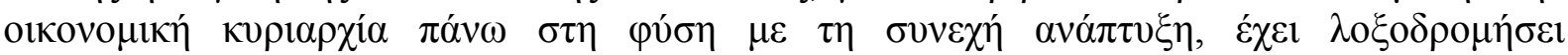

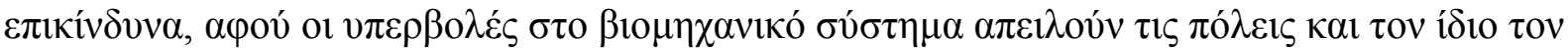

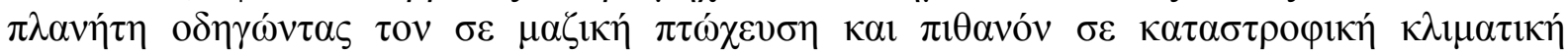

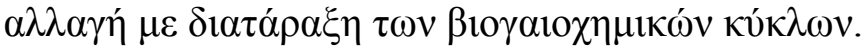

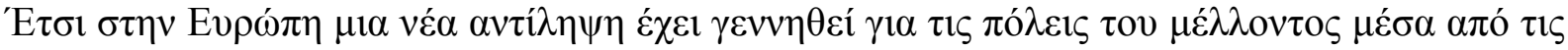

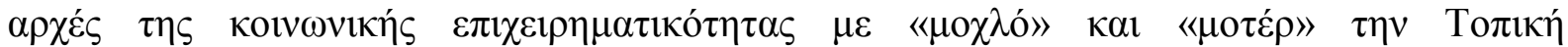

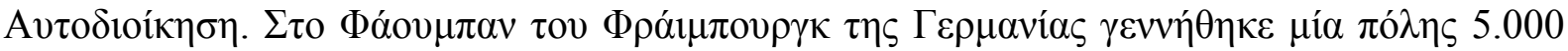

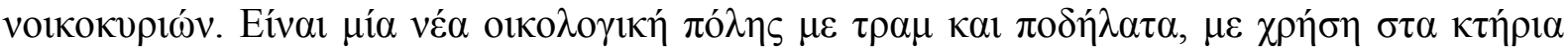

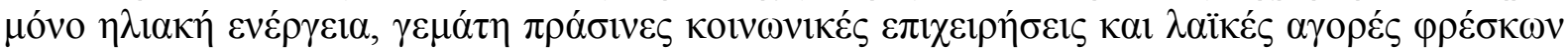

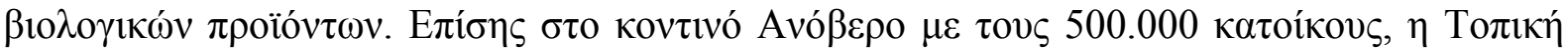

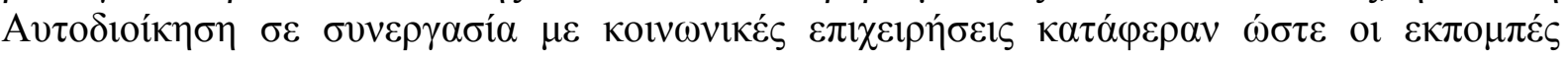

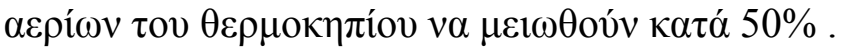

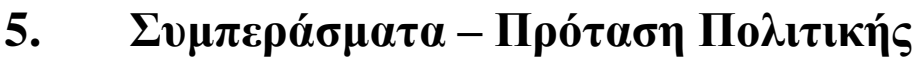

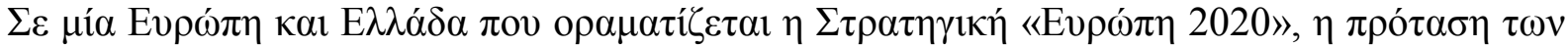

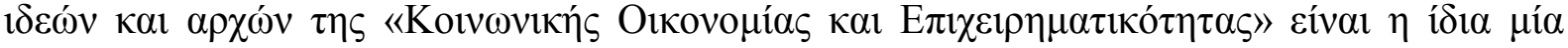

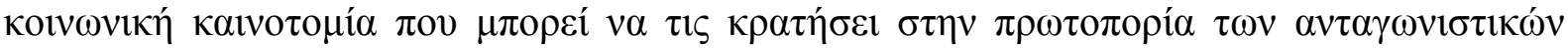

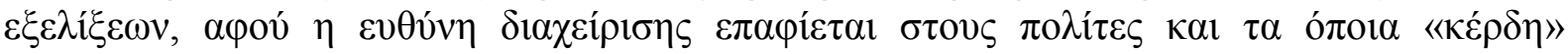

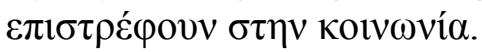

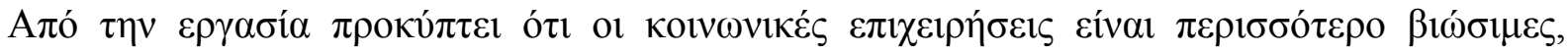

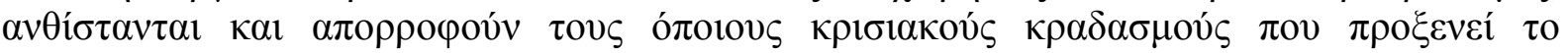

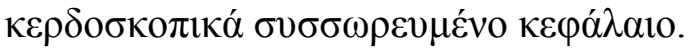

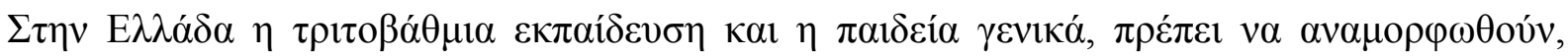

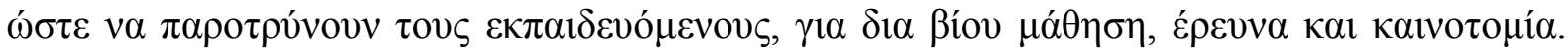

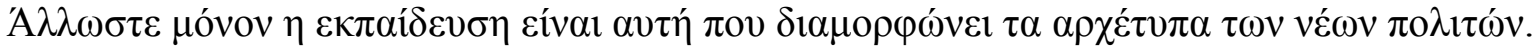

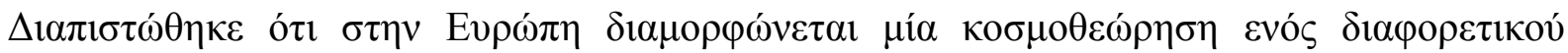

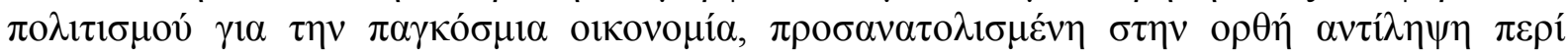

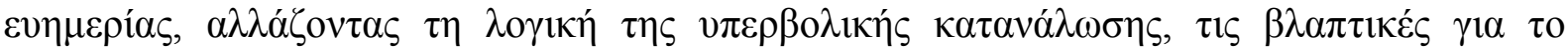

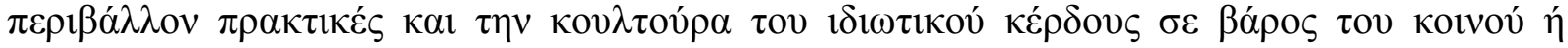

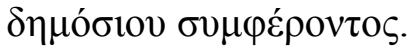

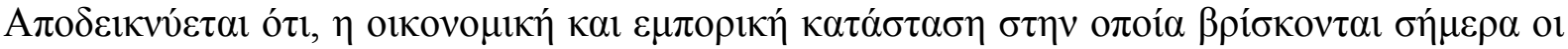

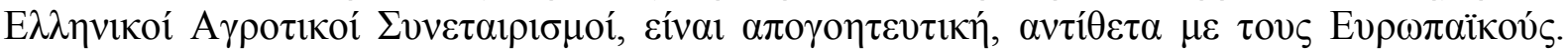

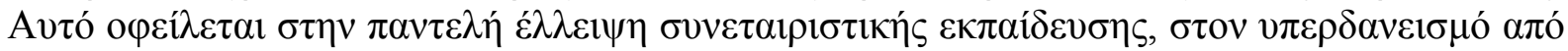

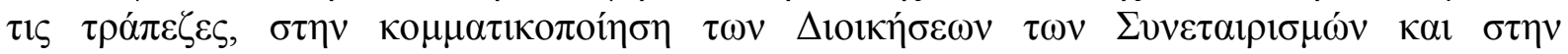

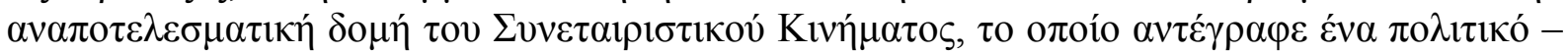

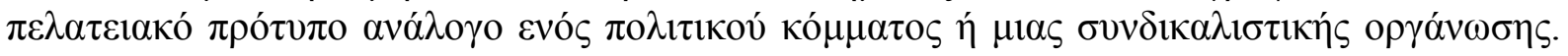




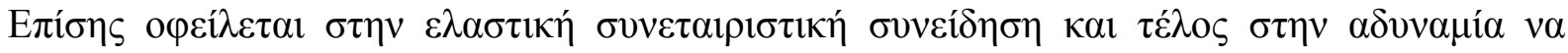

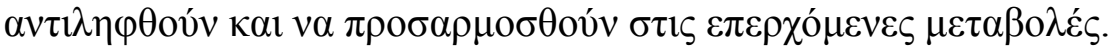

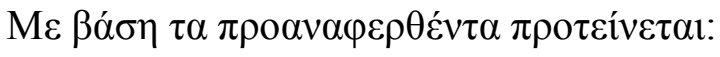

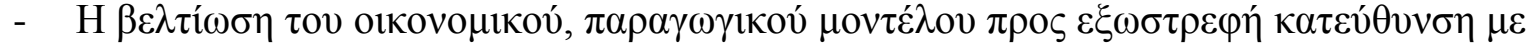

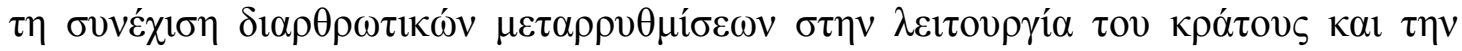

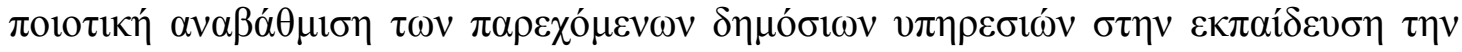

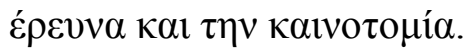

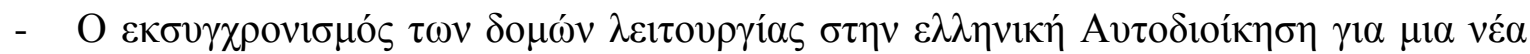

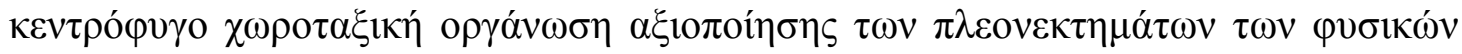
$\pi$ $\rho \omega v \tau \eta \varsigma \mathrm{E} \lambda \lambda \alpha \dot{\delta} \delta \alpha \varsigma$.

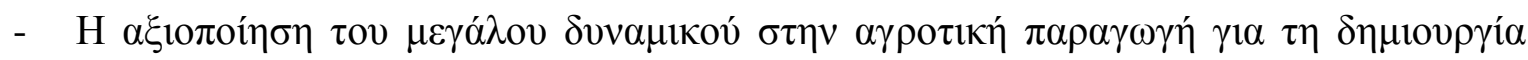

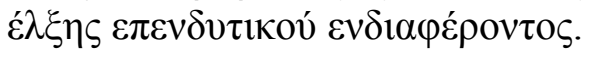

- Н

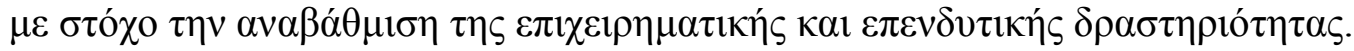

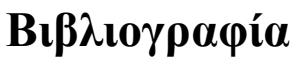

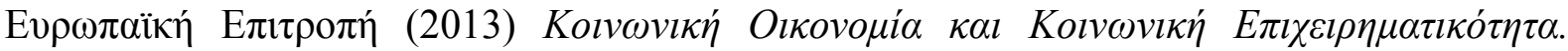

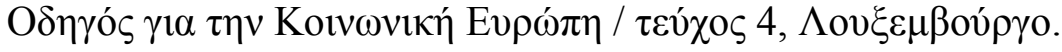

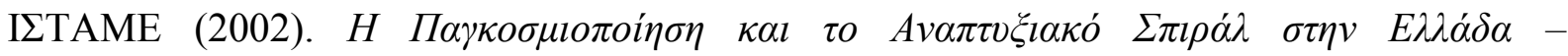

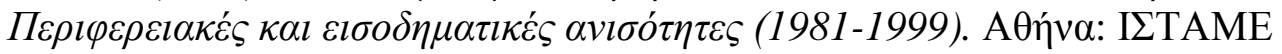

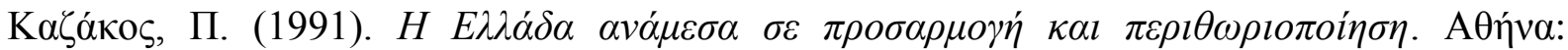

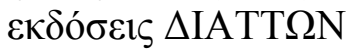

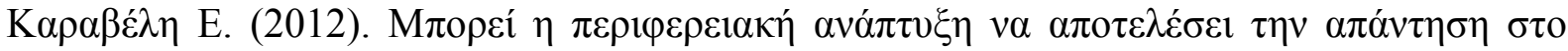

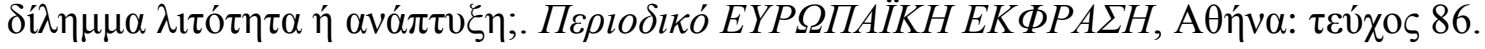

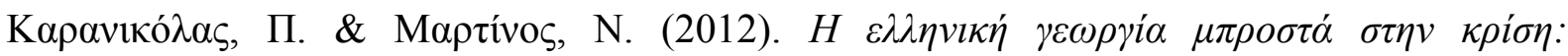

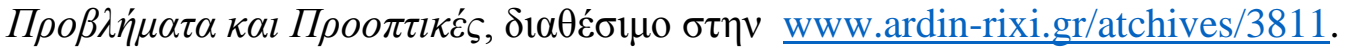

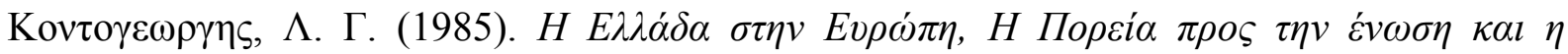

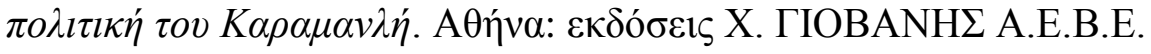

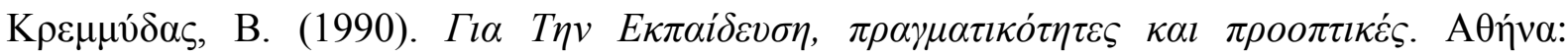
$\varepsilon \kappa \delta o ́ \sigma \varepsilon 1 \zeta \Gamma \mathrm{N} \Omega \Sigma \mathrm{H}$.

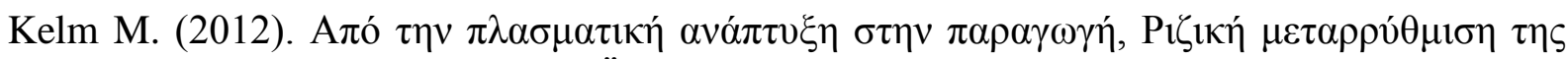

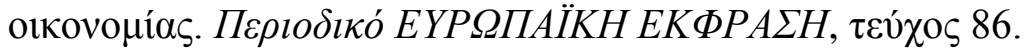

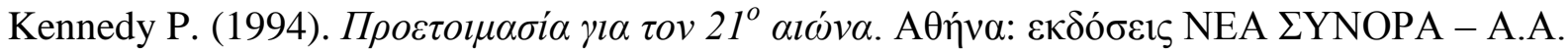
$\Lambda$ IBANH.

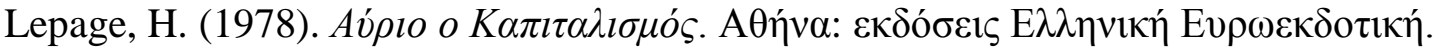

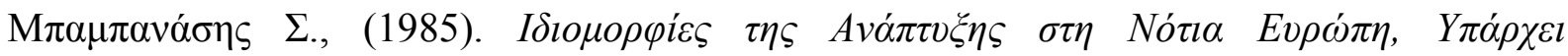

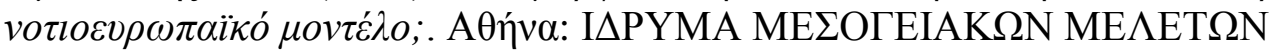

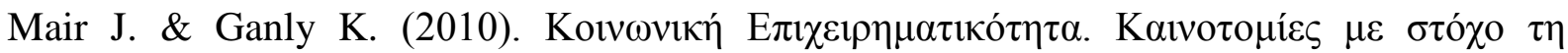

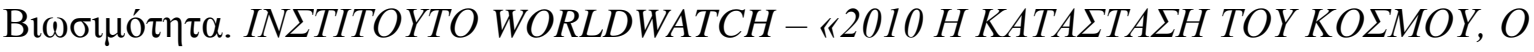




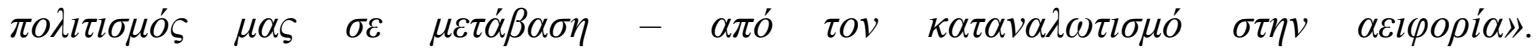
A $\theta \dot{v} \alpha: E U R O B A N K$.

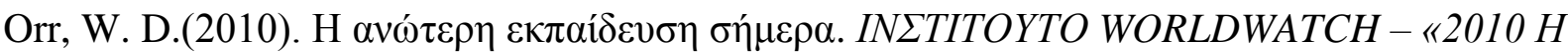

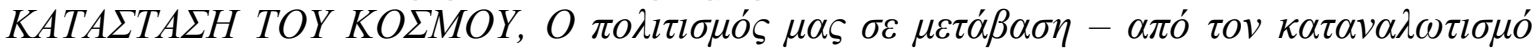

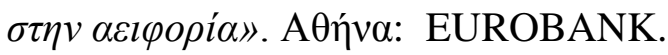

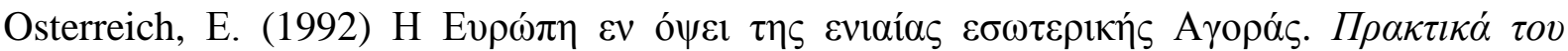

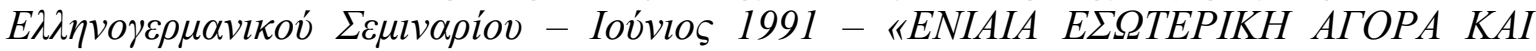

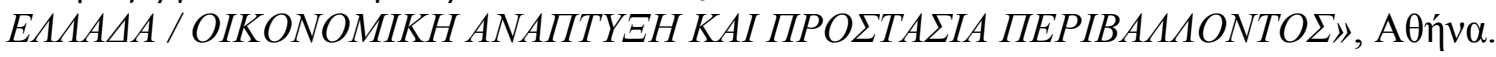

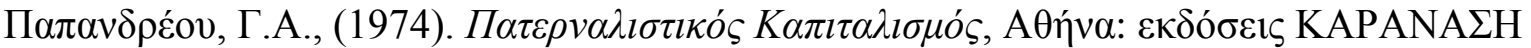

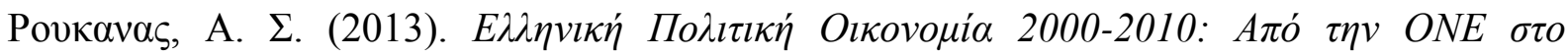

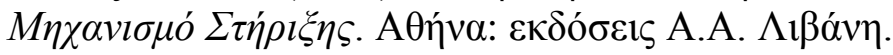

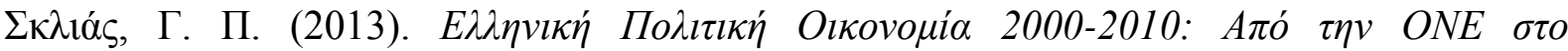

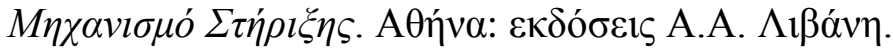

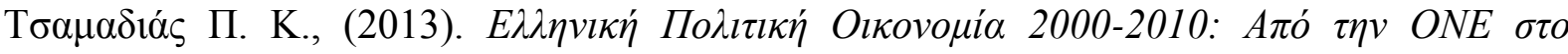

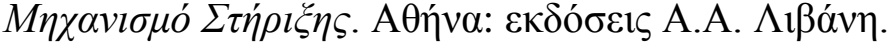

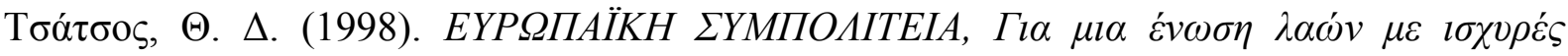

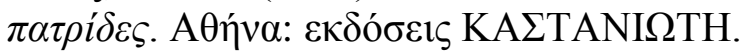

